\title{
Earthquake recordings from the 2002 Seattle Seismic Hazard Investigation of Puget Sound (SHIPS), Washington State
}

Thomas L. Pratt ${ }^{1}$, Karen L. Meagher ${ }^{2}$, Thomas M. Brocher ${ }^{3}$, Thomas Yelin ${ }^{2}$, Robert Norris $^{2}$, Lynn Hultgrien ${ }^{2}$, Elizabeth Barnett ${ }^{2}$, Craig S. Weaver ${ }^{2}$

Open-File Report 03-361

2003

This report is preliminary and has not been reviewed for conformity with U.S. Geological Survey editorial standards or with the North American Stratigraphic Code. Any use of trade, firm, or product names is for descriptive purposes only and does not imply endorsement by the U.S. Government.

U.S. Department of the Interior

U.S. Geological Survey

${ }^{1}$ U.S. Geological Survey, School of Oceanography, Box 357940, Univ. of Washington, Seattle, WA 98195

${ }^{2}$ U.S. Geological Survey, Earth and Space Sciences, Box 351650, Univ. of Washington, Seattle, WA 98195

${ }^{3}$ U.S. Geological Survey, 345 Middlefield Road, M/S 977, Menlo Park, CA 94025 
TABLE OF CONTENTS



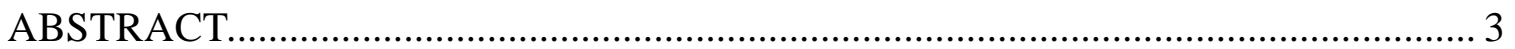

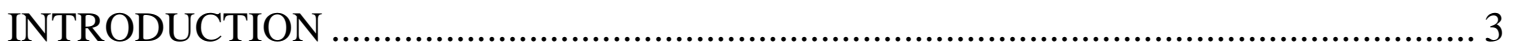

EXPERIMENT DESIGN ..................................................................................... 4

SEISMOGRAPH DEPLOYMENT AND MAINTENANCE …..................................... 5

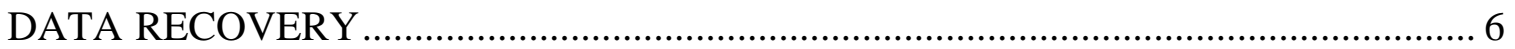

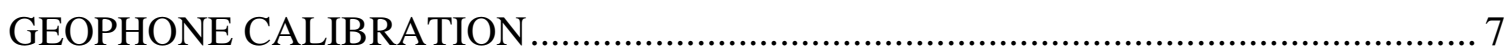

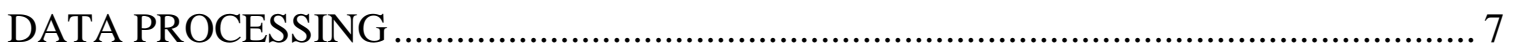

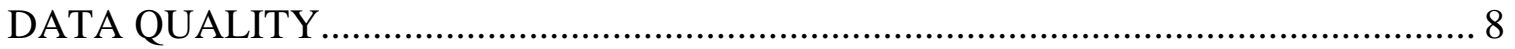

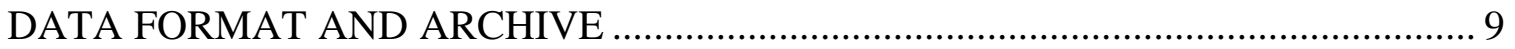

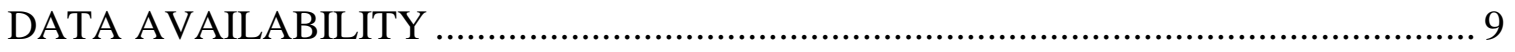

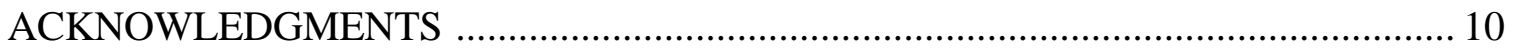



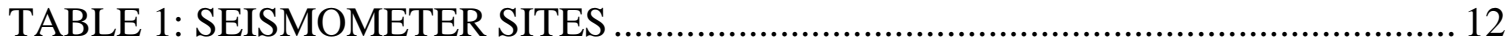

TABLE 2: GEOPHONE CALIBRATION RESULTS …............................................. 15

TABLE 3: LOCAL EARTHQUAKES RECORDED ON THE SEATTLE SHIPS

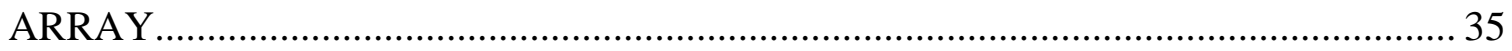

TABLE 4: LOCAL BLASTS RECORDED ON THE SEATTLE SHIPS ARRAY ........ 37

TABLE 5: TELESEISMS RECORDED ON THE SEATTLE SHIPS ARRAY ............. 39

TABLE 6. PASSCAL SEGY TRACE HEADER FORMAT.......................................... 44

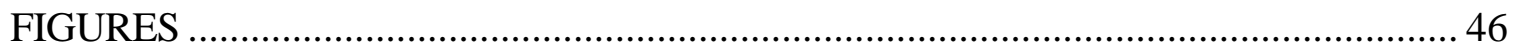

APPENDIX A: SITE VISITS, INSTRUMENT STATUS, AND DOWNLOADS .......... 66 


\section{ABSTRACT}

This report describes seismic data obtained during the fourth Seismic Hazard Investigation of Puget Sound (SHIPS) experiment, termed "Seattle SHIPS". The experiment was designed to study the influence of the Seattle sedimentary basin on ground shaking during earthquakes. To accomplish this, we deployed seismometers over the basin to record local earthquakes, quarry blasts, and teleseisms during the period of January 26 to May 27, 2002. We plan to analyze the recordings to compute spectral amplitudes at each site, to determine the variability of ground motions over the basin.

During the Seattle SHIPS experiment, seismometers were deployed at 87 sites in a 110-km-long east-west line, three north-south lines, and a grid throughout the Seattle urban area (Figure 1). At each of these sites, an $\mathrm{L}-22,2 \mathrm{~Hz}$ velocity transducer was installed and connected to a REF TEK Digital Acquisition System (DAS), both provided by the Program for Array Seismic Studies of the Continental Lithosphere (PASSCAL) of the Incorporated Research Institutes for Seismology (IRIS). The instruments were installed on January 26 and 27, and were retrieved gradually between April 18 and May 27. All instruments continuously sampled all three components of motion (velocity) at a sample rate of 50 samples/sec. To ensure accurate computations of amplitude, we calibrated the geophones in situ to obtain the instrument responses.

In this report, we discuss the acquisition of these data, we describe the processing and merging of these data into 1-hour long traces and into windowed events, we discuss the geophone calibration process and its results, and we display some of the earthquake recordings.

\section{INTRODUCTION}

The Seismic Hazard Investigations of Puget Sound (SHIPS) experiments are designed to better understand the earthquake hazard in the Puget Lowland region of Washington State (Pratt et al., 2002). During the 1998 "wet" SHIPS project, airgun shots behind the University of Washington's research vessel Thomas G. Thompson were recorded by a marine multichannel system and by 250 land-based seismic recorders (Fisher et al., 1999; Brocher et al., 1999). The 1999 "dry" SHIPS study was a refraction experiment in which 1008 land seismometers were placed along an east-west profile across the Seattle basin to record 38 underground detonations (Brocher et al., 2000a; Brocher et al., 2000b; Brocher et al., 2001b). The 2000 "Kingdome" SHIPS experiment used 207 land-based seismographs to record the destruction of the Kingdome sports stadium (equivalent to a M2.3 earthquake) and 4 underground explosions (Brocher et al., 2002; Brocher et al., 2000a).

The 2002 Seattle SHIPS experiment is predicated on the recordings of earthquakes during the first two SHIPS experiments. During the 1998 experiment, we analyzed recordings from 3 local earthquakes of magnitude 2.1 to 2.5 to look at ground shaking across the Puget Lowland, and the results demonstrated attenuation of highfrequency $(8$ to $20 \mathrm{~Hz}$ ) seismic waves by the Seattle basin (Pratt et al., 2003). Following the 1999 experiment, analyses of ground shaking during two local events, five blasts, and the M7.6 Chi-Chi, Taiwan, earthquake confirmed the high-frequency attenuation over the basin but demonstrated amplifications of 12 to 16 over the Seattle basin at low frequencies (0.1-1 Hz; Pratt et al., 2003; Brocher et al., 2000b). 
The results from these earlier SHIPS experiments, in which the analyses of ground motions were an afterthought, led to the planning of an experiment specifically to study the influence of the Seattle basin on ground shaking. The Seattle SHIPS experiment was designed to record ground motions during local events and teleseisms over a 3-month time period at a large number of sites distributed over and around the basin. The cancellation of an unrelated experiment allowed us to keep some of the instruments an extra month, until late May. This report describes the collection, processing and archiving of the data recorded during the 2002 Seattle SHIPS experiment.

\section{EXPERIMENT DESIGN}

The Seattle SHIPS experiment was designed to deploy and maintain a 3dimensional array of 87 similar seismographs distributed over the Seattle basin (Figures 1 and 2; Johnson a al., 1994; Pratt et al., 1997; Brocher et al., 2001a; VanWagoner et al., 2002). One set of 26 seismographs was deployed along an east-west line across the center of the basin and onto bedrock at both ends, approximately coincident with the 1999 SHIPS refraction profile (Brocher et al., 2000b; Snelson, 2001; Pratt et al., 2003). A north-south line of 24 instruments extended from Mukilteo (north of Seattle) to Federal Way (north of Tacoma), crossing the center of the Seattle basin and the areas of shallow bedrock (structural uplifts) that form the north and south edges of the basin. Two shorter north-south profiles, of seven stations each, crossed the basin east of Lake Washington and west of Puget Sound. Finally, a grid of receivers within the city of Seattle provided denser coverage over the urban area on the center of the basin.

We sited all but three of the instruments at private residences and businesses willing to let us draw power from their outdoor outlets. Volunteer property owners were first solicited by emailing our extensive database of people who had volunteered their houses during previous SHIPS experiments or who had volunteered to help in previous USGS projects, which resulted in about 180 potential sites. After plotting the volunteered sites on a map, we divided them into three categories: 1) primary sites that we wanted to use, 2) alternative sites near the primary sites, or 3) sites that were not in appropriate locations for our seismometers. We also identified additional locations where we wanted instruments but did not have any volunteered sites.

Once our potential seismometer locations were identified, USGS staff visited each site to confirm that the volunteered site (or a nearby alternative site) was suitable, or to knock on doors to find a homeowner or business owner willing to let us deploy a seismometer on their property. Requirements for each site were:

1) a relatively quiet location away from traffic, industrial operations, or other continuous sources of noise;

2) the ability to conceal the seismometer behind buildings or landscaping;

3) a convenient source of electrical power;

4) adequate sky view for the global positioning system (GPS) to work; and

5) accessibility for deploying and visiting the site 4 to 8 times over the 4-month period.

Three sites at the east end of the array were located on remote timberlands without electric power, so these instruments were powered by two 130 amp-hour, deepcycle batteries. 
Table 1 lists the final site locations by address, with latitude, longitude, UTM zone 10 Easting and Northing, elevation, instrument number and geophone number. The positions are determined from the average of the GPS locations recorded by the instruments, with elevations read from topographic maps. For each site, a sheet was prepared with driving directions, the owner's contact information, a site description, a listing of maintenance visits, and any special instructions. These sheets are included in this report as a Microsoft Excel spreadsheet on a CD written from a Microsoft Windows computer.

\section{SEISMOGRAPH DEPLOYMENT AND MAINTENANCE}

The seismographs were deployed on January 26 and 27 under adverse conditions - a snowstorm started on the afternoon of January 26 and continued through the next day. The 87 sites were grouped into 10 deployment routes of 7 to 11 instruments each, with teams of two people deploying each group of instruments. Teams with a large driving distance between sites were given fewer instruments to deploy. The same groupings of instruments used for deployment were also used to group instruments for maintenance.

At each site we deployed the following equipment (Figure 3):

1) a Mark products L-22, 2-Hz velocity transducer (geophone) buried 0.1 to $0.4 \mathrm{~m}$ deep, oriented with axes pointed to magnetic north and east;

2) a Refraction Technology (REF TEK) Digital Acquisition System (DAS);

3) a GPS clock with an external antenna (or an external clock) that could be extended to reach areas of clear sky;

4) a 70 amp-hour car battery to provide power to the DAS; and

5) a 0.5 -amp battery charger to maintain the charge on the battery.

All of the equipment except the seismometer and GPS antenna was placed inside a plastic case ("Action Packer" storage container) to keep it dry (Figure 3). At sites where the instrument had an open view of the sky, the GPS antenna also was placed inside the plastic case on top of the DAS. At most sites, the GPS antenna was mounted nearby where it had a good view of the sky.

Most (71) of the L-22 sensors were identical units provided by IRIS. The remaining 16 sensors were L-22s owned by the USGS. The USGS sensors had a variety of resistors in them to equalize the sensitivity, resulting in a uniformly lower sensitivity than the IRIS seismometers. The geophone characteristics (natural period, sensitivity), determined from in situ or lab calibration tests are described later. Geophones were buried to a depth of 0.1 to $0.4 \mathrm{~m}$ depending upon site conditions. Geophone axes were leveled with a bubble level and oriented with their axes pointed to magnetic north and east. In the Seattle area, magnetic north lies about $20^{\circ}$ east of true north.

Counting spares, we used 105 Digital Acquisition Systems (DASes) in the experiment, most (92) of which were REF TEK 07s with 0.5, 1.0, 2.0, or 4.0 gigabyte hard drives. These disk sizes allowed the stations to run continuously for 2 weeks $(0.5$ gigabyte drives on an 06 DAS) to 8 weeks (4 gigabyte drives on an 07 DAS). However, we visited the sites at least once every 4 weeks to check on the instruments and download data. Because we did not have enough 07 REF TEKs, thirteen of the instruments were REF TEK 06s. The 06 instruments have slightly smaller dynamic range because of smaller A/D size (24 versus 16 bits), and they had smaller disk sizes. The 06 instruments 
were used at noisier and more accessible sites within the city of Seattle, under the assumption that the dynamic range was less important because only the largest events would be recorded at these noisier sites. PASSCAL (1991) and Brocher et al. (1999) describe the REF TEK instruments. All instruments were programmed to record three channels continuously at 50 samples/sec during the duration of the experiment.

For maintenance, the array was divided into the same 10 routes used in the deployment, with five USGS staff each responsible for maintaining two of these routes. Appendix A lists the sites by numerical order within each of these deployment routes. Sites were visited every 2 to 4 weeks, depending upon the size of the disks. During each maintenance visit, the status of the instrument was checked and the data were downloaded to a laptop. The disk drive was then cleared and the DAS was restarted and its status checked. The data were later transferred from the laptop to a workstation in the office.

When DASes were found to be non-functional, we attempted to fix the problem in the field. We generally carried a spare DAS in the maintenance vehicle to immediately replace malfunctioning DASes in the field, but during the latter part of the experiment we no longer had spare instruments available. If we could not resolve the problem in the field, we brought the DAS back to the office for inspection, reprogramming or minor repairs. If fixed, we put the DAS back at the site or used it as a spare to replace the next broken DAS. The number of functioning instruments decreased throughout the duration of the experiment, and some sites have time gaps in which a DAS was not present. Appendix A contains a chart that lists the stations, the days each station was functioning, the days the data were downloaded, and the size of the data file that was downloaded in the field.

We removed 50 instruments in late April and early May to move to the San Juan Islands, Washington State, and southwest Canada for another experiment (Brocher et al., 2003). These instruments were taken from throughout the array so that we could maintain the same aerial coverage with fewer instruments. All remaining instruments were removed by May $27^{\text {th }}$ to ship back to IRIS on June $1^{\text {st }}$.

\section{DATA RECOVERY}

The chart in Appendix A summarizes our instrument history and data recovery at each site. Figure 4 shows a summary of the number of stations that recorded each event.

We recovered about $83 \%$ of the data that potentially could have been collected by the instruments. The data recovery percentage is relatively low because it was often two or three weeks between instrument failure and our next visit. The most common problem was power loss because of homeowners accidentally turning off the power to the outdoor outlets, or because of broken power ports on the DASes. In the latter case the instrument would appear in the field to be functioning properly but the bad power port prevented the battery from charging, resulting in battery drain 4 to 5 days later.

Of the data recovered, about $9 \%$ had little or no GPS information from which to do accurate timing corrections, and about 2\% had obvious sensor problems (Appendix A). The GPS clocks gave us numerous problems because of malfunctions or few locks due to limited sky coverage. During our initial site inspection we used a small GPS unit

to check for satellite visibility, but this hand-held unit apparently had far better satellite 
tracking capabilities than the GPS units within the DASes. Sensor problems were primarily due to bad channels (broken wires, flooding), although one site (site \#71) was set on a concrete floor for several weeks before being buried outside of the building (in drier conditions).

About 70 to 75 instruments recorded each event (Figure 4), until we began removing instruments in mid April. The percentage of sites with working instruments gradually declined through the experiment as equipment irrecoverably failed, and the number of instruments decreases rapidly beginning in mid April when we began removing them to send to the other experiment.

\section{GEOPHONE CALIBRATION}

During the experiment, every geophone was calibrated in situ to determine its instrument response (fundamental frequency, $\%$ of critical damping, sensitivity). The calibration process is described in Rogers et al. (1995). The seismometer mass was pulled to the side of the instrument by sending a current into the coil; the mass was held there for several seconds and then released. This was repeated for each direction of each axis (6 measurements total). The resulting response curves were recorded and modeled to obtain an estimate of the resonant frequency, damping and sensitivity for each component. These results are listed in Table 2. In some cases, the calibration pulse was larger than the dynamic range of the recorder, resulting in a clipped calibration pulse (listed in Table 2). This clipping was not recognized in the field because the calibration software mislabeled the calibration pulse and the best-fit model pulse, leading us to the mistaken assumption that the calibration pulse was good but the software had problems with the model. The geophones had been removed before we discovered this problem, so the clipped calibration values have an unknown error. Sixty of the 87 geophones had successful calibrations of the horizontal channels, which were the channels we were interested in for this site response study. The USGS geophones, because they had resistors that were incompatible with the calibration software, were calibrated in the USGS seismology lab in Menlo Park, CA, about 3 weeks after the experiment ended. These values are included for the USGS geophones in Table 2.

\section{DATA PROCESSING}

Data were transferred from the field laptops to a Sun Microsystems workstation, and traces were extracted using the standard PASSCAL software routine "ref2segy". This produced 1-hour SEGY traces plus a log file. Data were quality checked by looking at the log file and plotting the traces on the workstation screen.

Timing corrections were applied using the "refrate" and "clockcor" programs. The refrate program produced the PASSCAL Correction Format (PCF) file, which was inspected with the "clockview" program to see whether there were timing errors. The timing quality varied from the GPS regularly locking every hour for the duration of the experiment to having few to no locks during each 2-week period. Timing corrections were calculated from the log files using the "refrate" program, but the data suffered from numerous "1-s bugs" in which the clock jumps $1 \mathrm{sec}$ and then resets itself at some later time. Timing corrections to remove most of these 1 -s bugs were automatically calculated 
in the "refrate" program, but there were numerous instances where the "refrate" program did not properly handle the errors. If these timing errors occurred during an event that we were saving (Tables 3-5), we hand-edited the timing correction file (PCF file) to attempt to fix the timing errors. When 1-s bugs were improperly handled by the refrate program (i.e. timing errors) but did not coincide with an earthquake, we left the errors in the timing correction files (PCF files) because we did not have time to individually correct all of these errors. Thus, a small percentage (we estimate about 2\%) of our data that does not coincide with one of our events may have timing errors of up to $1 \mathrm{sec}$ that we did not attempt to fix.

After fixing the timing corrections up to a certain date, we concatenated all of the PCF files for each instrument into a composite PCF file named by the date (i.e., MAR_8.PCF). This composite file was copied into the daily data directories, and we used the "clockcor" program to apply the timing corrections. The output from the "clockcor" program was directed to the file 'clockcor.out', which lists the timing correction applied to each data trace.

All local events and local quarry blasts above coda magnitude 1.5, as well as some smaller events that were prominent on the Pacific Northwest Seismic Network, (PNSN) were saved as 5-minute $(300 \mathrm{sec})$ traces. A total of 68 bcal earthquakes were archived (Fig. 5, Table 3), as well as 48 local quarry blasts (Table 4). The traces were started 60 to $90 \mathrm{sec}$ before the origin time of the event.

For teleseisms, records from all events larger than magnitude 5.5 that occurred anywhere on Earth were cut and saved. A total of 143 teleseisms were archived (Fig. 6, Table 5). In addition, smaller teleseisms that were nearby or had prominent arrivals on the PNSN stations were saved.

For all teleseisms less than M7.0, a 1-hour record was saved beginning at the origin time of the event. For earthquakes with magnitudes greater than 7.0, two hours of data beginning at the origin time were cut and saved in two, one-hour records.

\section{DATA QUALITY}

Local events of magnitude 1.9 and above were well recorded if they occurred within the array or on its perimeter (Figs. 7-14), and magnitude 2.3 and larger events within about $50 \mathrm{~km}$ of the array were well recorded (e.g., Figs. 8a, 8b, 9a, 13a). Figures 7-14 show the local events with a $0.5-1-8-16 \mathrm{~Hz}$ trapezoidal bandpass filter. The quarry blasts, most of which were from the Centralia mine $\sim 100 \mathrm{~km}$ southwest of the array, were rarely visible on the array.

For teleseisms, M6.6 and greater events often had a good P-wave signal-to-noise ratio from periods of about $10 \mathrm{sec}$ to $1 \mathrm{sec}$. The S-wave arrivals were near or below noise levels for teleseismic events below M7.0. The largest teleseisms are shown in figures 15 to 20 with a $0.05-0.1-0.8-1.6 \mathrm{~Hz}$ trapezoidal bandpass filter. Although events less than M6.5 were not obvious on the records, we cut and archived events down to M5.5 under the assumption that stacking could be used to view these data. 


\section{DATA FORMAT AND ARCHIVE}

Data are archived in two forms: as 1-hour traces for each instrument for the duration of the experiment, and as cut records of the individual earthquakes and blasts. The former are standard PASSCAL SEGY seismic traces tarred in a directory format, with each day being a separate directory. Within each day's directory are 72 traces for each instrument ( 24 hours times 3 components), for a total of about 5400 traces per day ( 75 instruments times 72 traces). The archived data have had timing corrections applied, and the timing correction file (PCF) is included with each daily directory. Each archive tape contains 3 days of data (about 18 gbytes) except the last two, which contain a greater number of days because there were fewer instruments near the end of the experiment. Also on every archive tape is a main directory with all of the LOG files and all of the PCF files for the individual instruments.

The event records are archived as event directories containing 5-min (local) or 1 hour (teleseism) traces, three per instrument (3 components). These data are in PASSCAL SEGY format with timing corrections applied.

The trace header values are described in Table 6, and include several nonstandard entries. Specifically, the receiver latitude and longitude header entries are in the form of decimal degrees multiplied by 3600 to make them integers (divide by 3600 to return latitude and longitude values. We also put the UTM Easting and Northing of the receiver into the "datumElevRec" and "datumElevSource" header locations as 4-byte integers. There are no source locations in the headers.

Traces from the larger events (Figs. 5-18) also were combined into commonsource gathers stored as industry-standard SEGY data suitable for reading with seismic reflection or refraction data processing software. The trace lengths in these gathers are limited to 32,767 samples by the 16-bit SEGY header, so we resampled the teleseism data to 10 samples/sec and made the gathers a slightly shortened version of the teleseisms (3276 sec; $54.6 \mathrm{~min}$ ). Because the sample rate in the trace headers is limited to a 2-byte integer (32,767 microsec; 3276 millisec), the 100,000 microsecond sample rate was set to 10,000 microsec (10 millisec rather than the true 100 millisec sample rate). Thus, the time scales on these SEGY gathers are a factor of 10 smaller than the true time scale.

\section{DATA AVAILABILITY}

Tape copies of the SEGY data may be ordered via the World Wide Web from the IRIS Data Management System (DMS) in Seattle, Washington. The current web site address of the IRIS Consortium is www.iris.edu. The current email address for the IRIS DMS is webmaster@iris.washington.edu.

In addition to the archival data tapes, the data set contains a CD ROM with Microsoft Excel databases describing:

1) station locations with a calendar showing visits and data recovered [Appendix $\mathrm{A}]$

2) a detailed, 1-page description of each site (contact information, description of seismometer location, instrument numbers, records of site visits), and

3) the data quality control (QC) spreadsheet used during the experiment, which lists the start and end times for each station download, and a list of any problems found during the QC process [data_archive.xls]. 
Also on the $\mathrm{CD}$ are postscript images of the calibration test results ( $\sim 522$ pages).

\section{ACKNOWLEDGMENTS}

Mary Templeton, Steve Azevedo, Marcos Alvarez and Noel Barstow of IRIS PASSCAL provided field support, technical assistance, and logistical support. In addition to the USGS staff, the following volunteers helped deploy the seismometers: Eric Baer, Peter Burkholder, Shane Detweiler, Richard Galster, Greg Geehan, David Hay, Tom Hay, Mike Hickey, Daniel Huang, Lara Linde, Dave McCormack, Mark Molinari, Roger Nye, Charles Oppelt, Ana Pierson, Anne Udaloy, David B. Williams.

The deployment staging area was provided by North Seattle Community College, as was the storage space for equipment cases during the 4 months of the experiment. We thank Dr. Ron LaFayette, President of North Seattle Community College (NSCC), and Mr. Allan Ward, Vice President of Administrative Services, for allowing us to use these facilities. Mr. Larry Raaen, Construction Coordinator, and Mr. Wally Fosmore, Manager of Facilities Operations at NSCC prepared the facilities and provided ongoing logistical support during the duration of the experiment.

Bob Frost and Ana Pierson, both volunteers, spent many days carrying out the geophone calibration process. Mike Flanagan and Shannon Casseia, student volunteers from NSCC, devoted many days to maintaining the instruments and helping with the database. Russell Sell, USGS, Menlo Park, provided the USGS geophones and relevant calibration information. We thank the many landowners whose properties were used during the experiment or who volunteered the use of properties that we chose not to use. Amy Wright of the PNSN provided lists of events that were visible on the permanent network. Bill Steele of the Pacific Northwest Seismology Laboratory handled media coverage and provided logistical support. We thank Mary Templeton for reviewing this manuscript.

\section{REFERENCES}

Brocher, T.M., T. Parsons, K.C. Creager, R.S. Crosson, N.P. Symons, G.D. Spence, B.C. Zelt, P.T.C. Hammer, R.D. Hyndman, D.C. Mosher, A.M. Trehu, K.C. Miller, M.A. Fisher, T.L. Pratt, M.G. Alvarez, B.C. Beaudoin, K.E. Louden, and C.S. Weaver, 1999, Wide-angle seismic recordings from the 1998 seismic hazards investigation of Puget Sound (SHIPS), western Washington and British Columbia, U.S. Geological Survey Open-File Report 99-314, 110 p.

Brocher, T.M., T. L. Pratt, K. C. Miller, A. M. Tréhu, C. M. Snelson, C. S. Weaver, K. S. Creager, R. S. Crosson, U. S. ten Brink, M. G. Alvarez, S. H. Harder and I. Asudeh, 2000a, Report for explosion and earthquake data acquired in the 1999 Seismic Hazard Investigation of Puget Sound (SHIPS), Washington, U. S. Geol. Surv. Open-File Report 00-318, 85 p.

Brocher, T. M., T. L. Pratt, K. C. Creager, R. S. Crosson, W. P. Steele, C. S. Weaver, A. D. Frankel, A. M. Tréhu, C. M. Snelson, K. C. Miller, S. H. Harder and U. S. ten Brink, 2000b, Urban seismic experiments investigate Seattle fault and basin, EOS Trans. Am. Geophys. Union, 81, 545-552.

Brocher, T. M., T. Parsons, R. J. Blakely, N. I. Christensen, M. A. Fisher, R. E. Wells, and SHIPS working group 2001a, Upper crustal structure in Puget Lowland, Washington: Results from the 1998 Seismic Hazards Investigation in Puget Sound, J. Geophys. Res. 106, 13541-13564.

Brocher, T. M., T.L. Pratt, and Dry SHIPS Working Group 2001b, Dry SHIPS recordings of the Chi-Chi earthquake, Seattle, Washington, Bull. Seismol. Soc. Am. 96, 1395.

Brocher, T.M., T.L. Pratt, G.D. Spence, M. Reidel, and R.D. Hyndman R.D., 2003, Wide-angle seismic recordings from the 2002 Georgia Basin geohazards initiative, northwestern Washington and British Columbia, U. S. Geological Survey Open-File Report, 03-160, 35 p. 
Fisher, M.A., T.M. Brocher, R.D. Hyndman, A.M. Trehu, C.S. Weaver, K.C. Creager, R.S. Crosson, T. Parsons, A.K. Cooper, D. Mosher, G. Spence, B.C. Zelt, P.T. Hammer, U.S. ten Brink, T.L. Pratt, K.C. Miller, J.R. Childs, G.R. Cochrane, S. Chopra and R. Walia, 1999, Seismic survey probes urban earthquake hazards in Pacific Northwest, EOS Trans. American Geophys. Union, 80, no. 2, 13-17.

Johnson, S.Y., C.J. Potter and J.M. Armentrout, 1994, Origin and evolution of the Seattle fault and Seattle basin, Washington, Geology, 22, 71-74.

PASSCAL, 1991, Users Guide, A guide to planning experiments using PASSCAL instruments: IRIS, 28 p.

Pratt, T. L., S. Johnson, C. Potter, W. Stephenson and C. Finn, 1997, Seismic reflection images beneath Puget Sound, western Washington State: the Puget lowland thrust sheet hypothesis, J. Geophys. Res. 102, 27469-27489.

Pratt, T.L., C.S. Weaver, T.M. Brocher, T. Parsons, M.A. Fisher, K.C. Creager, R.S. Crosson, R.D. Hyndman, G. Spence, A.M. Trehu, K.C. Miller and U.S. ten Brink, 2002, Understanding the seismotectonics of the Cascadia subduction zone: Overview and recent seismic work, in Fujinawa, Y., and Toshida, A., editors, Seismotectonics in Convergent Plate Boundary, Terra Scientific Publishing, Tokyo, p. 25-36.

Pratt, T.L., T.M. Brocher, C.S. Weaver, K.C. Miller, A.M. Trehu, K.C. Creager and R.S. Crosson, 2003, Amplification of seismic waves by the Seattle basin, Washington State, Bulletin of the Seismological Society of America, 93, p. 533-545.

Rogers, P.W., A.J. Martin, M.C. Robertson, M.M. Hsu and D.B. Harris, 1995, Signal-coil calibration of electromagnetic seismometers, Bulletin of the Seismological Society of America, 85, p. 845-850.

Snelson, C.M., 2001, Investigating crustal structure in western Washington and in the Rocky Mountains: Implications for seismic hazards and crustal growth, Ph.D. Dissertation, The University of Texas at El Paso, El Paso, Texas, 234 pp.

VanWagoner, T.M., R.S. Crosson, K.C. Creager, G. Medema, L. Preston, N.P. Symons and T.M. Brocher, 2002, Crustal structure and relocated earthquakes in the Puget Lowland, Washington, from highresolution seismic tomography, Journal of Geophysical Research, 107, no. 12, p. 22-1 - 22-23. 
TABLE 1: SEISMOMETER SITES

\begin{tabular}{|c|c|c|c|c|c|c|c|c|c|}
\hline \begin{tabular}{|c} 
Site \\
no.
\end{tabular} & $\begin{array}{c}\text { Latitude } \\
\text { (degrees } \\
\text { west) }\end{array}$ & $\begin{array}{c}\text { Longitude } \\
\text { (degrees } \\
\text { north) }\end{array}$ & $\begin{array}{c}\text { UTM } \\
\text { Easting } \\
(\mathrm{m})\end{array}$ & $\begin{array}{c}\text { UTM } \\
\text { Northing } \\
(\mathrm{m}) \\
\end{array}$ & $\begin{array}{r}\text { Elev } \\
(\mathrm{m})\end{array}$ & number & L-22 no. & CITY & Zip \\
\hline 1 & -122.3157 & 47.9178 & 551132 & 5307174 & 25 & 7616 & 1489-L/765-L & Mukilteo & 98275 \\
\hline 2 & -122.3247 & 47.8444 & 550243 & 5299028 & 95 & 7348 & 484-L & Edmonds & 98026 \\
\hline 3 & -122.3502 & 47.7925 & 548669 & 5293228 & 138 & 7285 & 963-L & Edmonds & 98026 \\
\hline 4 & -122.3690 & 47.7809 & 547270 & 5291921 & 124 & 7284 & 1514-L & Edmonds & 98020 \\
\hline 5 & -122.3325 & 47.7322 & 550053 & 5286532 & 138 & 7432 & 736-L & Seattle & 98133 \\
\hline 6 & -122.3737 & 47.7069 & 546982 & 5283696 & 92 & 7335 & 977-L & Seattle & 98177 \\
\hline 7 & -122.3587 & 47.7303 & 548088 & 5286303 & 149 & 7626 & 459-L & Seattle & 98177 \\
\hline 9 & -122.3347 & 47.6930 & 549926 & 5282176 & 84 & 7629 & 454-L & Seattle & 98103 \\
\hline 10 & -122.2925 & 47.6878 & 553099 & 5281630 & 101 & 7281 & 512-L & Seattle & 98115 \\
\hline 11 & -122.2793 & 47.7008 & 554074 & 5283078 & 18 & 7343 & 748-L & Seattle & 98115 \\
\hline 12 & -122.3953 & 47.6749 & 545394 & 5280124 & 47 & 7457 & 533-L & Seattle & 98107 \\
\hline 13 & -122.3560 & 47.6813 & 548335 & 5280856 & 103 & 7433 & 527-L & Seattle & 98103 \\
\hline 14 & -122.2736 & 47.6828 & 554523 & 5281077 & 105 & 7443 & 971-L & Seattle & 98115 \\
\hline 15 & -122.3156 & 47.6691 & 551377 & 5279532 & 61 & 7354 & 1503-L & Seattle & 98105 \\
\hline 16 & -122.2562 & 47.6713 & 555841 & 5279815 & 29 & 7439 & 471-L & Seattle & 98105 \\
\hline 18 & -122.3684 & 47.6447 & 547436 & 5276786 & 94 & 6088 & 542-L & Seattle & 98119 \\
\hline 19 & -122.3181 & 47.6416 & 551219 & 5276471 & 61 & 6096 & 522-L & Seattle & 98102 \\
\hline 20 & -122.3455 & 47.6313 & 549174 & 5275316 & 58 & 6042 & 541-L & Seattle & 98109 \\
\hline 21 & -122.2830 & 47.6351 & 553866 & 5275777 & 19 & 7462 & 994-L & Seattle & 98112 \\
\hline 22 & -122.3173 & 47.6228 & 551298 & 5274382 & 111 & 7365 & 728-L & Seattle & 98102 \\
\hline 23 & -122.2868 & 47.6102 & 553606 & 5273005 & 79 & 7429 & 524-L & Seattle & 98122 \\
\hline 25 & -122.2913 & 47.5815 & 553293 & 5269809 & 53 & 7594 & 526-L & Seattle & 98144 \\
\hline 27 & -122.3164 & 47.5696 & 551418 & 5268469 & 90 & $7090 / 7451$ & 956-L & Seattle & 98108 \\
\hline 28 & -122.2728 & 47.5657 & 554698 & 5268064 & 26 & $6021 / 6084$ & 531-L & Seattle & 98118 \\
\hline 29 & -122.3755 & 47.5531 & 546985 & 5266605 & 115 & 7048 & 746-L & Seattle & 98126 \\
\hline 30 & -122.2989 & 47.5567 & 552750 & 5267050 & 104 & 7317 & 538-L & Seattle & 98108 \\
\hline 31 & -122.2628 & 47.5544 & 555469 & 5266823 & 23 & 7449 & 535-L & Seattle & 98118 \\
\hline
\end{tabular}




\begin{tabular}{|c|c|c|c|c|c|c|c|c|c|}
\hline 32 & -122.3855 & 47.5331 & 546255 & 5264377 & 128 & 7079 & $724-\mathrm{L}$ & Seattle & 98136 \\
\hline 33 & -122.3452 & 47.5282 & 549294 & 5263851 & 129 & 7081 & $448-\mathrm{L}$ & Seattle & 98106 \\
\hline 34 & -122.3835 & 47.5119 & 546420 & 5262016 & 125 & 7065 & 967-L & Seattle & 98146 \\
\hline 35 & -122.3580 & 47.4710 & 548379 & 5257488 & 113 & 7279 & 953-L & Burien & 98166 \\
\hline 36 & -122.3150 & 47.4150 & 551674 & 5251292 & 79 & 7450 & $458-\mathrm{L}$ & Des Moines & 98198 \\
\hline 37 & -122.3710 & 47.2960 & 547557 & 5238032 & 130 & 7445 & 462-L & Federal Way & 98093 \\
\hline 38 & -122.2850 & 47.3390 & 554015 & 5242867 & 142 & $7467 / 6111 / 7608$ & 507-L & Auburn & 98001 \\
\hline 39 & -122.7356 & 47.6852 & 519840 & 5281131 & 65 & 7331 & $742-\mathrm{L}$ & Silverdale & 98383 \\
\hline 40 & -122.5770 & 47.4910 & 531864 & 5259598 & 87 & 7596/7303 & 491-L & Port Orchard & 98367 \\
\hline 41 & -122.6142 & 47.6610 & 528971 & 5278476 & 18 & 7597 & 747-L & Poulsbo & 98370 \\
\hline 42 & -122.5447 & 47.6654 & 534183 & 5278993 & 75 & 7333 & 643-L & Bainbridge Island & 98110 \\
\hline 43 & -122.1942 & 47.6539 & 560510 & 5277933 & 97 & 7316 & 463-L & Kirkland & 98033 \\
\hline 44 & -122.1614 & 47.6633 & 562961 & 5278997 & 142 & 7437 & 1486-L & Kirkland & 98033 \\
\hline 45 & -122.1106 & 47.6430 & 566806 & 5276790 & 48 & 7601 & $640-\mathrm{L}$ & Redmond & 98052 \\
\hline 46 & -121.9117 & 47.6449 & 581740 & 5277185 & 26 & 7620 & 949-L & Carnation & 98014 \\
\hline 50 & -122.3000 & 47.8877 & 552333 & 5303839 & 155 & 7460 & 1508-L & Mukilteo & 98275 \\
\hline 51 & -122.3126 & 47.4889 & 551785 & 5259502 & 116 & 7458 & 537-L & Seattle & 98168 \\
\hline 52 & -122.3490 & 47.4560 & 549072 & 5255827 & 118 & 7466 & 957-L & Burien & 98166 \\
\hline 53 & -122.2940 & 47.3000 & 553375 & 5238526 & 122 & 7610 & 968-L & Auburn & 98001 \\
\hline 54 & -122.3083 & 47.6047 & 551993 & 5272382 & 99 & 7098 & 523-L & Seattle & 98122 \\
\hline 56 & -122.2589 & 47.5125 & 555802 & 5262163 & 76 & $7326 / 6126$ & 536-L & Seattle & 98118 \\
\hline 57 & -122.3208 & 47.5280 & 551127 & 5263841 & 5 & 7103 & 951-L & Seattle & 98108 \\
\hline 58 & -122.3267 & 47.5539 & 550659 & 5266717 & 5 & 7091 & 493-L & Seattle & 98134 \\
\hline 59 & -122.3711 & 47.6703 & 547214 & 5279634 & 26 & 6019 & $528-\mathrm{L}$ & Seattle & 98107 \\
\hline 60 & -122.2854 & 47.7208 & 553592 & 5285299 & 79 & 7280 & 733-L & Seattle & 98125 \\
\hline 61 & -122.2839 & 47.6601 & 553772 & 5278555 & 17 & 7344 & $950-\mathrm{L}$ & Seattle & 98105 \\
\hline 62 & -122.3090 & 47.3720 & 552170 & 5246518 & 84 & 7448 & 743-L & Des Moines & 98198 \\
\hline 63 & -122.4051 & 47.6375 & 544686 & 5275965 & 69 & 6039/6085 & 539-L & Seattle & 98199 \\
\hline 64 & -122.9731 & 47.7195 & 502020 & 5284909 & 51 & 7319 & $465-\mathrm{L}$ & Brinnon & 98320 \\
\hline 65 & -122.9310 & 47.7078 & 505173 & 5283602 & 37 & 7294 & $722-\mathrm{L}$ & Brinnon & 98320 \\
\hline 66 & -122.9035 & 47.6883 & 507246 & 5281444 & 10 & 7618 & 861-L & Brinnon & 98320 \\
\hline 67 & -122.7984 & 47.7092 & 515121 & 5283779 & 128 & 7591 & $727-\mathrm{L}$ & Quilcene & 98376 \\
\hline
\end{tabular}




\begin{tabular}{|c|c|c|c|c|c|c|c|c|c|}
\hline 68 & -122.7945 & 47.6948 & 515419 & 5282177 & 86 & 7595 & 975-L & Quilcene & 98376 \\
\hline 71 & -122.5785 & 47.6656 & 531648 & 5278997 & 40 & 7441 & 1506-L & Bainbridge Island & 98110 \\
\hline 74 & -122.0930 & 47.6644 & 568097 & 5279184 & 53 & 7602 & 1490-L & Redmond & 98052 \\
\hline 75 & -122.0418 & 47.6626 & 571946 & 5279022 & 111 & 7328 & 498-L & Redmond & 98053 \\
\hline 76 & -121.9927 & 47.6760 & 575610 & 5280564 & 179 & 7302 & $495-\mathrm{L}$ & Redmond & 98053 \\
\hline 77 & -121.9532 & 47.6547 & 578608 & 5278235 & 111 & $7617 / 6119$ & 497-L & Carnation & 98014 \\
\hline 79 & -121.8295 & 47.6589 & 587894 & 5278832 & 226 & 7605 & $451-\mathrm{L}$ & Carnation & 98014 \\
\hline 80 & -121.7614 & 47.6535 & 593014 & 5278317 & 335 & 7295 & 1499-L & Carnation & 98014 \\
\hline 81 & -121.7119 & 47.6536 & 596733 & 5278386 & 417 & $7608 / 7327$ & 958-L & Carnation & 98014 \\
\hline 82 & -122.1428 & 47.7462 & 564256 & 5288228 & 67 & 7619 & 473-L & Woodinville & 98072 \\
\hline 83 & -122.1683 & 47.7899 & 562291 & 5293066 & 118 & 7444 & $496-\mathrm{L}$ & Bothell & 98021 \\
\hline 84 & -122.1459 & 47.5935 & 564216 & 5271258 & 111 & 7453 & $718-\mathrm{L}$ & Bellevue & 98007 \\
\hline 85 & -122.1447 & 47.5573 & 564351 & 5267229 & 298 & 7352 & 464-L & Bellevue & 98006 \\
\hline 96 & -122.3989 & 47.6902 & 545107 & 5281827 & 86 & $7336 / 7596$ & $450-\mathrm{L}$ & Seattle & 98117 \\
\hline 98 & -122.3396 & 47.6635 & 549582 & 5278894 & 66 & 6132 & $529-\mathrm{L}$ & Seattle & 98103 \\
\hline 111 & -122.4033 & 47.5780 & 544877 & 5269348 & 30 & 7107 & 959-L & Seattle & 98116 \\
\hline 113 & -122.3900 & 47.5644 & 545889 & 5267852 & 94 & 7064 & $962-L$ & Seattle & 98116 \\
\hline 118 & -122.6592 & 47.6780 & 525578 & 5280347 & 68 & 7446 & 504-L & Poulsbo & 98370 \\
\hline 143 & -122.5630 & 47.7060 & 532783 & 5283502 & 33 & 7325 & 1502-L & Bainbridge Island & 98110 \\
\hline 144 & -122.5079 & 47.6886 & 536929 & 5281589 & 20 & 7630 & 966-L & Bainbridge Island & 98110 \\
\hline 148 & -122.1615 & 47.7213 & 562889 & 5285451 & 106 & 7442 & 485-L & Kirkland & 98034 \\
\hline 169 & -122.1386 & 47.6681 & 564667 & 5279555 & 82 & 7283 & 461-L & Redmond & 98052 \\
\hline 181 & -122.5682 & 47.8094 & 532332 & 5294982 & 59 & 7609 & 1496-L & Kingston & 98346 \\
\hline 182 & -122.5844 & 47.8555 & 531091 & 5300106 & 15 & 7299/7296 & 457-L & Poulsbo & 98370 \\
\hline 183 & -122.1503 & 47.5311 & 563961 & 5264318 & 221 & 7288 & 479-L & Newcastle & 98059 \\
\hline 186 & -122.5479 & 47.6370 & 533960 & 5275833 & 63 & 7599 & 483-L & Bainbridge Island & 98110 \\
\hline 188 & -122.5480 & 47.5750 & 533994 & 5268944 & 7 & 7431 & 644-L & Port Orchard & 98366 \\
\hline 189 & -122.3709 & 47.8162 & 547098 & 5295848 & 25 & 7355 & $1487-\mathrm{L}$ & Edmonds & 98020 \\
\hline
\end{tabular}


TABLE 2: GEOPHONE CALIBRATION RESULTS

(bold = bad calibrations due to clipped pulses)

\begin{tabular}{|c|c|c|c|c|c|c|c|c|c|c|c|c|c|c|c|c|}
\hline \multirow{2}{*}{\begin{tabular}{|l|} 
calib. \\
date \\
ymmdd
\end{tabular}} & statn geoph & \multirow{2}{*}{$\begin{array}{l}\text { sensor } \\
\text { type }\end{array}$} & \multirow{2}{*}{$\begin{array}{l}\text { sensor } \\
\text { no. }\end{array}$} & \multirow{2}{*}{$\begin{array}{l}\text { comp. } \\
\mathrm{L}=\mathrm{N}-\mathrm{S} \\
\mathrm{T}=\mathrm{E}-\mathrm{W}\end{array}$} & \multirow{2}{*}{$\begin{array}{l}\text { polarity } \\
P=\text { pos } \\
N=\text { neg }\end{array}$} & \multirow[b]{2}{*}{ Freq } & \multirow[b]{2}{*}{$\begin{array}{l}\text { period } \\
\text { (sec) }\end{array}$} & \multirow[b]{2}{*}{$\begin{array}{l}\text { damp } \\
\text { (\%crit) }\end{array}$} & \multirow{2}{*}{\multicolumn{2}{|c|}{$\begin{array}{ll}\text { Snstvty } & 0=\text { no } \\
\mathrm{V} / \mathrm{m} / \mathrm{s} & 1=\text { some } \\
& 2=\text { lots }\end{array}$}} & \multicolumn{3}{|c|}{ average of $\mathrm{P}+\mathrm{N}$} & \multicolumn{3}{|c|}{ horizontal average } \\
\hline & $\begin{array}{l}\text { owner } \\
\text { Passcal } \\
\text { USGS }\end{array}$ & & & & & & & & & & Freq & $\begin{array}{l}\text { damp } \\
\text { (\%crit) }\end{array}$ & $\begin{array}{l}\text { Snstvty } \\
\mathrm{V} / \mathrm{m} / \mathrm{s}\end{array}$ & $\begin{array}{l}\text { period } \\
\text { Freq }\end{array}$ & $\begin{array}{l}\text { damp } \\
\text { (\%crit) }\end{array}$ & $\begin{array}{l}\text { Snstvty } \\
\mathrm{V} / \mathrm{m} / \mathrm{s}\end{array}$ \\
\hline 20314 & 1PSCL & L22 & L765 & V & $\mathrm{P}$ & 1.969 & 0.508 & 0.761 & 92.9 & & 1.966 & 0.762 & 92.7 & 1.966 & 0.762 & 92.t \\
\hline 20314 & 1PSCL & L22 & L765 & V & $\mathrm{N}$ & 1.963 & 0.509 & 0.763 & 92.6 & & 2 & & & & & \\
\hline 20314 & $1 \mathrm{PSCL}$ & L22 & L765 & $\mathrm{L}$ & $\mathrm{P}$ & 2.186 & 0.457 & 0.695 & 89.1 & & 2.172 & 0.691 & 89.3 & 2.003 & 0.762 & 91.0 \\
\hline 20314 & $1 \mathrm{PSCL}$ & L22 & L765 & $\mathrm{L}$ & $\mathrm{N}$ & 2.157 & 0.464 & 0.687 & 89.5 & & 0 & & & & & \\
\hline 20314 & 1PSCL & L22 & L765 & $\mathrm{T}$ & $\mathrm{P}$ & 1.817 & 0.550 & 0.837 & 92.6 & & 1.835 & 0.832 & 92.7 & & & \\
\hline 20314 & $1 \mathrm{PSCL}$ & L22 & L765 & $\mathrm{T}$ & $\mathrm{N}$ & 1.853 & 0.540 & 0.827 & 92.7 & & 2 & & & & & \\
\hline 20418 & 2PSCL & L22 & L484 & V & $P$ & 2.104 & 0.475 & 0.773 & 93.6 & & 2.073 & 0.751 & 92.0 & 2.073 & 0.751 & 92.0 \\
\hline 20418 & 2PSCL & L22 & L484 & V & $\mathrm{N}$ & 2.041 & 0.490 & 0.729 & 90.4 & & 0 & & & & & \\
\hline 20418 & 2PSCL & L22 & L484 & $\mathrm{L}$ & $P$ & 2.184 & 0.458 & 0.685 & 87.9 & & 2.100 & 0.670 & 86.5 & 2.101 & 0.673 & 86.0 \\
\hline 20418 & 2PSCL & L22 & L484 & $\mathrm{L}$ & $\mathrm{N}$ & 2.016 & 0.496 & 0.655 & 85.0 & & 0 & & & & & \\
\hline 20418 & 2PSCL & L22 & L484 & $\mathrm{T}$ & $\mathrm{P}$ & 2.178 & 0.459 & 0.693 & 87.3 & & 2.102 & 0.676 & 85.6 & & & \\
\hline 20418 & 2PSCL & L22 & L484 & $\mathrm{T}$ & $\mathrm{N}$ & 2.027 & 0.493 & 0.660 & 83.8 & & 0 & & & & & \\
\hline 20314 & 3PSCL & L22 & L963 & V & $\mathrm{P}$ & 2.016 & 0.496 & 0.756 & 92.3 & & 2.077 & 0.788 & 95.3 & 2.077 & 0.788 & 95.3 \\
\hline 20314 & 3PSCL & L22 & L963 & V & $\mathrm{N}$ & 2.138 & 0.468 & 0.820 & 98.4 & & 1 & & & & & \\
\hline 20314 & $3 \mathrm{PSCL}$ & L22 & L963 & $\mathrm{L}$ & $\mathrm{P}$ & 1.845 & 0.542 & 0.825 & 90.7 & 0 & 1.847 & 0.824 & 90.4 & 2.012 & 0.746 & 88.8 \\
\hline 20314 & 3PSCL & L22 & L963 & $\mathrm{L}$ & $\mathrm{N}$ & 1.849 & 0.541 & 0.823 & 90.1 & & 0 & & & & & \\
\hline 20314 & 3PSCL & L22 & L963 & $\mathrm{T}$ & $\mathrm{P}$ & 2.245 & 0.445 & 0.689 & 88.5 & & 2.176 & 0.668 & 87.1 & & & \\
\hline 20314 & $3 \mathrm{PSCL}$ & L22 & L963 & $\mathrm{T}$ & $\mathrm{N}$ & 2.107 & 0.475 & 0.647 & 85.7 & & 0 & & & & & \\
\hline 20314 & 4 PSCL & L22 & L1514 & V & $\mathrm{P}$ & 1.813 & 0.552 & 0.758 & 89.2 & & 1.833 & 0.763 & 89.9 & 1.833 & 0.763 & 89.9 \\
\hline 20314 & 4PSCL & L22 & L1514 & V & $\mathrm{N}$ & 1.853 & 0.540 & 0.768 & 90.6 & & 2 & & & & & \\
\hline 20314 & 4 PSCL & L22 & L1514 & $\mathrm{L}$ & $P$ & 2.071 & 0.483 & 0.668 & 91.4 & & 2.170 & 0.682 & 93.6 & 2.058 & 0.728 & 93.4 \\
\hline 20314 & $4 \mathrm{PSCL}$ & L22 & L1514 & $\mathrm{L}$ & $\mathrm{N}$ & 2.270 & 0.441 & 0.696 & 95.8 & & 0 & & & & & \\
\hline 20314 & 4 PSCL & L22 & L1514 & $\mathrm{T}$ & $\mathrm{P}$ & 1.935 & 0.517 & 0.766 & 92.7 & & 1.946 & 0.774 & 93.3 & & & \\
\hline 20314 & 4 PSCL & L22 & L1514 & $\mathrm{T}$ & $\mathrm{N}$ & 1.957 & 0.511 & 0.782 & 93.9 & & 2 & & & & & \\
\hline
\end{tabular}




\begin{tabular}{|c|c|c|c|c|c|c|c|c|c|c|c|c|c|c|c|c|}
\hline 20314 & 5PSCL & L22 & L736 & V & $P$ & 1.982 & 0.505 & 0.707 & 89.8 & 0 & 1.963 & 0.703 & 89.4 & 1.963 & 0.703 & 89.4 \\
\hline 20314 & 5PSCL & L22 & L736 & V & $\mathrm{N}$ & 1.944 & 0.514 & 0.699 & 89.0 & 1 & & & & & & \\
\hline 20314 & 5PSCL & L22 & L736 & $\mathrm{L}$ & $\mathrm{P}$ & 2.145 & 0.466 & 0.859 & 99.0 & 0 & 2.080 & 0.834 & 96.9 & 1.899 & 0.859 & 93.4 \\
\hline 20314 & $5 \mathrm{PSCL}$ & L22 & L736 & $\mathrm{L}$ & $\mathrm{N}$ & 2.016 & 0.496 & 0.808 & 94.8 & 1 & & & & & & \\
\hline 20314 & $5 \mathrm{PSCL}$ & L22 & L736 & $\mathrm{T}$ & $\mathrm{P}$ & 1.742 & 0.574 & 0.880 & 90.0 & 1 & 1.718 & 0.885 & 89.9 & & & \\
\hline 20314 & 5PSCL & L22 & L736 & $\mathrm{T}$ & $\mathrm{N}$ & 1.693 & 0.591 & 0.890 & 89.8 & 1 & & & & & & \\
\hline 20222 & 6PSCL & L22 & L977 & V & $P$ & 2.139 & 0.468 & 0.767 & 93.6 & 0 & 2.112 & 0.765 & 92.5 & 2.112 & 0.765 & 92.5 \\
\hline 20222 & 6PSCL & L22 & L977 & V & $\mathrm{N}$ & 2.085 & 0.480 & 0.762 & 91.4 & 0 & & & & & & \\
\hline 20222 & 6PSCL & L22 & L977 & $\mathrm{L}$ & $P$ & 2.136 & 0.468 & 0.776 & 93.2 & 0 & 2.094 & 0.778 & 92.9 & 2.063 & 0.748 & 90.4 \\
\hline 20222 & 6PSCL & L22 & L977 & $\mathrm{L}$ & $\mathrm{N}$ & 2.052 & 0.487 & 0.781 & 92.7 & 0 & & & & & & \\
\hline 20222 & 6PSCL & L22 & L977 & $\mathrm{T}$ & $\mathrm{P}$ & 2.036 & 0.491 & 0.692 & 86.0 & 0 & 2.033 & 0.718 & 88.0 & & & \\
\hline 20222 & $6 \mathrm{PSCL}$ & L22 & L977 & $\mathrm{T}$ & $N$ & 2.030 & 0.493 & 0.743 & 89.9 & 0 & & & & & & \\
\hline 20314 & 7PSCL & L22 & L459 & V & $P$ & 2.102 & 0.476 & 0.814 & 97.1 & 0 & 2.098 & 0.816 & 96.8 & 2.098 & 0.816 & 96.8 \\
\hline 20314 & 7PSCL & L22 & L459 & V & $\mathrm{N}$ & 2.093 & 0.478 & 0.819 & 96.6 & 0 & & & & & & \\
\hline 20314 & 7PSCL & L22 & L459 & $\mathrm{L}$ & $\mathrm{P}$ & 1.847 & 0.541 & 0.802 & 90.1 & 0 & 1.905 & 0.789 & 89.6 & 1.948 & 0.760 & 89.0 \\
\hline 20314 & 7PSCL & L22 & L459 & $\mathrm{L}$ & $\mathrm{N}$ & 1.962 & 0.510 & 0.776 & 89.1 & 0 & & & & & & \\
\hline 20314 & 7PSCL & L22 & L459 & $\mathrm{T}$ & $\mathrm{P}$ & 1.960 & 0.510 & 0.719 & 87.4 & 0 & 1.991 & 0.731 & 88.5 & & & \\
\hline 20314 & $7 \mathrm{PSCL}$ & L22 & L459 & $\mathrm{T}$ & $\mathrm{N}$ & 2.021 & 0.495 & 0.743 & 89.5 & 0 & & & & & & \\
\hline 20222 & 9 PSCL & L22 & L454 & V & $\mathrm{P}$ & 2.206 & 0.453 & 0.603 & 91.0 & 0 & 2.210 & 0.617 & 92.3 & 2.210 & 0.617 & 92.3 \\
\hline 20222 & 9PSCL & L22 & L454 & $\mathrm{V}$ & $\mathrm{N}$ & 2.214 & 0.452 & 0.631 & 93.7 & 2 & & & & & & \\
\hline 20222 & 9PSCL & L22 & L454 & $\mathrm{L}$ & $\mathrm{P}$ & 1.689 & 0.592 & 0.805 & 88.4 & 2 & 1.600 & 0.788 & 85.5 & 1.823 & 0.728 & 88.1 \\
\hline 20222 & 9 PSCL & L22 & L454 & $\mathrm{L}$ & $\mathrm{N}$ & 1.510 & 0.662 & 0.772 & 82.7 & 2 & & & & & & \\
\hline 20222 & $9 \mathrm{PSCL}$ & L22 & L454 & $\mathrm{T}$ & $\mathrm{P}$ & 2.120 & 0.472 & 0.671 & 91.6 & 0 & 2.046 & 0.668 & 90.6 & & & \\
\hline 20222 & 9PSCL & L22 & L454 & $\mathrm{T}$ & $\mathrm{N}$ & 1.973 & 0.507 & 0.665 & 89.7 & 2 & & & & & & \\
\hline 20427 & 10PSCL & L22 & L512 & V & $\mathrm{P}$ & 2.193 & 0.456 & 0.764 & 95.3 & 0 & 2.176 & 0.758 & 94.5 & 2.176 & 0.758 & 94.5 \\
\hline 20427 & 10PSCL & L22 & L512 & V & $\mathrm{N}$ & 2.160 & 0.463 & 0.752 & 93.8 & 0 & & & & & & \\
\hline 20427 & 10PSCL & L22 & L512 & $\mathrm{L}$ & $\mathrm{P}$ & 1.546 & 0.647 & 0.810 & 83.5 & 2 & 1.632 & 0.809 & 84.8 & 1.853 & 0.776 & 88.0 \\
\hline 20427 & 10PSCL & L22 & L512 & $\mathrm{L}$ & $\mathrm{N}$ & 1.718 & 0.582 & 0.808 & 86.1 & 0 & & & & & & \\
\hline 20427 & $10 \mathrm{PSCL}$ & L22 & L512 & $\mathrm{T}$ & $\mathrm{P}$ & 62.500 & 0.016 & 0.166 & 245.0 bad & & 2.075 & 0.743 & 91.3 & & & \\
\hline 20427 & 10PSCL & L22 & L512 & $\mathrm{T}$ & $\mathrm{N}$ & 2.075 & 0.482 & 0.743 & 91.3 & 0 & & & & & & \\
\hline
\end{tabular}




\begin{tabular}{|c|c|c|c|c|c|c|c|c|c|c|c|c|c|c|c|c|}
\hline 20427 & 10PSCL & L22 & L512 & V & $\mathrm{P}$ & 2.211 & 0.452 & 0.764 & 95.3 & 0 & 2.213 & 0.763 & 95.5 & 2.213 & 0.763 & 95.5 \\
\hline 20427 & 10PSCL & L22 & L512 & V & $\mathrm{N}$ & 2.214 & 0.452 & 0.762 & 95.7 & 0 & & & & & & \\
\hline 20427 & 10PSCL & L22 & L512 & $\mathrm{L}$ & $\mathrm{P}$ & 1.576 & 0.635 & 0.810 & 84.4 & 1 & 1.653 & 0.812 & 85.4 & 1.881 & 0.774 & 88.2 \\
\hline 20427 & 10PSCL & L22 & L512 & $\mathrm{L}$ & $\mathrm{N}$ & 1.731 & 0.578 & 0.813 & 86.4 & 0 & & & & & & \\
\hline 20427 & 10PSCL & L22 & L512 & $\mathrm{T}$ & $\mathrm{P}$ & 2.145 & 0.466 & 0.751 & 91.8 & 0 & 2.108 & 0.737 & 91.1 & & & \\
\hline 20427 & 10PSCL & L22 & L512 & $\mathrm{T}$ & $\mathrm{N}$ & 2.072 & 0.483 & 0.723 & 90.3 & 0 & & & & & & \\
\hline 20314 & $11 \mathrm{PSCL}$ & L22 & L748 & V & $P$ & 1.950 & 0.513 & 0.770 & 93.6 & 2 & 1.917 & 0.761 & 92.3 & 1.917 & 0.761 & 92.3 \\
\hline 20314 & $11 \mathrm{PSCL}$ & L22 & L748 & V & $\mathrm{N}$ & 1.884 & 0.531 & 0.751 & 91.1 & 2 & & & & & & \\
\hline 20314 & $11 \mathrm{PSCL}$ & L22 & L748 & $\mathrm{L}$ & $\mathrm{P}$ & 2.117 & 0.472 & 0.706 & 93.6 & 1 & 2.205 & 0.713 & 94.4 & 2.037 & 0.730 & 92.3 \\
\hline 20314 & $11 \mathrm{PSCL}$ & L22 & L748 & $\mathrm{L}$ & $\mathrm{N}$ & 2.293 & 0.436 & 0.720 & 95.2 & 0 & & & & & & \\
\hline 20314 & $11 \mathrm{PSCL}$ & L22 & L748 & $\mathrm{T}$ & $\mathrm{P}$ & 1.885 & 0.531 & 0.755 & 90.9 & 2 & 1.870 & 0.748 & 90.2 & & & \\
\hline 20314 & $11 \mathrm{PSCL}$ & L22 & L748 & $\mathrm{T}$ & $N$ & 1.855 & 0.539 & 0.740 & 89.5 & 2 & & & & & & \\
\hline 20222 & 12 USGS & L22 & L533 & V & $\mathrm{P}$ & 2.100 & 0.476 & 0.819 & 54.4 & 0 & 2.083 & 0.819 & 54.2 & 2.083 & 0.819 & 54.2 \\
\hline 20222 & 12 USGS & L22 & L533 & V & $\mathrm{N}$ & 2.067 & 0.484 & 0.819 & 54.1 & 0 & & & & & & \\
\hline 20222 & 12 USGS & L22 & L533 & $\mathrm{L}$ & $\mathrm{P}$ & 2.133 & 0.469 & 0.792 & 49.3 & 0 & 2.133 & 0.788 & 49.3 & 2.061 & 0.824 & 49.7 \\
\hline 20222 & 12 USGS & L22 & L533 & $\mathrm{L}$ & $\mathrm{N}$ & 2.133 & 0.469 & 0.785 & 49.3 & 0 & & & & & & \\
\hline 20222 & 12 USGS & L22 & L533 & $\mathrm{T}$ & $\mathrm{P}$ & 2.025 & 0.494 & 0.842 & 49.7 & 0 & 1.990 & 0.860 & 50.2 & & & \\
\hline 20222 & 12 USGS & L22 & L533 & $\mathrm{T}$ & $\mathrm{N}$ & 1.955 & 0.512 & 0.879 & 50.7 & 0 & & & & & & \\
\hline 20222 & 13 USGS & L22 & L527 & V & $P$ & 2.232 & 0.448 & 0.891 & 56.2 & 0 & 2.203 & 0.867 & 55.1 & 2.203 & 0.867 & 55.1 \\
\hline 20222 & 13 USGS & L22 & L527 & $\mathrm{V}$ & $\mathrm{N}$ & 2.173 & 0.460 & 0.844 & 53.9 & 0 & & & & & & \\
\hline 20222 & 13 USGS & L22 & L527 & $\mathrm{L}$ & $\mathrm{P}$ & 2.112 & 0.474 & 0.921 & 54.5 & 0 & 2.098 & 0.927 & 54.7 & 2.023 & 0.907 & 54.6 \\
\hline 20222 & 13 USGS & L22 & L527 & $\mathrm{L}$ & $\mathrm{N}$ & 2.084 & 0.480 & 0.933 & 54.8 & 0 & & & & & & \\
\hline 20222 & 13 USGS & L22 & L527 & $\mathrm{T}$ & $\mathrm{P}$ & 1.943 & 0.515 & 0.892 & 54.6 & 0 & 1.948 & 0.886 & 54.4 & & & \\
\hline 20222 & 13USGS & L22 & L527 & $\mathrm{T}$ & $\mathrm{N}$ & 1.952 & 0.512 & 0.880 & 54.3 & 0 & & & & & & \\
\hline 20314 & 14PSCL & L22 & L971 & V & $\mathrm{P}$ & 2.048 & 0.488 & 0.722 & 89.8 & 0 & 2.089 & 0.752 & 92.3 & 2.089 & 0.752 & 92.3 \\
\hline 20314 & 14PSCL & L22 & L971 & V & $\mathrm{N}$ & 2.131 & 0.469 & 0.782 & 94.9 & 0 & & & & & & \\
\hline 20314 & $14 \mathrm{PSCL}$ & L22 & L971 & $\mathrm{L}$ & $\mathrm{P}$ & 1.961 & 0.510 & 0.791 & 89.6 & 0 & 1.913 & 0.777 & 88.7 & 1.830 & 0.797 & 87.7 \\
\hline 20314 & 14PSCL & L22 & L971 & $\mathrm{L}$ & $\mathrm{N}$ & 1.866 & 0.536 & 0.764 & 87.8 & 0 & & & & & & \\
\hline 20314 & $14 \mathrm{PSCL}$ & L22 & L971 & $\mathrm{T}$ & $\mathrm{P}$ & 1.761 & 0.568 & 0.815 & 86.9 & 0 & 1.747 & 0.816 & 86.7 & & & \\
\hline 20314 & 14PSCL & L22 & L971 & $\mathrm{T}$ & $\mathrm{N}$ & 1.734 & 0.577 & 0.818 & 86.4 & 0 & & & & & & \\
\hline
\end{tabular}




\begin{tabular}{|c|c|c|c|c|c|c|c|c|c|c|c|c|c|c|c|c|}
\hline 20222 & 15PSCL & L22 & L1503 & V & $P$ & 1.946 & 0.514 & 0.811 & 94.3 & 2 & 1.940 & 0.793 & 93.4 & 1.940 & 0.793 & 93.4 \\
\hline 20222 & 15PSCL & L22 & L1503 & V & $\mathrm{N}$ & 1.935 & 0.517 & 0.776 & 92.4 & 2 & & & & & & \\
\hline 20222 & 15PSCL & L22 & L1503 & $\mathrm{L}$ & $\mathrm{P}$ & 1.869 & 0.535 & 0.779 & 88.0 & 0 & 1.885 & 0.782 & 88.1 & 2.111 & 0.765 & 93.8 \\
\hline 20222 & 15PSCL & L22 & L1503 & $\mathrm{L}$ & $\mathrm{N}$ & 1.901 & 0.526 & 0.785 & 88.2 & 0 & & & & & & \\
\hline 20222 & $15 \mathrm{PSCL}$ & L22 & L1503 & $\mathrm{T}$ & $\mathrm{P}$ & 2.404 & 0.416 & 0.734 & 99.1 & 0 & 2.338 & 0.748 & 99.6 & & & \\
\hline 20222 & 15PSCL & L22 & L1503 & $\mathrm{T}$ & $\mathrm{N}$ & 2.272 & 0.440 & 0.763 & 100.1 & 1 & & & & & & \\
\hline 20314 & $16 \mathrm{PSCL}$ & L22 & L471 & V & $\mathrm{P}$ & 2.149 & 0.465 & 0.685 & 89.3 & 0 & 2.152 & 0.694 & 90.3 & 2.152 & 0.694 & 90.3 \\
\hline 20314 & $16 \mathrm{PSCL}$ & L22 & L471 & V & $\mathrm{N}$ & 2.155 & 0.464 & 0.702 & 91.4 & 0 & & & & & & \\
\hline 20314 & $16 \mathrm{PSCL}$ & L22 & L471 & $\mathrm{L}$ & $\mathrm{P}$ & 46.948 & 0.021 & 0.016 & 53.9 dead & & 45.404 & 0.071 & 64.0 & 2.439 & 0.563 & 84.0 \\
\hline 20314 & $16 \mathrm{PSCL}$ & L22 & L471 & $\mathrm{L}$ & $\mathrm{N}$ & 43.860 & 0.023 & 0.125 & 74.1 dead & & & & & & & \\
\hline 20314 & $16 \mathrm{PSCL}$ & L22 & L471 & $\mathrm{T}$ & $\mathrm{P}$ & 2.473 & 0.404 & 0.562 & 83.5 & 0 & 2.439 & 0.563 & 84.0 & & & \\
\hline 20314 & $16 \mathrm{PSCL}$ & L22 & L471 & $\mathrm{T}$ & $\mathrm{N}$ & 2.404 & 0.416 & 0.564 & 84.5 & 0 & & & & & & \\
\hline 20314 & $16 \mathrm{PSCL}$ & L22 & L471 & V & $\mathrm{P}$ & 2.208 & 0.453 & 0.696 & 90.9 & 0 & 2.177 & 0.694 & 90.6 & 2.177 & 0.694 & 90.6 \\
\hline 20314 & $16 \mathrm{PSCL}$ & L22 & L471 & V & $\mathrm{N}$ & 2.146 & 0.466 & 0.691 & 90.3 & 0 & & & & & & \\
\hline 20314 & 16PSCL & L22 & L471 & $\mathrm{L}$ & $\mathrm{P}$ & 46.948 & 0.021 & 0.017 & 53.8 dead & & 46.201 & 0.061 & 64.2 & 2.440 & 0.553 & 83.0 \\
\hline 20314 & $16 \mathrm{PSCL}$ & L22 & L471 & $\mathrm{L}$ & $\mathrm{N}$ & 45.455 & 0.022 & 0.104 & 74.5 dead & & & & & & & \\
\hline 20314 & $16 \mathrm{PSCL}$ & L22 & L471 & $\mathrm{T}$ & $\mathrm{P}$ & 2.481 & 0.403 & 0.553 & 82.8 & 0 & 2.440 & 0.553 & 83.0 & & & \\
\hline 20314 & $16 \mathrm{PSCL}$ & L22 & L471 & $\mathrm{T}$ & $\mathrm{N}$ & 2.398 & 0.417 & 0.552 & 83.1 & 0 & & & & & & \\
\hline 20222 & 18 USGS & L22 & L542 & V & $\mathrm{P}$ & 2.058 & 0.486 & 0.762 & 48.3 & 0 & 2.045 & 0.761 & 48.1 & 2.045 & 0.761 & 48.1 \\
\hline 20222 & 18 USGS & L22 & L542 & V & $\mathrm{N}$ & 2.033 & 0.492 & 0.760 & 47.9 & 0 & & & & & & \\
\hline 20222 & 18 USGS & L22 & L542 & $\mathrm{L}$ & $\mathrm{P}$ & 2.027 & 0.493 & 0.809 & 51.4 & 0 & 2.001 & 0.821 & 51.6 & 1.983 & 0.811 & 51.1 \\
\hline 20222 & 18 USGS & L22 & L542 & $\mathrm{L}$ & $\mathrm{N}$ & 1.976 & 0.506 & 0.832 & 51.8 & 0 & & & & & & \\
\hline 20222 & 18 USGS & L22 & L542 & $\mathrm{T}$ & $\mathrm{P}$ & 2.017 & 0.496 & 0.806 & 51.1 & 0 & 1.964 & 0.801 & 50.5 & & & \\
\hline 20222 & 18USGS & L22 & L542 & $\mathrm{T}$ & $\mathrm{N}$ & 1.912 & 0.523 & 0.795 & 49.9 & 0 & & & & & & \\
\hline 20418 & 19 USGS & L22 & L522(552) & V & $\mathrm{P}$ & 1.999 & 0.500 & 0.818 & 51.0 & 0 & 2.004 & 0.818 & 52.4 & 2.004 & 0.818 & 52.4 \\
\hline 20418 & 19 USGS & L22 & L522 & V & $\mathrm{N}$ & 2.010 & 0.498 & 0.818 & 53.8 & 0 & & & & & & \\
\hline 20418 & 19 USGS & L22 & L522 & $\mathrm{L}$ & $\mathrm{P}$ & 1.996 & 0.501 & 0.763 & 51.9 & 0 & 1.993 & 0.768 & 52.5 & 2.112 & 0.758 & 52.1 \\
\hline 20418 & 19 USGS & L22 & L522 & $\mathrm{L}$ & $\mathrm{N}$ & 1.990 & 0.503 & 0.774 & 53.2 & 0 & & & & & & \\
\hline 20418 & 19 USGS & L22 & L522 & $\mathrm{T}$ & $\mathrm{P}$ & 2.247 & 0.445 & 0.751 & 52.0 & 0 & 2.230 & 0.748 & 51.7 & & & \\
\hline 20418 & 19 USGS & L22 & L522 & $\mathrm{T}$ & $\mathrm{N}$ & 2.213 & 0.452 & 0.744 & 51.3 & 0 & & & & & & \\
\hline
\end{tabular}




\begin{tabular}{|c|c|c|c|c|c|c|c|c|c|c|c|c|c|c|c|c|}
\hline 20222 & 20 USGS & L22 & L541 & V & $P$ & 2.261 & 0.442 & 0.844 & 55.6 & 0 & 2.259 & 0.856 & 56.0 & 2.259 & 0.856 & 56.0 \\
\hline 20222 & 20 USGS & L22 & L541 & V & $\mathrm{N}$ & 2.257 & 0.443 & 0.869 & 56.4 & 0 & & & & & & \\
\hline 20222 & 20 USGS & L22 & L541 & $\mathrm{L}$ & $\mathrm{P}$ & 1.982 & 0.505 & 0.815 & 53.5 & 0 & 1.989 & 0.817 & 53.5 & 1.974 & 0.807 & 53.1 \\
\hline 20222 & 20 USGS & L22 & L541 & $\mathrm{L}$ & $\mathrm{N}$ & 1.996 & 0.501 & 0.820 & 53.6 & 0 & & & & & & \\
\hline 20222 & 20 USGS & L22 & L541 & $\mathrm{T}$ & $\mathrm{P}$ & 1.962 & 0.510 & 0.802 & 52.8 & 0 & 1.959 & 0.796 & 52.6 & & & \\
\hline 20222 & 20 USGS & L22 & L541 & $\mathrm{T}$ & $\mathrm{N}$ & 1.956 & 0.511 & 0.790 & 52.3 & 0 & & & & & & \\
\hline 20418 & $21 \mathrm{PSCL}$ & L22 & L974 & V & $P$ & 2.091 & 0.478 & 0.750 & 90.8 & 0 & 2.063 & 0.736 & 89.8 & 2.063 & 0.736 & 89.8 \\
\hline 20418 & 21 PSCL & L22 & L974 & V & $\mathrm{N}$ & 2.035 & 0.492 & 0.722 & 88.7 & 0 & & & & & & \\
\hline 20418 & $21 \mathrm{PSCL}$ & L22 & L974 & $\mathrm{L}$ & $\mathrm{P}$ & 2.521 & 0.397 & 0.569 & 85.0 & 0 & 2.513 & 0.575 & 86.1 & 2.287 & 0.677 & 89.2 \\
\hline 20418 & 21 PSCL & L22 & L974 & $\mathrm{L}$ & $\mathrm{N}$ & 2.506 & 0.399 & 0.582 & 87.2 & 0 & & & & & & \\
\hline 20418 & 21 PSCL & L22 & L974 & $\mathrm{T}$ & $\mathrm{P}$ & 2.107 & 0.475 & 0.779 & 92.4 & 0 & 2.062 & 0.778 & 92.3 & & & \\
\hline 20418 & $21 \mathrm{PSCL}$ & L22 & L974 & $\mathrm{T}$ & $N$ & 2.016 & 0.496 & 0.777 & 92.1 & 0 & & & & & & \\
\hline 20418 & $22 \mathrm{PSCL}$ & L22 & L728 & V & $P$ & 1.957 & 0.511 & 0.717 & 89.4 & 0 & 1.926 & 0.705 & 88.3 & 1.926 & 0.705 & 88.3 \\
\hline 20418 & $22 \mathrm{PSCL}$ & L22 & L728 & V & $\mathrm{N}$ & 1.896 & 0.527 & 0.693 & 87.1 & 0 & & & & & & \\
\hline 20418 & $22 \mathrm{PSCL}$ & L22 & L728 & $\mathrm{L}$ & $\mathrm{P}$ & 2.287 & 0.437 & 0.700 & 89.0 & 0 & 2.216 & 0.694 & 88.2 & 2.231 & 0.639 & 84.8 \\
\hline 20418 & 22 PSCL & L22 & L728 & $\mathrm{L}$ & $\mathrm{N}$ & 2.145 & 0.466 & 0.688 & 87.5 & 0 & & & & & & \\
\hline 20418 & $22 \mathrm{PSCL}$ & L22 & L728 & $\mathrm{T}$ & $\mathrm{P}$ & 2.285 & 0.438 & 0.579 & 80.4 & 0 & 2.245 & 0.583 & 81.4 & & & \\
\hline 20418 & $22 \mathrm{PSCL}$ & L22 & L728 & $\mathrm{T}$ & $\mathrm{N}$ & 2.205 & 0.454 & 0.588 & 82.3 & 0 & & & & & & \\
\hline 20427 & 23 USGS & L22 & L524 & V & $\mathrm{P}$ & 1.925 & 0.520 & 0.802 & 49.0 & 0 & 1.926 & 0.798 & 50.5 & 1.926 & 0.798 & 50.5 \\
\hline 20427 & 23 USGS & L22 & L524 & $\mathrm{V}$ & $\mathrm{N}$ & 1.928 & 0.519 & 0.794 & 52.0 & 0 & & & & & & \\
\hline 20427 & 23 USGS & L22 & L524 & $\mathrm{L}$ & $\mathrm{P}$ & -60.241 & -0.017 & 0.000 & $55.9 \mathrm{bad}$ & & -0.180 & 0.000 & 56.4 & 2.107 & 0.736 & 50.6 \\
\hline 20427 & 23 USGS & L22 & L524 & $\mathrm{L}$ & $\mathrm{N}$ & 59.880 & 0.017 & 0.000 & $56.9 \mathrm{bad}$ & & & & & & & \\
\hline 20427 & 23 USGS & L22 & L524 & $\mathrm{T}$ & $\mathrm{P}$ & 1.981 & 0.505 & 0.781 & 50.8 & 0 & 2.107 & 0.736 & 50.6 & & & \\
\hline 20427 & 23 USGS & L22 & L524 & $\mathrm{T}$ & $\mathrm{N}$ & 2.234 & 0.448 & 0.691 & 50.3 & 0 & & & & & & \\
\hline 20528 & 25 USGS & L22 & L526 & V & $\mathrm{P}$ & 1.963 & 0.510 & 0.786 & 49.5 & 0 & 1.938 & 0.787 & 49.1 & 1.938 & 0.787 & 49.1 \\
\hline 20528 & 25 USGS & L22 & L526 & V & $\mathrm{N}$ & 1.913 & 0.523 & 0.788 & 48.7 & 0 & & & & & & \\
\hline 20528 & 25 USGS & L22 & L526 & $\mathrm{L}$ & $\mathrm{P}$ & 2.050 & 0.488 & 0.758 & 48.4 & 0 & 2.081 & 0.767 & 49.0 & 2.176 & 0.774 & 48.1 \\
\hline 20528 & 25 USGS & L22 & L526 & $\mathrm{L}$ & $\mathrm{N}$ & 2.111 & 0.474 & 0.776 & 49.5 & 0 & & & & & & \\
\hline 20528 & 25 USGS & L22 & L526 & $\mathrm{T}$ & $\mathrm{P}$ & 2.325 & 0.430 & 0.788 & 47.8 & 0 & 2.270 & 0.781 & 47.2 & & & \\
\hline 20528 & 25 USGS & L22 & L526 & $\mathrm{T}$ & $\mathrm{N}$ & 2.216 & 0.451 & 0.775 & 46.7 & 0 & & & & & & \\
\hline
\end{tabular}




\begin{tabular}{|c|c|c|c|c|c|c|c|c|c|c|c|c|c|c|c|c|}
\hline 20314 & 27PSCL & L22 & L956 & V & $P$ & 2.021 & 0.495 & 0.717 & 88.3 & 0 & 2.023 & 0.732 & 88.6 & 2.023 & 0.732 & 88.6 \\
\hline 20314 & 27PSCL & L22 & L956 & V & $\mathrm{N}$ & 2.026 & 0.494 & 0.747 & 89.0 & 0 & & & & & & \\
\hline 20314 & 27PSCL & L22 & L956 & $\mathrm{L}$ & $\mathrm{P}$ & 47.619 & 0.021 & 0.062 & 73.8dead & & 47.848 & 0.042 & 63.4 & 3.096 & 0.446 & 74.6 \\
\hline 20314 & 27PSCL & L22 & L956 & $\mathrm{L}$ & $\mathrm{N}$ & 48.077 & 0.021 & 0.022 & 53.0 dead & & & & & & & \\
\hline 20314 & 27PSCL & L22 & L956 & $\mathrm{T}$ & $\mathrm{P}$ & 3.074 & 0.325 & 0.446 & 66.6 & 0 & 3.096 & 0.446 & 74.6 & & & \\
\hline 20314 & $27 \mathrm{PSCL}$ & L22 & L956 & $\mathrm{T}$ & $\mathrm{N}$ & 3.117 & 0.321 & 0.447 & 82.7 & 0 & & & & & & \\
\hline 20528 & 28 USGS & L22 & L531 & V & $\mathrm{P}$ & 1.997 & 0.501 & 0.739 & 44.9 & 0 & 1.985 & 0.727 & 44.2 & 1.985 & 0.727 & 44.2 \\
\hline 20528 & 28 USGS & L22 & L531 & V & $\mathrm{N}$ & 1.973 & 0.507 & 0.715 & 43.5 & 0 & & & & & & \\
\hline 20528 & 28 USGS & L22 & L531 & $\mathrm{L}$ & $\mathrm{P}$ & 1.895 & 0.528 & 0.789 & 50.7 & 0 & 1.911 & 0.792 & 50.8 & 1.965 & 0.790 & 50.6 \\
\hline 20528 & 28 USGS & L22 & L531 & $\mathrm{L}$ & $\mathrm{N}$ & 1.928 & 0.519 & 0.794 & 51.0 & 0 & & & & & & \\
\hline 20528 & 28 USGS & L22 & L531 & $\mathrm{T}$ & $\mathrm{P}$ & 1.981 & 0.505 & 0.781 & 49.8 & 0 & 2.018 & 0.789 & 50.4 & & & \\
\hline 20528 & 28 USGS & L22 & L531 & $\mathrm{T}$ & $\mathrm{N}$ & 2.056 & 0.487 & 0.797 & 51.1 & 0 & & & & & & \\
\hline 20314 & 29PSCL & L22 & L746 & V & $\mathrm{P}$ & 1.974 & 0.507 & 0.716 & 91.3 & 2 & 1.976 & 0.715 & 91.0 & 1.976 & 0.715 & 91.0 \\
\hline 20314 & 29PSCL & L22 & L746 & V & $\mathrm{N}$ & 1.977 & 0.506 & 0.714 & 90.7 & 1 & & & & & & \\
\hline 20314 & $29 \mathrm{PSCL}$ & L22 & L746 & $\mathrm{L}$ & $\mathrm{P}$ & 2.914 & 0.343 & 0.512 & 88.6 & 0 & 2.944 & 0.521 & 90.5 & 2.944 & 0.521 & 90.5 \\
\hline 20314 & $29 \mathrm{PSCL}$ & L22 & L746 & $\mathrm{L}$ & $\mathrm{N}$ & 2.974 & 0.336 & 0.530 & 92.4 & 0 & & & & & & \\
\hline 20314 & $29 \mathrm{PSCL}$ & L22 & L746 & $\mathrm{T}$ & $\mathrm{P}$ & 60.241 & 0.017 & 0.109 & 97.9 dead & & 50.120 & 0.067 & 79.2 & & & \\
\hline 20314 & 29PSCL & L22 & L746 & $\mathrm{T}$ & $\mathrm{N}$ & 40.000 & 0.025 & 0.025 & 60.6 dead & & & & & & & \\
\hline 20528 & 30 USGS & L22 & L538 & V & $\mathrm{P}$ & 2.238 & 0.447 & 0.786 & 50.7 & 0 & 2.232 & 0.789 & 50.6 & 2.232 & 0.789 & 50.6 \\
\hline 20528 & 30 USGS & L22 & L538 & V & $\mathrm{N}$ & 2.226 & 0.449 & 0.793 & 50.5 & 0 & & & & & & \\
\hline 20528 & 30 USGS & L22 & L538 & $\mathrm{L}$ & $\mathrm{P}$ & 2.591 & 0.386 & 0.703 & 51.4 & 0 & 2.561 & 0.701 & 51.3 & 2.283 & 0.716 & 46.0 \\
\hline 20528 & 30 USGS & L22 & L538 & $\mathrm{L}$ & $\mathrm{N}$ & 2.531 & 0.395 & 0.699 & 51.2 & 0 & & & & & & \\
\hline 20528 & 30 USGS & L22 & L538 & $\mathrm{T}$ & $\mathrm{P}$ & 2.005 & 0.499 & 0.730 & 40.8 & 0 & 2.005 & 0.730 & 40.8 & & & \\
\hline 20528 & 30 USGS & L22 & L538 & $\mathrm{T}$ & $\mathrm{N}$ & 47.393 & 0.021 & 1.171 & $230.2 \mathrm{bad}$ & & & & & & & \\
\hline 20528 & 31 USGS & L22 & L535 & V & $\mathrm{P}$ & 1.920 & 0.521 & 0.825 & 50.0 & 0 & 1.938 & 0.823 & 50.2 & 1.938 & 0.823 & 50.2 \\
\hline 20528 & 31 USGS & L22 & L535 & V & $\mathrm{N}$ & 1.957 & 0.511 & 0.821 & 50.5 & 0 & & & & & & \\
\hline 20528 & 31 USGS & L22 & L535 & $\mathrm{L}$ & $\mathrm{P}$ & 2.024 & 0.494 & 0.778 & 50.5 & 0 & 1.994 & 0.782 & 50.6 & 2.004 & 0.788 & 50.3 \\
\hline 20528 & 31 USGS & L22 & L535 & $\mathrm{L}$ & $\mathrm{N}$ & 1.964 & 0.509 & 0.786 & 50.7 & 0 & & & & & & \\
\hline 20528 & 31 USGS & L22 & L535 & $\mathrm{T}$ & $\mathrm{P}$ & 2.025 & 0.494 & 0.794 & 49.8 & 0 & 2.014 & 0.795 & 50.1 & & & \\
\hline 20528 & 31 USGS & L22 & L535 & $\mathrm{T}$ & $\mathrm{N}$ & 2.003 & 0.499 & 0.795 & 50.3 & 0 & & & & & & \\
\hline
\end{tabular}




\begin{tabular}{|c|c|c|c|c|c|c|c|c|c|c|c|c|c|c|c|c|}
\hline 20314 & $32 \mathrm{PSCL}$ & L22 & L724 & $\mathrm{V}$ & $\mathrm{P}$ & 1.860 & 0.538 & 0.715 & 88.2 & 2 & 1.874 & 0.727 & 89.0 & 1.874 & 0.727 & 89.0 \\
\hline 20314 & $32 \mathrm{PSCL}$ & L22 & L724 & V & $\mathrm{N}$ & 1.889 & 0.530 & 0.740 & 89.8 & 1 & & & & & & \\
\hline 20314 & $32 \mathrm{PSCL}$ & L22 & L724 & $\mathrm{L}$ & $\mathrm{P}$ & 2.424 & 0.413 & 0.634 & 90.8noisy & & 13.005 & 0.762 & 218.9 & & & \\
\hline 20314 & $32 \mathrm{PSCL}$ & L22 & L724 & $\mathrm{L}$ & $\mathrm{N}$ & 23.585 & 0.042 & 0.889 & 347.1 dead & & & & & & & \\
\hline 20314 & $32 \mathrm{PSCL}$ & L22 & L724 & $\mathrm{T}$ & $\mathrm{P}$ & 23.585 & 0.042 & 0.699 & 295.6 dead & & 13.120 & 0.610 & 192.1 & & & \\
\hline 20314 & $32 \mathrm{PSCL}$ & L22 & L724 & $\mathrm{T}$ & $\mathrm{N}$ & 2.655 & 0.377 & 0.522 & 88.6noisy & & & & & & & \\
\hline 20314 & $33 \mathrm{PSCL}$ & L22 & L448 & V & $P$ & 2.077 & 0.482 & 0.658 & 86.1 & 0 & 2.066 & 0.660 & 86.5 & 2.066 & 0.660 & 86.5 \\
\hline 20314 & $33 \mathrm{PSCL}$ & L22 & L448 & V & $\mathrm{N}$ & 2.055 & 0.487 & 0.663 & 86.9 & 0 & & & & & & \\
\hline 20314 & $33 \mathrm{PSCL}$ & L22 & L448 & $\mathrm{L}$ & $\mathrm{P}$ & 2.455 & 0.407 & 0.578 & 83.7 & 0 & 2.439 & 0.581 & 84.3 & 2.518 & 0.557 & 83.9 \\
\hline 20314 & $33 \mathrm{PSCL}$ & L22 & L448 & $\mathrm{L}$ & $\mathrm{N}$ & 2.422 & 0.413 & 0.583 & 84.9 & 0 & & & & & & \\
\hline 20314 & $33 \mathrm{PSCL}$ & L22 & L448 & $\mathrm{T}$ & $\mathrm{P}$ & 2.591 & 0.386 & 0.527 & 81.9 & 0 & 2.596 & 0.534 & 83.4 & & & \\
\hline 20314 & $33 \mathrm{PSCL}$ & L22 & L448 & $\mathrm{T}$ & $N$ & 2.601 & 0.384 & 0.542 & 84.9 & 0 & & & & & & \\
\hline 20314 & $34 \mathrm{PSCL}$ & L22 & L967 & V & $P$ & 2.097 & 0.477 & 0.768 & 95.1 & 0 & 2.104 & 0.778 & 95.3 & 2.104 & 0.778 & 95.3 \\
\hline 20314 & $34 \mathrm{PSCL}$ & L22 & L967 & V & $\mathrm{N}$ & 2.110 & 0.474 & 0.788 & 95.6 & 0 & & & & & & \\
\hline 20314 & $34 \mathrm{PSCL}$ & L22 & L967 & $\mathrm{L}$ & $P$ & 2.438 & 0.410 & 0.678 & 91.9 & 0 & 2.424 & 0.668 & 92.3 & 2.399 & 0.663 & 90.9 \\
\hline 20314 & $34 \mathrm{PSCL}$ & L22 & L967 & $\mathrm{L}$ & $\mathrm{N}$ & 2.409 & 0.415 & 0.657 & 92.7 & 0 & & & & & & \\
\hline 20314 & $34 \mathrm{PSCL}$ & L22 & L967 & $\mathrm{T}$ & $P$ & 2.402 & 0.416 & 0.668 & 89.7 & 0 & 2.374 & 0.657 & 89.6 & & & \\
\hline 20314 & $34 \mathrm{PSCL}$ & L22 & L967 & $\mathrm{T}$ & $\mathrm{N}$ & 2.347 & 0.426 & 0.646 & 89.4 & 0 & & & & & & \\
\hline 20314 & $35 \mathrm{PSCL}$ & L22 & L953 & V & $P$ & 2.023 & 0.494 & 0.736 & 90.7 & 0 & 2.053 & 0.760 & 92.9 & 2.053 & 0.760 & 92.9 \\
\hline 20314 & $35 \mathrm{PSCL}$ & L22 & L953 & $\mathrm{V}$ & $\mathrm{N}$ & 2.082 & 0.480 & 0.784 & 95.1 & 0 & & & & & & \\
\hline 20314 & $35 \mathrm{PSCL}$ & L22 & L953 & $\mathrm{L}$ & $\mathrm{P}$ & 1.816 & 0.551 & 0.785 & 87.9 & 0 & 1.830 & 0.786 & 88.1 & 1.881 & 0.770 & 88.2 \\
\hline 20314 & $35 \mathrm{PSCL}$ & L22 & L953 & $\mathrm{L}$ & $\mathrm{N}$ & 1.844 & 0.542 & 0.787 & 88.2 & 0 & & & & & & \\
\hline 20314 & $35 \mathrm{PSCL}$ & L22 & L953 & $\mathrm{T}$ & $\mathrm{P}$ & 1.895 & 0.528 & 0.761 & 88.7 & 0 & 1.932 & 0.755 & 88.4 & & & \\
\hline 20314 & $35 \mathrm{PSCL}$ & L22 & L953 & $\mathrm{T}$ & $\mathrm{N}$ & 1.970 & 0.508 & 0.749 & 88.1 & 0 & & & & & & \\
\hline 20314 & $36 \mathrm{PSCL}$ & L22 & L458 & V & $\mathrm{P}$ & 2.178 & 0.459 & 0.621 & 84.6 & 0 & 2.147 & 0.619 & 84.5 & 2.147 & 0.619 & 84.5 \\
\hline 20314 & $36 \mathrm{PSCL}$ & L22 & L458 & V & $\mathrm{N}$ & 2.116 & 0.473 & 0.617 & 84.5 & 0 & & & & & & \\
\hline 20314 & $36 \mathrm{PSCL}$ & L22 & L458 & $\mathrm{L}$ & $\mathrm{P}$ & 2.030 & 0.493 & 0.748 & 90.3 & 0 & 1.996 & 0.753 & 90.8 & 2.204 & 0.672 & 88.6 \\
\hline 20314 & $36 \mathrm{PSCL}$ & L22 & L458 & $\mathrm{L}$ & $\mathrm{N}$ & 1.962 & 0.510 & 0.757 & 91.3 & 0 & & & & & & \\
\hline 20314 & $36 \mathrm{PSCL}$ & L22 & L458 & $\mathrm{T}$ & $\mathrm{P}$ & 2.422 & 0.413 & 0.585 & 85.7 & 0 & 2.412 & 0.590 & 86.4 & & & \\
\hline 20314 & $36 \mathrm{PSCL}$ & L22 & L458 & $\mathrm{T}$ & $\mathrm{N}$ & 2.402 & 0.416 & 0.596 & 87.1 & 0 & & & & & & \\
\hline
\end{tabular}




\begin{tabular}{|c|c|c|c|c|c|c|c|c|c|c|c|c|c|c|c|c|}
\hline 20528 & 37PSCL & L22 & L462 & V & $P$ & 2.046 & 0.489 & 0.732 & 93.6 & 2 & 2.019 & 0.733 & 93.4 & 2.019 & 0.733 & 93.4 \\
\hline 20528 & 37PSCL & L22 & L462 & V & $\mathrm{N}$ & 1.992 & 0.502 & 0.734 & 93.2 & 2 & & & & & & \\
\hline 20528 & 37PSCL & L22 & L462 & $\mathrm{L}$ & $\mathrm{P}$ & 2.192 & 0.456 & 0.746 & 94.1 & 0 & 2.124 & 0.756 & 94.2 & 2.055 & 0.755 & 93.4 \\
\hline 20528 & 37PSCL & L22 & L462 & $\mathrm{L}$ & $\mathrm{N}$ & 2.056 & 0.486 & 0.765 & 94.3 & 1 & & & & & & \\
\hline 20528 & 37PSCL & L22 & L462 & $\mathrm{T}$ & $\mathrm{P}$ & 2.008 & 0.498 & 0.752 & 92.6 & 0 & 1.986 & 0.754 & 92.6 & & & \\
\hline 20528 & 37PSCL & L22 & L462 & $\mathrm{T}$ & $\mathrm{N}$ & 1.965 & 0.509 & 0.755 & 92.6 & 1 & & & & & & \\
\hline 20314 & 38PSCL & L22 & L507 & V & $\mathrm{P}$ & 2.251 & 0.444 & 0.749 & 93.4 & 0 & 2.212 & 0.739 & 92.7 & 2.212 & 0.739 & 92.7 \\
\hline 20314 & 38PSCL & L22 & L507 & V & $\mathrm{N}$ & 2.173 & 0.460 & 0.729 & 92.0 & 0 & & & & & & \\
\hline 20314 & $38 \mathrm{PSCL}$ & L22 & L507 & $\mathrm{L}$ & $\mathrm{P}$ & 2.007 & 0.498 & 0.855 & 95.1 & 0 & 1.861 & 0.834 & 91.6 & 2.089 & 0.727 & 89.2 \\
\hline 20314 & 38PSCL & L22 & L507 & $\mathrm{L}$ & $\mathrm{N}$ & 1.715 & 0.583 & 0.812 & 88.2 & 1 & & & & & & \\
\hline 20314 & $38 \mathrm{PSCL}$ & L22 & L507 & $\mathrm{T}$ & $\mathrm{P}$ & 2.329 & 0.429 & 0.610 & 85.2 & 0 & 2.316 & 0.620 & 86.7 & & & \\
\hline 20314 & $38 \mathrm{PSCL}$ & L22 & L507 & $\mathrm{T}$ & $\mathrm{N}$ & 2.303 & 0.434 & 0.631 & 88.2 & 0 & & & & & & \\
\hline 20402 & $39 \mathrm{PSCL}$ & L22 & L742 & V & $\mathrm{P}$ & 1.925 & 0.520 & 0.739 & 90.8 & 1 & 1.926 & 0.743 & 91.3 & 1.926 & 0.743 & \\
\hline 20402 & 39PSCL & L22 & L742 & V & $\mathrm{N}$ & 1.926 & 0.519 & 0.747 & 91.7 & 1 & & & & & & \\
\hline 20402 & 39PSCL & L22 & L742 & $\mathrm{L}$ & $\mathrm{P}$ & 2.217 & 0.451 & 0.608 & 85.3 & 0 & 2.148 & 0.609 & 85.7 & 2.000 & 0.672 & 87.5 \\
\hline 20402 & 39PSCL & L22 & L742 & $\mathrm{L}$ & $\mathrm{N}$ & 2.079 & 0.481 & 0.611 & 86.1 & 0 & & & & & & \\
\hline 20402 & 39PSCL & L22 & L742 & $\mathrm{T}$ & $\mathrm{P}$ & 1.891 & 0.529 & 0.746 & 90.3 & 2 & 1.853 & 0.735 & 89.3 & & & \\
\hline 20402 & $39 \mathrm{PSCL}$ & L22 & L742 & $\mathrm{T}$ & $\mathrm{N}$ & 1.816 & 0.551 & 0.725 & 88.2 & 2 & & & & & & \\
\hline 20418 & 40PSCL & L22 & L491 & V & $\mathrm{P}$ & 2.041 & 0.490 & 0.771 & 93.3 & 0 & 2.037 & 0.768 & 92.8 & 2.037 & 0.768 & 92.8 \\
\hline 20418 & 40 PSCL & L22 & L491 & V & $\mathrm{N}$ & 2.033 & 0.492 & 0.766 & 92.3 & 0 & & & & & & \\
\hline 20418 & 40PSCL & L22 & L491 & L & $\mathrm{P}$ & 2.015 & 0.496 & 0.762 & 87.2 & 0 & 1.937 & 0.760 & 86.0 & 2.053 & 0.729 & 88.2 \\
\hline 20418 & 40 PSCL & L22 & L491 & $\mathrm{L}$ & $\mathrm{N}$ & 1.860 & 0.538 & 0.757 & 84.9 & 0 & & & & & & \\
\hline 20418 & 40 PSCL & L22 & L491 & $\mathrm{T}$ & $\mathrm{P}$ & 2.288 & 0.437 & 0.716 & 92.5 & 0 & 2.169 & 0.698 & 90.3 & & & \\
\hline 20418 & 40 PSCL & L22 & L491 & $\mathrm{T}$ & $\mathrm{N}$ & 2.050 & 0.488 & 0.679 & 88.1 & 0 & & & & & & \\
\hline 20402 & $41 \mathrm{PSCL}$ & L22 & L747 & V & $\mathrm{P}$ & 1.934 & 0.517 & 0.635 & 50.3 & 1 & 2.015 & 0.709 & 73.0 & 2.015 & 0.709 & 73.0 \\
\hline 20402 & $41 \mathrm{PSCL}$ & L22 & L747 & V & $\mathrm{N}$ & 2.096 & 0.477 & 0.782 & 95.7 & 1 & & & & & & \\
\hline 20402 & $41 \mathrm{PSCL}$ & L22 & L747 & $\mathrm{L}$ & $\mathrm{P}$ & 2.151 & 0.465 & 0.832 & 99.8 & 1 & 2.015 & 0.820 & 96.2 & 1.917 & 0.812 & 93.2 \\
\hline 20402 & $41 \mathrm{PSCL}$ & L22 & L747 & $\mathrm{L}$ & $\mathrm{N}$ & 1.880 & 0.532 & 0.808 & 92.5 & 2 & & & & & & \\
\hline 20402 & $41 \mathrm{PSCL}$ & L22 & L747 & $\mathrm{T}$ & $\mathrm{P}$ & 1.748 & 0.572 & 0.824 & 91.2 & 0 & 1.819 & 0.804 & 90.2 & & & \\
\hline 20402 & $41 \mathrm{PSCL}$ & L22 & L747 & $\mathrm{T}$ & $\mathrm{N}$ & 1.890 & 0.529 & 0.784 & 89.2 & 0 & & & & & & \\
\hline
\end{tabular}




\begin{tabular}{|c|c|c|c|c|c|c|c|c|c|}
\hline 20402 & $41 \mathrm{PSCL}$ & L22 & L747 & $\mathrm{V}$ & $P$ & 2.101 & 0.476 & 0.781 & 95.9 \\
\hline 20402 & 41 PSCL & L22 & L747 & V & $\mathrm{N}$ & 2.141 & 0.467 & 0.806 & 98.5 \\
\hline 20402 & $41 \mathrm{PSCL}$ & L22 & L747 & $\mathrm{L}$ & $P$ & 1.859 & 0.538 & 0.795 & 91.3 \\
\hline 20402 & $41 \mathrm{PSCL}$ & L22 & L747 & $\mathrm{L}$ & $\mathrm{N}$ & 1.715 & 0.583 & 0.806 & 89.4 \\
\hline 20402 & $41 \mathrm{PSCL}$ & L22 & L747 & $\mathrm{T}$ & $P$ & 1.887 & 0.530 & 0.795 & 89.5 \\
\hline 20402 & $41 \mathrm{PSCL}$ & L22 & L747 & $\mathrm{T}$ & $\mathrm{N}$ & 1.912 & 0.523 & 0.772 & 88.6 \\
\hline 20402 & 42PSCL & L22 & L643 & V & $P$ & 2.160 & 0.463 & 0.768 & 96.6 \\
\hline 20402 & 42PSCL & L22 & L643 & V & $\mathrm{N}$ & 2.101 & 0.476 & 0.771 & 96.2 \\
\hline 20402 & $42 \mathrm{PSCL}$ & L22 & L643 & $\mathrm{L}$ & $\mathrm{P}$ & 2.564 & 0.390 & 0.657 & 96.0 \\
\hline 20402 & $42 \mathrm{PSCL}$ & L22 & L643 & $\mathrm{L}$ & $\mathrm{N}$ & 2.427 & 0.412 & 0.660 & 95.5 \\
\hline 20402 & 42PSCL & L22 & L643 & $\mathrm{T}$ & $\mathrm{P}$ & 1.618 & 0.618 & 0.806 & 84.6 \\
\hline 20402 & $42 \mathrm{PSCL}$ & L22 & L643 & $\mathrm{T}$ & $\mathrm{N}$ & 1.984 & 0.504 & 0.777 & 87.6 \\
\hline 20402 & $42 \mathrm{PSCL}$ & L22 & L643 & V & $\mathrm{P}$ & 2.151 & 0.465 & 0.748 & 95.4 \\
\hline 20402 & $42 \mathrm{PSCL}$ & L22 & L643 & V & $\mathrm{N}$ & 2.066 & 0.484 & 0.747 & 94.2 \\
\hline 20402 & 42PSCL & L22 & L643 & $\mathrm{L}$ & $\mathrm{P}$ & 2.500 & 0.400 & 0.642 & 93.7 \\
\hline 20402 & 42 PSCL & L22 & L643 & $\mathrm{L}$ & $\mathrm{N}$ & 2.469 & 0.405 & 0.690 & 97.6 \\
\hline 20402 & $42 \mathrm{PSCL}$ & L22 & L643 & $\mathrm{T}$ & $\mathrm{P}$ & 1.656 & 0.604 & 0.826 & 86.3 \\
\hline 20402 & $42 \mathrm{PSCL}$ & L22 & L643 & $\mathrm{T}$ & $\mathrm{N}$ & 2.016 & 0.496 & 0.791 & 89.1 \\
\hline 20402 & 43PSCL & L22 & L463 & V & $\mathrm{P}$ & 1.913 & 0.523 & 0.799 & 93.5 \\
\hline 20402 & 43PSCL & L22 & L463 & $\mathrm{V}$ & $\mathrm{N}$ & 1.904 & 0.525 & 0.791 & 92.7 \\
\hline 20402 & 43PSCL & L22 & L463 & $\mathrm{L}$ & $\mathrm{P}$ & 2.258 & 0.443 & 0.626 & 84.2 \\
\hline 20402 & 43 PSCL & L22 & L463 & $\mathrm{L}$ & $\mathrm{N}$ & 2.133 & 0.469 & 0.635 & 85.6 \\
\hline 20402 & $43 \mathrm{PSCL}$ & L22 & L463 & $\mathrm{T}$ & $P$ & 1.924 & 0.520 & 0.804 & 92.3 \\
\hline 20402 & $43 \mathrm{PSCL}$ & L22 & L463 & $\mathrm{T}$ & $N$ & 1.916 & 0.522 & 0.832 & 93.6 \\
\hline 20402 & 44PSCL & L22 & L1486 & V & $\mathrm{P}$ & 2.013 & 0.497 & 0.739 & 92.5 \\
\hline 20402 & $44 \mathrm{PSCL}$ & L22 & L1486 & V & $\mathrm{N}$ & 1.974 & 0.507 & 0.739 & 92.1 \\
\hline 20402 & 44PSCL & L22 & L1486 & $\mathrm{L}$ & $\mathrm{P}$ & 1.955 & 0.512 & 0.756 & 91.8 \\
\hline 20402 & 44PSCL & L22 & L1486 & $\mathrm{L}$ & $\mathrm{N}$ & 1.910 & 0.524 & 0.780 & 92.0 \\
\hline 20402 & 44PSCL & L22 & L1486 & $\mathrm{T}$ & $\mathrm{P}$ & 1.595 & 0.627 & 0.776 & 84.7 \\
\hline 20402 & $44 \mathrm{PSCL}$ & L22 & L1486 & $\mathrm{T}$ & $N$ & 1.727 & 0.579 & 0.780 & 87.5 \\
\hline
\end{tabular}






\begin{tabular}{|c|c|c|c|c|c|c|c|c|c|c|c|c|c|c|c|c|}
\hline 20402 & 45PSCL & L22 & L640 & $\mathrm{V}$ & $P$ & 2.007 & 0.498 & 0.797 & 95.8 & 2 & 2.009 & 0.793 & 95.5 & 2.009 & 0.793 & 95.5 \\
\hline 20402 & 45PSCL & L22 & L640 & V & $\mathrm{N}$ & 2.011 & 0.497 & 0.788 & 95.1 & 2 & & & & & & \\
\hline 20402 & 45PSCL & L22 & L640 & $\mathrm{L}$ & $\mathrm{P}$ & 2.101 & 0.476 & 0.767 & 94.8 & 1 & 1.978 & 0.760 & 92.6 & 1.886 & 0.809 & 92.9 \\
\hline 20402 & 45PSCL & L22 & L640 & $\mathrm{L}$ & $\mathrm{N}$ & 1.855 & 0.539 & 0.753 & 90.5 & 2 & & & & & & \\
\hline 20402 & 45PSCL & L22 & L640 & $\mathrm{T}$ & $P$ & 1.845 & 0.542 & 0.860 & 94.3 & 2 & 1.794 & 0.859 & 93.1 & & & \\
\hline 20402 & $45 \mathrm{PSCL}$ & L22 & L640 & $\mathrm{T}$ & $\mathrm{N}$ & 1.742 & 0.574 & 0.858 & 91.9 & 2 & & & & & & \\
\hline 20227 & 46PSCL & L22 & L949 & $\mathrm{V}$ & $P$ & 2.078 & 0.481 & 0.749 & 93.4 & 0 & 2.070 & 0.752 & 93.4 & 2.070 & 0.752 & 93.4 \\
\hline 20227 & $46 \mathrm{PSCL}$ & L22 & L949 & V & $\mathrm{N}$ & 2.063 & 0.485 & 0.755 & 93.5 & 0 & & & & & & \\
\hline 20227 & $46 \mathrm{PSCL}$ & L22 & L949 & $\mathrm{L}$ & $\mathrm{P}$ & 2.035 & 0.491 & 0.763 & 92.3 & 0 & 2.014 & 0.752 & 91.1 & 1.952 & 0.764 & 89.6 \\
\hline 20227 & 46PSCL & L22 & L949 & $\mathrm{L}$ & $\mathrm{N}$ & 1.992 & 0.502 & 0.741 & 90.0 & 0 & & & & & & \\
\hline 20227 & $46 \mathrm{PSCL}$ & L22 & L949 & $\mathrm{T}$ & $\mathrm{P}$ & 1.820 & 0.549 & 0.784 & 88.5 & 0 & 1.891 & 0.775 & 88.1 & & & \\
\hline 20227 & $46 \mathrm{PSCL}$ & L22 & L949 & $\mathrm{T}$ & $\mathrm{N}$ & 1.961 & 0.510 & 0.766 & 87.8 & 0 & & & & & & \\
\hline 20314 & $50 \mathrm{PSCL}$ & L22 & L1508 & V & $\mathrm{P}$ & 2.172 & 0.461 & 0.767 & 97.0 & 1 & 2.160 & 0.771 & 97.4 & 2.160 & 0.771 & 97.4 \\
\hline 20314 & $50 \mathrm{PSCL}$ & L22 & L1508 & $\mathrm{V}$ & $\mathrm{N}$ & 2.148 & 0.466 & 0.776 & 97.7 & 1 & & & & & & \\
\hline 20314 & $50 \mathrm{PSCL}$ & L22 & L1508 & $\mathrm{L}$ & $\mathrm{P}$ & 2.277 & 0.439 & 0.663 & 88.2 & 0 & 2.188 & 0.652 & 87.0 & 2.167 & 0.694 & 90.0 \\
\hline 20314 & $50 \mathrm{PSCL}$ & L22 & L1508 & $\mathrm{L}$ & $\mathrm{N}$ & 2.099 & 0.476 & 0.641 & 85.8 & 0 & & & & & & \\
\hline 20314 & $50 \mathrm{PSCL}$ & L22 & L1508 & $\mathrm{T}$ & $\mathrm{P}$ & 2.226 & 0.449 & 0.755 & 95.0 & 0 & 2.146 & 0.736 & 93.0 & & & \\
\hline 20314 & $50 \mathrm{PSCL}$ & L22 & L1508 & $\mathrm{T}$ & $\mathrm{N}$ & 2.066 & 0.484 & 0.717 & 91.1 & 0 & & & & & & \\
\hline 20528 & 51 USGS & L22 & L537 & V & $\mathrm{P}$ & 1.993 & 0.502 & 0.840 & 51.5 & 0 & 2.000 & 0.845 & 51.6 & 2.000 & 0.845 & 51.6 \\
\hline 20528 & 51 USGS & L22 & L537 & V & $\mathrm{N}$ & 2.007 & 0.498 & 0.850 & 51.8 & 0 & & & & & & \\
\hline 20528 & 51 USGS & L22 & L537 & $\mathrm{L}$ & $\mathrm{P}$ & 2.093 & 0.478 & 0.773 & 50.1 & 0 & 2.073 & 0.772 & 50.0 & 1.915 & 0.782 & 50.2 \\
\hline 20528 & 51 USGS & L22 & L537 & $\mathrm{L}$ & $\mathrm{N}$ & 2.053 & 0.487 & 0.772 & 49.8 & 0 & & & & & & \\
\hline 20528 & 51 USGS & L22 & L537 & $\mathrm{T}$ & $\mathrm{P}$ & 1.700 & 0.588 & 0.795 & 50.5 & 0 & 1.757 & 0.791 & 0.5 & & & \\
\hline 20528 & 51 USGS & L22 & L537 & $\mathrm{T}$ & $\mathrm{N}$ & 1.814 & 0.551 & 0.787 & 50.5 & 0 & & & & & & \\
\hline 20314 & $52 \mathrm{PSCL}$ & L22 & L957 & V & $\mathrm{P}$ & 2.070 & 0.483 & 0.719 & 89.6 & 0 & 2.112 & 0.746 & 91.9 & 2.112 & 0.746 & 91.9 \\
\hline 20314 & $52 \mathrm{PSCL}$ & L22 & L957 & V & $\mathrm{N}$ & 2.154 & 0.464 & 0.772 & 94.2 & 0 & & & & & & \\
\hline 20314 & $52 \mathrm{PSCL}$ & L22 & L957 & $\mathrm{L}$ & $\mathrm{P}$ & 2.060 & 0.486 & 0.753 & 93.8 & 0 & 2.091 & 0.741 & 93.2 & 1.973 & 0.780 & 92.3 \\
\hline 20314 & $52 \mathrm{PSCL}$ & L22 & L957 & $\mathrm{L}$ & $\mathrm{N}$ & 2.121 & 0.471 & 0.729 & 92.6 & 0 & & & & & & \\
\hline 20314 & $52 \mathrm{PSCL}$ & L22 & L957 & $\mathrm{T}$ & $\mathrm{P}$ & 1.842 & 0.543 & 0.815 & 91.2 & 1 & 1.856 & 0.819 & 91.4 & & & \\
\hline 20314 & $52 \mathrm{PSCL}$ & L22 & L957 & $\mathrm{T}$ & $\mathrm{N}$ & 1.871 & 0.535 & 0.824 & 91.6 & 0 & & & & & & \\
\hline
\end{tabular}




\begin{tabular}{|c|c|c|c|c|c|c|c|c|c|c|c|c|c|c|c|c|}
\hline 20528 & $53 \mathrm{PSCL}$ & L22 & L968 & V & $\mathrm{P}$ & 2.074 & 0.482 & 0.745 & 92.5 & 0 & 2.069 & 0.749 & 92.0 & 2.069 & 0.749 & 92.0 \\
\hline 20528 & 53PSCL & L22 & L968 & V & $\mathrm{N}$ & 2.063 & 0.485 & 0.752 & 91.6 & 0 & & & & & & \\
\hline 20528 & $53 \mathrm{PSCL}$ & L22 & L968 & $\mathrm{L}$ & $\mathrm{P}$ & 2.186 & 0.457 & 0.835 & 99.1 & 0 & 2.192 & 0.828 & 98.5 & 1.827 & 0.918 & 50 \\
\hline 20528 & 53PSCL & L22 & L968 & $\mathrm{L}$ & $\mathrm{N}$ & 2.198 & 0.455 & 0.822 & 97.9 & 0 & & & & & & \\
\hline 20528 & $53 \mathrm{PSCL}$ & L22 & L968 & $\mathrm{T}$ & $\mathrm{P}$ & 1.332 & 0.751 & 1.015 & 84.7 & 2 & 1.463 & 1.008 & 87.6 & & & \\
\hline 20528 & $53 \mathrm{PSCL}$ & L22 & L968 & $\mathrm{T}$ & $\mathrm{N}$ & 1.593 & 0.628 & 1.002 & 90.6 & 2 & & & & & & \\
\hline 20418 & 54 USGS & L22 & L523 & V & $P$ & 2.253 & 0.444 & 0.769 & 51.5 & 0 & 2.285 & 0.782 & 52.2 & 2.285 & 0.782 & 52.2 \\
\hline 20418 & 54 USGS & L22 & L523 & V & $\mathrm{N}$ & 2.316 & 0.432 & 0.794 & 52.9 & 0 & & & & & & \\
\hline 20418 & 54 USGS & L22 & L523 & $\mathrm{L}$ & $\mathrm{P}$ & 2.028 & 0.493 & 0.771 & 50.9 & 0 & 2.038 & 0.770 & 50.8 & 2.061 & 0.787 & 51.0 \\
\hline 20418 & 54 USGS & L22 & L523 & $\mathrm{L}$ & $\mathrm{N}$ & 2.048 & 0.488 & 0.768 & 50.8 & 0 & & & & & & \\
\hline 20418 & 54 USGS & L22 & L523 & $\mathrm{T}$ & $\mathrm{P}$ & 2.055 & 0.487 & 0.789 & 50.4 & 0 & 2.083 & 0.804 & 51.2 & & & \\
\hline 20418 & 54 USGS & L22 & L523 & $\mathrm{T}$ & $\mathrm{N}$ & 2.112 & 0.474 & 0.820 & 52.0 & 0 & & & & & & \\
\hline 20528 & 56 USGS & L22 & L536 & V & $P$ & 1.958 & 0.511 & 0.799 & 51.1 & 0 & 1.962 & 0.803 & 51.2 & 1.962 & 0.803 & 51.2 \\
\hline 20528 & 56 USGS & L22 & L536 & V & $\mathrm{N}$ & 1.966 & 0.509 & 0.808 & 51.4 & 0 & & & & & & \\
\hline 20528 & 56 USGS & L22 & L536 & $\mathrm{L}$ & $\mathrm{P}$ & 1.825 & 0.548 & 0.834 & 49.4 & 0 & 1.819 & 0.834 & 49.5 & 1.916 & 0.798 & 49.6 \\
\hline 20528 & 56 USGS & L22 & L536 & $\mathrm{L}$ & $\mathrm{N}$ & 1.813 & 0.552 & 0.835 & 49.5 & 0 & & & & & & \\
\hline 20528 & 56 USGS & L22 & L536 & $\mathrm{T}$ & $\mathrm{P}$ & 2.031 & 0.492 & 0.770 & 50.1 & 0 & 2.012 & 0.762 & 49.7 & & & \\
\hline 20528 & 56 USGS & L22 & L536 & $\mathrm{T}$ & $\mathrm{N}$ & 1.993 & 0.502 & 0.755 & 49.3 & 0 & & & & & & \\
\hline 20314 & $57 \mathrm{PSCL}$ & L22 & L951 & V & $\mathrm{P}$ & 2.061 & 0.485 & 0.770 & 92.3 & 0 & 2.037 & 0.754 & 91.0 & 2.037 & 0.754 & 91.0 \\
\hline 20314 & $57 \mathrm{PSCL}$ & L22 & L951 & V & $\mathrm{N}$ & 2.013 & 0.497 & 0.738 & 89.7 & 0 & & & & & & \\
\hline 20314 & 57PSCL & L22 & L951 & $\mathrm{L}$ & $\mathrm{P}$ & 2.508 & 0.399 & 0.533 & 84.2 & 0 & 2.492 & 0.547 & 85.7 & 2.189 & 0.656 & 86.2 \\
\hline 20314 & $57 \mathrm{PSCL}$ & L22 & L951 & $\mathrm{L}$ & $\mathrm{N}$ & 2.477 & 0.404 & 0.560 & 87.1 & 0 & & & & & & \\
\hline 20314 & 57PSCL & L22 & L951 & $\mathrm{T}$ & $\mathrm{P}$ & 1.960 & 0.510 & 0.758 & 86.4 & 0 & 1.885 & 0.766 & 86.8 & & & \\
\hline 20314 & 57PSCL & L22 & L951 & $\mathrm{T}$ & $\mathrm{N}$ & 1.811 & 0.552 & 0.773 & 87.1 & 0 & & & & & & \\
\hline 20314 & 58PSCL & L22 & L493 & V & $P$ & 2.107 & 0.475 & 0.890 & 83.6 & 0 & 2.123 & 0.899 & 83.6 & 2.123 & 0.899 & 83.6 \\
\hline 20314 & 58PSCL & L22 & L493 & V & $\mathrm{N}$ & 2.139 & 0.468 & 0.907 & 83.7 & 0 & & & & & & \\
\hline 20314 & 58PSCL & L22 & L493 & $\mathrm{L}$ & $\mathrm{P}$ & 1.742 & 0.574 & 0.814 & 90.1 & 1 & 1.806 & 0.792 & 90.0 & 2.270 & 0.661 & 88.1 \\
\hline 20314 & 58PSCL & L22 & L493 & $\mathrm{L}$ & $\mathrm{N}$ & 1.869 & 0.535 & 0.770 & 90.0 & 0 & & & & & & \\
\hline 20314 & 58PSCL & L22 & L493 & $\mathrm{T}$ & $\mathrm{P}$ & 2.765 & 0.362 & 0.527 & 85.6 & 0 & 2.734 & 0.530 & 86.1 & & & \\
\hline 20314 & $58 \mathrm{PSCL}$ & L22 & L493 & $\mathrm{T}$ & $\mathrm{N}$ & 2.703 & 0.370 & 0.532 & 86.7 & 0 & & & & & & \\
\hline
\end{tabular}




\begin{tabular}{|c|c|c|c|c|c|c|c|c|c|c|c|c|c|c|c|c|}
\hline 20427 & 59PSCL & L22 & L528 & V & $\mathrm{P}$ & 2.081 & 0.481 & 0.835 & 51.7 & 0 & 2.083 & 0.825 & 51.6 & 2.083 & 0.825 & 51.6 \\
\hline 20427 & 59PSCL & L22 & L528 & V & $\mathrm{N}$ & 2.085 & 0.480 & 0.815 & 51.5 & 0 & & & & & & \\
\hline 20427 & 59PSCL & L22 & L528 & $\mathrm{L}$ & $\mathrm{P}$ & 2.000 & 0.500 & 0.975 & 66.0 & 0 & 1.964 & 0.959 & 65.3 & 2.071 & 0.901 & 58.5 \\
\hline 20427 & 59PSCL & L22 & L528 & $\mathrm{L}$ & $\mathrm{N}$ & 1.928 & 0.519 & 0.944 & 64.5 & 0 & & & & & & \\
\hline 20427 & 59PSCL & L22 & L528 & $\mathrm{T}$ & $P$ & 2.173 & 0.460 & 0.834 & 51.6 & 0 & 2.178 & 0.842 & 51.8 & & & \\
\hline 20427 & 59PSCL & L22 & L528 & $\mathrm{T}$ & $\mathrm{N}$ & 2.182 & 0.458 & 0.850 & 52.1 & 0 & & & & & & \\
\hline 20314 & 60PSCL & L22 & L733 & V & $P$ & 1.839 & 0.544 & 0.752 & 89.9 & 2 & 1.840 & 0.742 & 89.3 & 1.840 & 0.742 & 89.3 \\
\hline 20314 & 60PSCL & L22 & L733 & V & $\mathrm{N}$ & 1.842 & 0.543 & 0.732 & 88.7 & 2 & & & & & & \\
\hline 20314 & $60 \mathrm{PSCL}$ & L22 & L733 & $\mathrm{L}$ & $P$ & 1.888 & 0.530 & 0.761 & 90.3 & 0 & 1.855 & 0.763 & 89.9 & 2.029 & 0.696 & 90.3 \\
\hline 20314 & $60 \mathrm{PSCL}$ & L22 & L733 & $\mathrm{L}$ & $\mathrm{N}$ & 1.822 & 0.549 & 0.764 & 89.5 & 1 & & & & & & \\
\hline 20314 & $60 \mathrm{PSCL}$ & L22 & L733 & $\mathrm{T}$ & $\mathrm{P}$ & 2.236 & 0.447 & 0.621 & 89.6 & 2 & 2.204 & 0.629 & 90.6 & & & \\
\hline 20314 & 60 PSCL & L22 & L733 & $\mathrm{T}$ & $\mathrm{N}$ & 2.171 & 0.461 & 0.637 & 91.7 & 2 & & & & & & \\
\hline 20212 & 61 PSCL & L22 & L950 & V & $P$ & 2.051 & 0.488 & 0.755 & 91.0 & 0 & 2.057 & 0.762 & 91.5 & 2.057 & 0.762 & 91.5 \\
\hline 20212 & 61 PSCL & L22 & L950 & V & $\mathrm{N}$ & 2.062 & 0.485 & 0.770 & 91.9 & 0 & & & & & & \\
\hline 20212 & 61 PSCL & L22 & L950 & $\mathrm{L}$ & $\mathrm{P}$ & 1.973 & 0.507 & 0.795 & 92.3 & 0 & 1.974 & 0.786 & 92.0 & 2.072 & 0.733 & 90.9 \\
\hline 20212 & 61 PSCL & L22 & L950 & $\mathrm{L}$ & $\mathrm{N}$ & 1.974 & 0.507 & 0.777 & 91.8 & 0 & & & & & & \\
\hline 20212 & 61 PSCL & L22 & L950 & $\mathrm{T}$ & $P$ & 2.161 & 0.463 & 0.663 & 87.9 & 0 & 2.170 & 0.680 & 89.7 & & & \\
\hline 20212 & 61 PSCL & L22 & L950 & $\mathrm{T}$ & $\mathrm{N}$ & 2.180 & 0.459 & 0.697 & 91.6 & 0 & & & & & & \\
\hline 20314 & 62PSCL & L22 & L743 & V & $P$ & 1.988 & 0.503 & 0.727 & 91.2 & 0 & 1.951 & 0.718 & 90.2 & 1.951 & 0.718 & 90.2 \\
\hline 20314 & $62 \mathrm{PSCL}$ & L22 & L743 & V & $\mathrm{N}$ & 1.914 & 0.523 & 0.710 & 89.1 & 1 & & & & & & \\
\hline 20314 & $62 \mathrm{PSCL}$ & L22 & L743 & $\mathrm{L}$ & $\mathrm{P}$ & 1.664 & 0.601 & 0.827 & 88.7 & 2 & 1.821 & 0.840 & 92.1 & 1.805 & 0.817 & 91.0 \\
\hline 20314 & 62PSCL & L22 & L743 & $\mathrm{L}$ & $\mathrm{N}$ & 1.978 & 0.506 & 0.852 & 95.4 & 0 & & & & & & \\
\hline 20314 & $62 \mathrm{PSCL}$ & L22 & L743 & $\mathrm{T}$ & $\mathrm{P}$ & 1.802 & 0.555 & 0.784 & 89.5 & 2 & 1.790 & 0.794 & 90.0 & & & \\
\hline 20314 & $62 \mathrm{PSCL}$ & L22 & L743 & $\mathrm{T}$ & $\mathrm{N}$ & 1.777 & 0.563 & 0.803 & 90.5 & 2 & & & & & & \\
\hline 20222 & 63 USGS & L22 & L539 & V & $P$ & 2.023 & 0.494 & 0.812 & 51.2 & 0 & 2.048 & 0.822 & 51.8 & 2.048 & 0.822 & 51.8 \\
\hline 20222 & 63 USGS & L22 & L539 & V & $\mathrm{N}$ & 2.074 & 0.482 & 0.833 & 52.3 & 0 & & & & & & \\
\hline 20222 & 63 USGS & L22 & L539 & $\mathrm{L}$ & $\mathrm{P}$ & 2.081 & 0.481 & 0.831 & 52.6 & 0 & 2.082 & 0.818 & 52.2 & 2.056 & 0.799 & 50.7 \\
\hline 20222 & 63 USGS & L22 & L539 & $\mathrm{L}$ & $\mathrm{N}$ & 2.082 & 0.480 & 0.806 & 51.9 & 0 & & & & & & \\
\hline 20222 & 63 USGS & L22 & L539 & $\mathrm{T}$ & $\mathrm{P}$ & 2.032 & 0.492 & 0.789 & 49.6 & 0 & 2.030 & 0.780 & 49.3 & & & \\
\hline 20222 & 63 USGS & L22 & L539 & $\mathrm{T}$ & $\mathrm{N}$ & 2.027 & 0.493 & 0.772 & 48.9 & 0 & & & & & & \\
\hline
\end{tabular}




\begin{tabular}{|c|c|c|c|c|c|c|c|c|c|c|c|c|c|c|c|c|}
\hline 20418 & 64PSCL & L22 & L465 & V & $\mathrm{P}$ & 2.304 & 0.434 & 0.667 & 88.5 & 0 & 2.268 & 0.650 & 87.7 & 2.268 & 0.650 & 87.7 \\
\hline 20418 & 64PSCL & L22 & L465 & V & $\mathrm{N}$ & 2.232 & 0.448 & 0.633 & 86.8 & 0 & & & & & & \\
\hline 20418 & 64PSCL & L22 & L465 & $L$ & $\mathrm{P}$ & 1.938 & 0.516 & 0.773 & 90.2 & 0 & 1.928 & 0.787 & 91.3 & 1.928 & 0.802 & 91.6 \\
\hline 20418 & 64PSCL & L22 & L465 & $\mathrm{L}$ & $\mathrm{N}$ & 1.919 & 0.521 & 0.801 & 92.3 & 1 & & & & & & \\
\hline 20418 & 64PSCL & L22 & L465 & $\mathrm{T}$ & $P$ & 1.977 & 0.506 & 0.805 & 91.7 & 0 & 1.927 & 0.817 & 91.9 & & & \\
\hline 20418 & 64 PSCL & L22 & L465 & $\mathrm{T}$ & $\mathrm{N}$ & 1.877 & 0.533 & 0.829 & 92.0 & 0 & & & & & & \\
\hline 20418 & $65 \mathrm{PSCL}$ & L22 & L722 & V & $\mathrm{P}$ & 1.906 & 0.525 & 0.737 & 88.2 & 0 & 1.925 & 0.756 & 89.9 & 1.925 & 0.756 & 89.9 \\
\hline 20418 & 65PSCL & L22 & L722 & V & $\mathrm{N}$ & 1.944 & 0.514 & 0.775 & 91.5 & 0 & & & & & & \\
\hline 20418 & $65 \mathrm{PSCL}$ & L22 & L722 & $\mathrm{L}$ & $P$ & 1.675 & 0.597 & 0.839 & 90.1 & 2 & 1.817 & 0.863 & 93.9 & 1.979 & 0.807 & 95.1 \\
\hline 20418 & $65 \mathrm{PSCL}$ & L22 & L722 & $\mathrm{L}$ & $\mathrm{N}$ & 1.959 & 0.510 & 0.887 & 97.7 & 1 & & & & & & \\
\hline 20418 & 65PSCL & L22 & L722 & $\mathrm{T}$ & $\mathrm{P}$ & 2.114 & 0.473 & 0.775 & 98.0 & 1 & 2.142 & 0.751 & 96.3 & & & \\
\hline 20418 & $65 \mathrm{PSCL}$ & L22 & L722 & $\mathrm{T}$ & $\mathrm{N}$ & 2.169 & 0.461 & 0.727 & 94.5 & 0 & & & & & & \\
\hline 20418 & 66PSCL & L22 & L861 (961) & V & $\mathrm{P}$ & 1.975 & 0.506 & 0.769 & 92.6 & 1 & 2.010 & 0.790 & 94.2 & 2.010 & 0.790 & 94.2 \\
\hline 20418 & $66 \mathrm{PSCL}$ & L22 & L861 (961) & V & $\mathrm{N}$ & 2.046 & 0.489 & 0.810 & 95.8 & 1 & & & & & & \\
\hline 20418 & 66PSCL & L22 & L861 (961) & $\mathrm{L}$ & $\mathrm{P}$ & 1.931 & 0.518 & 0.761 & 92.5 & 1 & 2.064 & 0.785 & 96.1 & 2.033 & 0.784 & 95.2 \\
\hline 20418 & 66PSCL & L22 & L861 (961) & $\mathrm{L}$ & $\mathrm{N}$ & 2.197 & 0.455 & 0.809 & 99.6 & 1 & & & & & & \\
\hline 20418 & 66 PSCL & L22 & L861 (961) & $\mathrm{T}$ & $\mathrm{P}$ & 2.053 & 0.487 & 0.778 & 94.7 & 1 & 2.002 & 0.782 & 94.3 & & & \\
\hline 20418 & 66PSCL & L22 & L861 (961) & $\mathrm{T}$ & $\mathrm{N}$ & 1.952 & 0.512 & 0.787 & 93.8 & 1 & & & & & & \\
\hline 20418 & 67 PSCL & L22 & L727 & V & $\mathrm{P}$ & 1.954 & 0.512 & 0.734 & 90.6 & 0 & 1.943 & 0.735 & 90.3 & 1.943 & 0.735 & 90.3 \\
\hline 20418 & 67PSCL & L22 & L727 & V & $\mathrm{N}$ & 1.932 & 0.518 & 0.735 & 90.0 & 1 & & & & & & \\
\hline 20418 & 67PSCL & L22 & L727 & $L$ & $\mathrm{P}$ & 1.739 & 0.575 & 0.766 & 86.5 & 1 & 1.746 & 0.785 & 87.9 & 1.910 & 0.800 & 85.6 \\
\hline 20418 & 67PSCL & L22 & L727 & $L$ & $\mathrm{~N}$ & 1.754 & 0.570 & 0.804 & 89.2 & 1 & & & & & & \\
\hline 20418 & 67PSCL & L22 & L727 & $\mathrm{T}$ & $\mathrm{P}$ & 2.057 & 0.486 & 0.858 & 85.5 & 0 & 2.074 & 0.815 & 83.3 & & & \\
\hline 20418 & 67PSCL & L22 & L727 & $\mathrm{T}$ & $\mathrm{N}$ & 2.090 & 0.478 & 0.772 & 81.0 & 0 & & & & & & \\
\hline 20418 & 68PSCL & L22 & L975 & V & $P$ & 1.985 & 0.504 & 0.685 & 83.9 & 0 & 1.985 & 0.687 & 83.7 & 1.985 & 0.687 & 83.7 \\
\hline 20418 & 68PSCL & L22 & L975 & V & $\mathrm{N}$ & 1.985 & 0.504 & 0.688 & 83.5 & 0 & & & & & & \\
\hline 20418 & 68PSCL & L22 & L975 & $\mathrm{L}$ & $\mathrm{P}$ & 2.498 & 0.400 & 0.532 & 79.0 & 0 & 2.517 & 0.544 & 80.5 & 2.247 & 0.634 & 83.4 \\
\hline 20418 & 68PSCL & L22 & L975 & $L$ & $\mathrm{~N}$ & 2.537 & 0.394 & 0.557 & 81.9 & 0 & & & & & & \\
\hline 20418 & 68PSCL & L22 & L975 & $T$ & $\mathrm{P}$ & 1.984 & 0.504 & 0.730 & 86.3 & 0 & 1.977 & 0.723 & 86.3 & & & \\
\hline 20418 & 68PSCL & L22 & L975 & $\mathrm{T}$ & $\mathrm{N}$ & 1.971 & 0.507 & 0.717 & 86.3 & 0 & & & & & & \\
\hline
\end{tabular}




\begin{tabular}{|c|c|c|c|c|c|c|c|c|c|c|c|c|c|c|c|c|}
\hline 20402 & $71 \mathrm{PSCL}$ & L22 & L1506 & V & $P$ & 2.358 & 0.424 & 0.787 & 101.4 & 0 & 2.334 & 0.787 & 101.2 & 2.334 & 0.787 & 101.2 \\
\hline 20402 & $71 \mathrm{PSCL}$ & L22 & L1506 & V & $\mathrm{N}$ & 2.309 & 0.433 & 0.787 & 100.9 & 1 & & & & & & \\
\hline 20402 & $71 \mathrm{PSCL}$ & L22 & L1506 & $\mathrm{L}$ & $\mathrm{P}$ & 2.577 & 0.388 & 0.629 & 92.7 & 0 & 2.558 & 0.634 & 93.6 & 2.248 & 0.718 & 93.4 \\
\hline 20402 & $71 \mathrm{PSCL}$ & L22 & L1506 & $\mathrm{L}$ & $\mathrm{N}$ & 2.538 & 0.394 & 0.639 & 94.5 & 0 & & & & & & \\
\hline 20402 & $71 \mathrm{PSCL}$ & L22 & L1506 & $\mathrm{T}$ & $\mathrm{P}$ & 1.949 & 0.513 & 0.803 & 93.5 & 1 & 1.938 & 0.803 & 93.2 & & & \\
\hline 20402 & $71 \mathrm{PSCL}$ & L22 & L1506 & $\mathrm{T}$ & $\mathrm{N}$ & 1.927 & 0.519 & 0.802 & 92.9 & 1 & & & & & & \\
\hline 20402 & 71 PSCL & L22 & L1506 & V & $\mathrm{P}$ & 2.353 & 0.425 & 0.776 & 100.8 & 1 & 2.331 & 0.768 & 100.3 & 2.331 & 0.768 & 100.3 \\
\hline 20402 & 71 PSCL & L22 & L1506 & V & $\mathrm{N}$ & 2.309 & 0.433 & 0.759 & 99.7 & 1 & & & & & & \\
\hline 20402 & $71 \mathrm{PSCL}$ & L22 & L1506 & $L$ & $\mathrm{P}$ & 2.551 & 0.392 & 0.622 & 91.8 & 0 & 2.522 & 0.625 & 92.5 & 2.248 & 0.722 & 93.8 \\
\hline 20402 & $71 \mathrm{PSCL}$ & L22 & L1506 & $L$ & $\mathrm{~N}$ & 2.494 & 0.401 & 0.628 & 93.1 & 0 & & & & & & \\
\hline 20402 & $71 \mathrm{PSCL}$ & L22 & L1506 & $\mathrm{T}$ & $\mathrm{P}$ & 1.992 & 0.502 & 0.825 & 95.8 & 1 & 1.974 & 0.818 & 95.1 & & & \\
\hline 20402 & $71 \mathrm{PSCL}$ & L22 & L1506 & $\mathrm{T}$ & $\mathrm{N}$ & 1.957 & 0.511 & 0.811 & 94.4 & 1 & & & & & & \\
\hline 20427 & 74 PSCL & L22 & L1490 & $\mathrm{V}$ & $\mathrm{P}$ & 2.013 & 0.497 & 0.748 & 93.2 & 1 & 2.025 & 0.757 & 94.0 & 2.025 & 0.757 & 94.0 \\
\hline 20427 & 74 PSCL & L22 & L1490 & V & $\mathrm{N}$ & 2.037 & 0.491 & 0.766 & 94.7 & 0 & & & & & & \\
\hline 20427 & 74 PSCL & L22 & L1490 & $\mathrm{L}$ & $\mathrm{P}$ & 2.232 & 0.448 & 0.662 & 90.0 & 0 & 2.194 & 0.685 & 91.9 & 2.208 & 0.713 & 92.9 \\
\hline 20427 & 74 PSCL & L22 & L1490 & $\mathrm{L}$ & $\mathrm{N}$ & 2.155 & 0.464 & 0.707 & 93.8 & 0 & & & & & & \\
\hline 20427 & 74 PSCL & L22 & L1490 & $\mathrm{T}$ & $\mathrm{P}$ & 2.150 & 0.465 & 0752 & 94.8 & 0 & 2.223 & 0.742 & 94.0 & & & \\
\hline 20427 & 74 PSCL & L22 & L1490 & $\mathrm{T}$ & $\mathrm{N}$ & 2.295 & 0.436 & 0.732 & 93.1 & 0 & & & & & & \\
\hline 30729 & 74 PSCL & L22 & L1490 & V & $\mathrm{P}$ & 2.078 & 0.481 & 0.794 & 96.9 & 1 & 2.036 & 0.771 & 94.8 & 2.036 & 0.771 & 94.8 \\
\hline 30729 & 74 PSCL & L22 & L1490 & V & $\mathrm{N}$ & 1.994 & 0.501 & 0.748 & 92.7 & 1 & & & & & & \\
\hline 30729 & 74 PSCL & L22 & L1490 & $L$ & $\mathrm{P}$ & 2.273 & 0.440 & 0.696 & 92.6 & 0 & 2.192 & 0.692 & 92.2 & 2.180 & 0.704 & 91.9 \\
\hline 30729 & 74 PSCL & L22 & L1490 & $\mathrm{L}$ & $\mathrm{N}$ & 2.111 & 0.474 & 0.689 & 91.8 & 1 & & & & & & \\
\hline 30729 & 74 PSCL & L22 & L1490 & $\mathrm{T}$ & $\mathrm{P}$ & 2.099 & 0.477 & 0.734 & 93.0 & 0 & 2.169 & 0.715 & 91.7 & & & \\
\hline 30729 & 74 PSCL & L22 & L1490 & $\mathrm{T}$ & $\mathrm{N}$ & 2.239 & 0.447 & 0.696 & 90.4 & 0 & & & & & & \\
\hline 20418 & 75PSCL & L22 & L498 & V & $\mathrm{P}$ & 1.143 & 0.875 & 0.031 & $11.1 \mathrm{bad}$ & & 1.574 & 0.399 & 50.6 & & & \\
\hline 20418 & 75 PSCL & L22 & L498 & V & $\mathrm{N}$ & 2.004 & 0.499 & 0.767 & $90.0 \mathrm{bad}$ & & & & & & & \\
\hline 20418 & 75PSCL & L22 & L498 & $L$ & $\mathrm{P}$ & 1.965 & 0.509 & 0.754 & 88.4 & 0 & 1.910 & 0.735 & 86.7 & 1.841 & 0.780 & 87.7 \\
\hline 20418 & 75PSCL & L22 & L498 & $\mathrm{L}$ & $\mathrm{N}$ & 1.855 & 0.539 & 0.715 & 85.0 & 0 & & & & & & \\
\hline 20418 & $75 \mathrm{PSCL}$ & L22 & L498 & $\mathrm{T}$ & $\mathrm{P}$ & 1.695 & 0.590 & 0.805 & 86.9 & 1 & 1.772 & 0.826 & 88.7 & & & \\
\hline 20418 & $75 \mathrm{PSCL}$ & L22 & L498 & $\mathrm{T}$ & $\mathrm{N}$ & 1.848 & 0.541 & 0.846 & 90.5 & 0 & & & & & & \\
\hline
\end{tabular}




\begin{tabular}{|c|c|c|c|c|c|c|c|c|c|c|c|c|c|c|c|c|}
\hline 30729 & $75 \mathrm{PSCL}$ & L22 & L498 & V & $P$ & 10.753 & 0.093 & 0.283 & $83.2 \mathrm{bad}$ & & 5.948 & 0.157 & 47.1 & & & \\
\hline 30729 & $75 \mathrm{PSCL}$ & L22 & L498 & V & $\mathrm{N}$ & 1.143 & 0.875 & 0.031 & $11.1 \mathrm{bad}$ & & & & & & & \\
\hline 30729 & 75PSCL & L22 & L498 & $\mathrm{L}$ & $P$ & 2.004 & 0.499 & 0.767 & 90.0 & 0 & 1.961 & 0.763 & 89.3 & 1.850 & 0.782 & 88.1 \\
\hline 30729 & 75PSCL & L22 & L498 & $\mathrm{L}$ & $\mathrm{N}$ & 1.918 & 0.521 & 0.758 & 88.6 & 0 & & & & & & \\
\hline 30729 & $75 \mathrm{PSCL}$ & L22 & L498 & $\mathrm{T}$ & $\mathrm{P}$ & 1.672 & 0.598 & 0.804 & 86.1 & 1 & 1.739 & 0.802 & 86.8 & & & \\
\hline 30729 & 75PSCL & L22 & L498 & $\mathrm{T}$ & $\mathrm{N}$ & 1.807 & 0.553 & 0.800 & 87.6 & 0 & & & & & & \\
\hline 20418 & 76 PSCL & L22 & L495 & V & $\mathrm{P}$ & 2.042 & 0.490 & 0.699 & 90.3 & 0 & 2.015 & 0.709 & 90.7 & 2.015 & 0.709 & 90.7 \\
\hline 20418 & 76 PSCL & L22 & L495 & V & $\mathrm{N}$ & 1.987 & 0.503 & 0.720 & 91.1 & 1 & & & & & & \\
\hline 20418 & 76 PSCL & L22 & L495 & $\mathrm{L}$ & $\mathrm{P}$ & 2.074 & 0.482 & 0.756 & 92.2 & 0 & 2.002 & 0.749 & 91.5 & 2.072 & 0.758 & 94.2 \\
\hline 20418 & 76 PSCL & L22 & L495 & $\mathrm{L}$ & $\mathrm{N}$ & 1.929 & 0.518 & 0.743 & 90.8 & 1 & & & & & & \\
\hline 20418 & 76 PSCL & L22 & L495 & $\mathrm{T}$ & $\mathrm{P}$ & 2.276 & 0.439 & 0.796 & 100.4 & 1 & 2.142 & 0.766 & 96.9 & & & \\
\hline 20418 & 76 PSCL & L22 & L495 & $\mathrm{T}$ & $\mathrm{N}$ & 2.008 & 0.498 & 0.737 & 93.3 & 2 & & & & & & \\
\hline 20227 & 77PSCL & L22 & L497 & V & $\mathrm{P}$ & 2.097 & 0.477 & 0.791 & 93.5 & 0 & 2.079 & 0.789 & 93.5 & 2.079 & 0.789 & 93.5 \\
\hline 20227 & 77PSCL & L22 & L497 & V & $\mathrm{N}$ & 2.060 & 0.485 & 0.787 & 93.6 & 0 & & & & & & \\
\hline 20227 & 77PSCL & L22 & L497 & $\mathrm{L}$ & $\mathrm{P}$ & 2.126 & 0.470 & 0.768 & 89.2 & 0 & 2.108 & 0.801 & 91.2 & 2.079 & 0.803 & 92.2 \\
\hline 20227 & 77PSCL & L22 & L497 & $L$ & $\mathrm{~N}$ & 2.090 & 0.479 & 0.833 & 93.2 & 0 & & & & & & \\
\hline 20227 & 77PSCL & L22 & L497 & $\mathrm{T}$ & $\mathrm{P}$ & 2.109 & 0.474 & 0.797 & 92.9 & 0 & 2.050 & 0.806 & 93.3 & & & \\
\hline 20227 & 77PSCL & L22 & L497 & $\mathrm{T}$ & $\mathrm{N}$ & 1.990 & 0.502 & 0.816 & 93.7 & 0 & & & & & & \\
\hline 20227 & 79 PSCL & L22 & L451 & V & $\mathrm{P}$ & 2.139 & 0.467 & 0.672 & 86.4 & 0 & 2.129 & 0.685 & 87.6 & 2.129 & 0.685 & 87.6 \\
\hline 20227 & 79 PSCL & L22 & L451 & V & $\mathrm{N}$ & 2.119 & 0.472 & 0.698 & 88.7 & 0 & & & & & & \\
\hline 20227 & 79 PSCL & L22 & L451 & $\mathrm{L}$ & $\mathrm{P}$ & 2.332 & 0.429 & 0.620 & 86.4 & 0 & 2.325 & 0.643 & 88.7 & 2.171 & 0.693 & 87.8 \\
\hline 20227 & 79PSCL & L22 & L451 & $\mathrm{L}$ & $\mathrm{N}$ & 2.317 & 0.432 & 0.666 & 91.1 & 0 & & & & & & \\
\hline 20227 & 79 PSCL & L22 & L451 & $\mathrm{T}$ & $\mathrm{P}$ & 2.107 & 0.475 & 0.745 & 87.6 & 0 & 2.018 & 0.743 & 86.9 & & & \\
\hline 20227 & 79PSCL & L22 & L451 & $\mathrm{T}$ & $\mathrm{N}$ & 1.929 & 0.518 & 0.741 & 86.3 & 0 & & & & & & \\
\hline 20227 & 80 PSCL & L22 & L1499 & V & $\mathrm{P}$ & 2.017 & 0.496 & 0.750 & 93.3 & 1 & 2.056 & 0.762 & 94.7 & 2.056 & 0.762 & 94.7 \\
\hline 20227 & 80 PSCL & L22 & L1499 & $\mathrm{V}$ & $\mathrm{N}$ & 2.094 & 0.478 & 0.774 & 96.2 & 1 & & & & & & \\
\hline 20227 & 80 PSCL & L22 & L1499 & $\mathrm{L}$ & $\mathrm{P}$ & 2.307 & 0.434 & 0.697 & 94.6 & 0 & 2.258 & 0.714 & 95.4 & 2.217 & 0.759 & 97.2 \\
\hline 20227 & 80 PSCL & L22 & L1499 & $\mathrm{L}$ & $\mathrm{N}$ & 2.209 & 0.453 & 0.732 & 96.3 & 1 & & & & & & \\
\hline 20227 & 80 PSCL & L22 & L1499 & $\mathrm{T}$ & $P$ & 2.181 & 0.459 & 0.820 & 100.0 & 1 & 2.176 & 0.804 & 99.0 & & & \\
\hline 20227 & $80 \mathrm{PSCL}$ & L22 & L1499 & $\mathrm{T}$ & $\mathrm{N}$ & 2.170 & 0.461 & 0.788 & 98.0 & 1 & & & & & & \\
\hline
\end{tabular}




\begin{tabular}{|c|c|c|c|c|c|c|c|c|c|c|c|c|c|c|c|c|}
\hline 20227 & $81 \mathrm{PSCL}$ & L22 & L958 & V & $\mathrm{P}$ & 2.072 & 0.483 & 0.781 & 93.0 & 0 & 2.084 & 0.792 & 94.2 & 2.084 & 0.792 & 94 \\
\hline 20227 & $81 \mathrm{PSCL}$ & L22 & L958 & V & $\mathrm{N}$ & 2.097 & 0.477 & 0.803 & 95.3 & 0 & & & & & & \\
\hline 20227 & $81 \mathrm{PSCL}$ & L22 & L958 & $\mathrm{L}$ & $\mathrm{P}$ & 1.850 & 0.541 & 0.760 & 87.5 & 0 & 1.893 & 0.761 & 87.8 & 1.905 & 0.780 & 89. \\
\hline 20227 & $81 \mathrm{PSCL}$ & L22 & L958 & $\mathrm{L}$ & $\mathrm{N}$ & 1.935 & 0.517 & 0.763 & 88.0 & 0 & & & & & & \\
\hline 20227 & $81 \mathrm{PSCL}$ & L22 & L958 & $\mathrm{T}$ & $P$ & 1.952 & 0.512 & 0.812 & 90.7 & 0 & 1.917 & 0.798 & 90.2 & & & \\
\hline 20227 & 81 PSCL & L22 & L958 & $\mathrm{T}$ & $\mathrm{N}$ & 1.883 & 0.531 & 0.785 & 89.7 & 0 & & & & & & \\
\hline 20418 & 82 PSCL & L22 & L473 & V & $P$ & 1.918 & 0.521 & 0.734 & 87.0 & 0 & 1.890 & 0.725 & 85.9 & 1.890 & 0.725 & 85. \\
\hline 20418 & $82 \mathrm{PSCL}$ & L22 & L473 & V & $\mathrm{N}$ & 1.862 & 0.537 & 0.716 & 84.8 & 0 & & & & & & \\
\hline 20418 & $82 \mathrm{PSCL}$ & L22 & L473 & $\mathrm{L}$ & $P$ & 2.252 & 0.444 & 0.660 & 93.0 & 0 & 2.288 & 0.651 & 91.3 & 2.098 & 0.730 & 91. \\
\hline 20418 & 82 PSCL & L22 & L473 & $\mathrm{L}$ & $\mathrm{N}$ & 2.323 & 0.430 & 0.642 & 89.6 & 0 & & & & & & \\
\hline 20418 & 82 PSCL & L22 & L473 & $\mathrm{T}$ & $\mathrm{P}$ & 1.989 & 0.503 & 0.811 & 91.5 & 0 & 1.908 & 0.808 & 90.9 & & & \\
\hline 20418 & $82 \mathrm{PSCL}$ & L22 & L473 & $\mathrm{T}$ & $\mathrm{N}$ & 1.827 & 0.547 & 0.804 & 90.4 & 1 & & & & & & \\
\hline 20418 & 83 PSCL & L22 & L496 & V & $P$ & 2.015 & 0.496 & 0.798 & 90.5 & 0 & 2.053 & 0.803 & 90.5 & 2.053 & 0.803 & 90 \\
\hline 20418 & $83 \mathrm{PSCL}$ & L22 & L496 & V & $\mathrm{N}$ & 2.091 & 0.478 & 0.808 & 90.6 & 0 & & & & & & \\
\hline 20418 & 83 PSCL & L22 & L496 & $\mathrm{L}$ & $\mathrm{P}$ & 2.300 & 0.435 & 0.708 & 90.2 & 0 & 2.353 & 0.704 & 89.9 & 2.258 & 0.687 & 89. \\
\hline 20418 & 83 PSCL & L22 & L496 & $\mathrm{L}$ & $\mathrm{N}$ & 2.406 & 0.416 & 0.701 & 89.7 & 0 & & & & & & \\
\hline 20418 & 83 PSCL & L22 & L496 & $\mathrm{T}$ & $P$ & 2.224 & 0.450 & 0.675 & 89.1 & 0 & 2.163 & 0.669 & 88.4 & & & \\
\hline 20418 & 83 PSCL & L22 & L496 & $\mathrm{T}$ & $\mathrm{N}$ & 2.102 & 0.476 & 0.663 & 87.7 & 0 & & & & & & \\
\hline 20402 & 84 PSCL & L22 & L781 & V & $P$ & 2.215 & 0.452 & 0.616 & 82.3 & 0 & 2.244 & 0.659 & 84.7 & 2.244 & 0.659 & 84. \\
\hline 20402 & 84 PSCL & L22 & L781 & V & $\mathrm{N}$ & 2.273 & 0.440 & 0.701 & 87.1 & 0 & & & & & & \\
\hline 20402 & 84 PSCL & L22 & L781 & $\mathrm{L}$ & $\mathrm{P}$ & 2.287 & 0.437 & 0.745 & 96.8 & 0 & 2.243 & 0.765 & 97.7 & 2.168 & 0.749 & 92. \\
\hline 20402 & 84 PSCL & L22 & L781 & $\mathrm{L}$ & $\mathrm{N}$ & 2.198 & 0.455 & 0.785 & 98.7 & 0 & & & & & & \\
\hline 20402 & 84 PSCL & L22 & L781 & $\mathrm{T}$ & $\mathrm{P}$ & 2.149 & 0.465 & 0.725 & 86.8 & 0 & 2.094 & 0.733 & 87.9 & & & \\
\hline 20402 & 84 PSCL & L22 & L781 & $\mathrm{T}$ & $\mathrm{N}$ & 2.039 & 0.490 & 0.742 & 89.1 & 0 & & & & & & \\
\hline 20402 & $85 \mathrm{PSCL}$ & L22 & L464 & V & $P$ & 2.264 & 0.442 & 0.699 & 90.3 & 0 & 2.217 & 0.681 & 89.0 & 2.217 & 0.681 & 09. \\
\hline 20402 & $85 \mathrm{PSCL}$ & L22 & L464 & V & $\mathrm{N}$ & 2.169 & 0.461 & 0.663 & 87.7 & 0 & & & & & & \\
\hline 20402 & $85 \mathrm{PSCL}$ & L22 & L464 & $\mathrm{L}$ & $\mathrm{P}$ & 2.947 & 0.339 & 0.572 & 90.3 & 0 & 2.923 & 0.570 & 90.8 & 2.823 & 0.554 & 83. \\
\hline 20402 & $85 \mathrm{PSCL}$ & L22 & L464 & $\mathrm{L}$ & $\mathrm{N}$ & 2.899 & 0.345 & 0.568 & 91.3 & 0 & & & & & & \\
\hline 20402 & $85 \mathrm{PSCL}$ & L22 & L464 & $\mathrm{T}$ & $\mathrm{P}$ & 2.727 & 0.367 & 0.540 & 67.1 & 0 & 2.723 & 0.538 & 76.3 & & & \\
\hline 20402 & $85 \mathrm{PSCL}$ & L22 & L464 & $\mathrm{T}$ & $\mathrm{N}$ & 2.718 & 0.368 & 0.537 & 85.5 & 0 & & & & & & \\
\hline
\end{tabular}




\begin{tabular}{|c|c|c|c|c|c|c|c|c|c|c|c|c|c|c|c|c|}
\hline 20222 & $96 \mathrm{PSCL}$ & L22 & L450 & V & $P$ & 2.090 & 0.478 & 0.728 & 90.0 & 0 & 2.082 & 0.732 & 90.9 & 2.082 & 0.732 & 90.9 \\
\hline 20222 & $96 \mathrm{PSCL}$ & L22 & L450 & $\mathrm{V}$ & $\mathrm{N}$ & 2.074 & 0.482 & 0.736 & 91.7 & 0 & & & & & & \\
\hline 20222 & $96 \mathrm{PSCL}$ & L22 & L450 & $\mathrm{L}$ & $\mathrm{P}$ & 2.606 & 0.384 & 0.490 & 82.2 & 0 & 2.523 & 0.496 & 81.8 & 2.313 & 0.572 & 84.4 \\
\hline 20222 & $96 \mathrm{PSCL}$ & L22 & L450 & $\mathrm{L}$ & $\mathrm{N}$ & 2.439 & 0.410 & 0.502 & 81.3 & 0 & & & & & & \\
\hline 20222 & $96 \mathrm{PSCL}$ & L22 & L450 & $\mathrm{T}$ & $\mathrm{P}$ & 4.252 & 0.235 & 0.273 & 4.8bad & & 2.104 & 0.649 & 86.9 & & & \\
\hline 20222 & 96PSCL & L22 & L450 & $\mathrm{T}$ & $\mathrm{N}$ & 2.104 & 0.475 & 0.649 & 86.9 & 0 & & & & & & \\
\hline 20427 & $96 \mathrm{PSCL}$ & L22 & L450 & $\mathrm{V}$ & $\mathrm{P}$ & 2.075 & 0.482 & 0.721 & 89.1 & 0 & 2.066 & 0.725 & 89.9 & 2.066 & 0.725 & 89.9 \\
\hline 20427 & $96 \mathrm{PSCL}$ & L22 & L450 & $\mathrm{V}$ & $\mathrm{N}$ & 2.058 & 0.486 & 0.728 & 90.7 & 0 & & & & & & \\
\hline 20427 & $96 \mathrm{PSCL}$ & L22 & L450 & $\mathrm{L}$ & $\mathrm{P}$ & 2.568 & 0.389 & 0.470 & 78.1 & 0 & 2.548 & 0.474 & 78.9 & 2.370 & 0.564 & 83.0 \\
\hline 20427 & $96 \mathrm{PSCL}$ & L22 & L450 & $\mathrm{L}$ & $\mathrm{N}$ & 2.528 & 0.396 & 0.478 & 79.6 & 0 & & & & & & \\
\hline 20427 & $96 \mathrm{PSCL}$ & L22 & L450 & $\mathrm{T}$ & $P$ & 2.244 & 0.446 & 0.641 & 86.3 & 0 & 2.191 & 0.654 & 87.1 & & & \\
\hline 20427 & $96 \mathrm{PSCL}$ & L22 & L450 & $\mathrm{T}$ & $\mathrm{N}$ & 2.139 & 0.468 & 0.667 & 87.9 & 0 & & & & & & \\
\hline 20222 & 98 USGS & L22 & L529 & $\mathrm{V}$ & $\mathrm{P}$ & 1.929 & 0.518 & 0.802 & 51.5 & 0 & 1.931 & 0.816 & 52.0 & 1.931 & 0.816 & 52.0 \\
\hline 20222 & 98 USGS & L22 & L529 & V & $\mathrm{N}$ & 1.933 & 0.517 & 0.829 & 52.6 & 0 & & & & & & \\
\hline 20222 & 98 USGS & L22 & L529 & $\mathrm{L}$ & $\mathrm{P}$ & 2.006 & 0.498 & 0.833 & 52.3 & 0 & 2.002 & 0.829 & 52.0 & 2.129 & 0.844 & 55.3 \\
\hline 20222 & 98USGS & L22 & L529 & $\mathrm{L}$ & $\mathrm{N}$ & 1.998 & 0.501 & 0.824 & 51.7 & 0 & & & & & & \\
\hline 20222 & 98 USGS & L22 & L529 & $\mathrm{T}$ & $\mathrm{P}$ & 2.280 & 0.439 & 0.871 & 58.6 & 0 & 2.256 & 0.860 & 58.5 & & & \\
\hline 20222 & 98 USGS & L22 & L529 & $\mathrm{T}$ & $\mathrm{N}$ & 2.232 & 0.448 & 0.849 & 58.5 & 0 & & & & & & \\
\hline 20314 & $111 \mathrm{PSCL}$ & L22 & L959 & V & $\mathrm{P}$ & 2.073 & 0.482 & 0.728 & 90.1 & 0 & 2.062 & 0.727 & 89.4 & 2.062 & 0.727 & 89.4 \\
\hline 20314 & $111 \mathrm{PSCL}$ & L22 & L959 & $\mathrm{V}$ & $\mathrm{N}$ & 2.051 & 0.488 & 0.726 & 88.7 & 0 & & & & & & \\
\hline 20314 & $111 \mathrm{PSCL}$ & L22 & L959 & $\mathrm{L}$ & $\mathrm{P}$ & 2.498 & 0.400 & 0.574 & 84.6 & 0 & 2.476 & 0.576 & 85.2 & 2.517 & 0.566 & 85.3 \\
\hline 20314 & 111 PSCL & L22 & L959 & $\mathrm{L}$ & $\mathrm{N}$ & 2.454 & 0.408 & 0.578 & 85.8 & 0 & & & & & & \\
\hline 20314 & $111 \mathrm{PSCL}$ & L22 & L959 & $\mathrm{T}$ & $\mathrm{P}$ & 2.616 & 0.382 & 0.560 & 85.9 & 0 & 2.559 & 0.556 & 85.4 & & & \\
\hline 20314 & $111 \mathrm{PSCL}$ & L22 & L959 & $\mathrm{T}$ & $\mathrm{N}$ & 2.501 & 0.400 & 0.553 & 84.9 & 0 & & & & & & \\
\hline 20314 & 113PSCL & L22 & L962 & V & $\mathrm{P}$ & 15.723 & 0.064 & 0.627 & $224.9 \mathrm{bad}$ & & 1.978 & 0.704 & 85.4 & 1.978 & 0.704 & 85.4 \\
\hline 20314 & 113PSCL & L22 & L962 & $\mathrm{V}$ & $\mathrm{N}$ & 1.978 & 0.506 & 0.704 & 85.4 noisy & & & & & & & \\
\hline 20314 & 113PSCL & L22 & L962 & $\mathrm{L}$ & $\mathrm{P}$ & 2.200 & 0.455 & 0.718 & 85.6noisy & & 2.157 & 0.736 & 86.9 & 2.179 & 0.714 & 87.6 \\
\hline 20314 & 113PSCL & L22 & L962 & $\mathrm{L}$ & $\mathrm{N}$ & 2.113 & 0.473 & 0.753 & 88.3noisy & & & & & & & \\
\hline 20314 & 113PSCL & L22 & L962 & $\mathrm{T}$ & $\mathrm{P}$ & 2.202 & 0.454 & 0.693 & 88.3noisy & & 2.202 & 0.693 & 88.3 & & & \\
\hline 20314 & 113PSCL & L22 & L962 & $\mathrm{T}$ & $\mathrm{N}$ & & & & & & & & & & & \\
\hline
\end{tabular}




\begin{tabular}{|c|c|c|c|c|c|c|c|c|c|}
\hline 20402 & 118PSCL & L22 & L504 & $\mathrm{V}$ & $P$ & 2.176 & 0.460 & 0.772 & 93.9 \\
\hline 20402 & 118PSCL & L22 & L504 & V & $\mathrm{N}$ & 2.159 & 0.463 & 0.761 & 93.5 \\
\hline 20402 & 118PSCL & L22 & L504 & $\mathrm{L}$ & $P$ & 2.363 & 0.423 & 0.698 & 92.2 \\
\hline 20402 & 118PSCL & L22 & L504 & $\mathrm{L}$ & $\mathrm{N}$ & 2.241 & 0.446 & 0.743 & 94.6 \\
\hline 20402 & 118PSCL & L22 & L504 & $\mathrm{T}$ & $P$ & 1.822 & 0.549 & 0.846 & 89.4 \\
\hline 20402 & 118PSCL & L22 & L504 & $\mathrm{T}$ & $\mathrm{N}$ & 1.784 & 0.560 & 0.838 & 86.9 \\
\hline 20402 & $143 \mathrm{PSCL}$ & L22 & L1502 & V & $P$ & 2.058 & 0.486 & 0.775 & 94.9 \\
\hline 20402 & $143 \mathrm{PSCL}$ & L22 & L1502 & V & $\mathrm{N}$ & 2.058 & 0.486 & 0.827 & 97.6 \\
\hline 20402 & $143 \mathrm{PSCL}$ & L22 & L1502 & $\mathrm{L}$ & $P$ & 1.859 & 0.538 & 0.817 & 91.3 \\
\hline 20402 & 143 PSCL & L22 & L1502 & $\mathrm{L}$ & $\mathrm{N}$ & 1.742 & 0.574 & 0.818 & 89.1 \\
\hline 20402 & $143 \mathrm{PSCL}$ & L22 & L1502 & $\mathrm{T}$ & $\mathrm{P}$ & 1.745 & 0.573 & 0.855 & 91.0 \\
\hline 20402 & $143 \mathrm{PSCL}$ & L22 & L1502 & $\mathrm{T}$ & $\mathrm{N}$ & 1.704 & 0.587 & 0.874 & 91.3 \\
\hline 30207 & 143 PSCL & L22 & L1502 & V & $\mathrm{P}$ & 2.131 & 0.469 & 0.831 & 99.4 \\
\hline 30207 & $143 \mathrm{PSCL}$ & L22 & L1502 & V & $\mathrm{N}$ & 2.003 & 0.499 & 0.775 & 94.0 \\
\hline 30207 & $143 \mathrm{PSCL}$ & L22 & L1502 & $\mathrm{L}$ & $\mathrm{P}$ & 1.893 & 0.528 & 0.834 & 93.2 \\
\hline 30207 & $143 \mathrm{PSCL}$ & L22 & L1502 & $\mathrm{L}$ & $\mathrm{N}$ & 1.756 & 0.570 & 0.817 & 89.7 \\
\hline 30207 & $143 \mathrm{PSCL}$ & L22 & L1502 & $\mathrm{T}$ & $\mathrm{P}$ & 1.777 & 0.563 & 0.869 & 92.8 \\
\hline 30207 & $143 \mathrm{PSCL}$ & L22 & L1502 & $\mathrm{T}$ & $\mathrm{N}$ & 1.655 & 0.604 & 0.854 & 89.1 \\
\hline 20402 & 144 PSCL & L22 & L966 & V & $\mathrm{P}$ & 2.188 & 0.457 & 0.751 & 94.1 \\
\hline 20402 & 144 PSCL & L22 & L966 & $\mathrm{V}$ & $\mathrm{N}$ & 2.132 & 0.469 & 0.735 & 92.0 \\
\hline 20402 & $144 \mathrm{PSCL}$ & L22 & L966 & $\mathrm{L}$ & $\mathrm{P}$ & 2.092 & 0.478 & 0.689 & 87.3 \\
\hline 20402 & $144 \mathrm{PSCL}$ & L22 & L966 & $\mathrm{L}$ & $\mathrm{N}$ & 2.012 & 0.497 & 0.662 & 84.9 \\
\hline 20402 & $144 \mathrm{PSCL}$ & L22 & L966 & $\mathrm{T}$ & $P$ & 2.174 & 0.460 & 0.803 & 97.0 \\
\hline 20402 & 144 PSCL & L22 & L966 & $\mathrm{T}$ & $\mathrm{N}$ & 2.105 & 0.475 & 0.748 & 92.6 \\
\hline 20402 & $144 \mathrm{PSCL}$ & L22 & L966 & V & $\mathrm{P}$ & 2.227 & 0.449 & 0.777 & 96.4 \\
\hline 20402 & $144 \mathrm{PSCL}$ & L22 & L966 & V & $\mathrm{N}$ & 2.208 & 0.453 & 0.777 & 95.9 \\
\hline 20402 & $144 \mathrm{PSCL}$ & L22 & L966 & $\mathrm{L}$ & $\mathrm{P}$ & 2.088 & 0.479 & 0.679 & 86.8 \\
\hline 20402 & $144 \mathrm{PSCL}$ & L22 & L966 & $\mathrm{L}$ & $\mathrm{N}$ & 2.101 & 0.476 & 0.714 & 89.5 \\
\hline 20402 & $144 \mathrm{PSCL}$ & L22 & L966 & $\mathrm{T}$ & $\mathrm{P}$ & 2.092 & 0.478 & 0.748 & 92.6 \\
\hline 20402 & $144 \mathrm{PSCL}$ & L22 & L966 & $\mathrm{T}$ & $N$ & 2.105 & 0.475 & 0.747 & 93.1 \\
\hline
\end{tabular}

\begin{tabular}{|c|c|c|c|c|c|c|}
\hline 0 & 2.168 & 0.767 & 93.7 & 2.168 & 0.767 & 93.7 \\
\hline 0 & 2.302 & 0.721 & 93.4 & 2.052 & 0.781 & 90.9 \\
\hline 0 & 1.803 & 0.842 & 88.2 & & & \\
\hline 1 & 2.058 & 0.801 & 96.3 & 2.058 & 0.801 & 96.3 \\
\hline 1 & 1.800 & 0.818 & 90.2 & 1.762 & 0.841 & 90.7 \\
\hline 2 & 1.724 & 0.865 & 91.2 & & & \\
\hline 1 & 2.067 & 0.803 & 96.7 & 2.067 & 0.803 & 96.7 \\
\hline 1 & 1.824 & 0.825 & 91.4 & 1.770 & 0.844 & 91.2 \\
\hline 2 & 1.716 & 0.862 & 90.9 & & & \\
\hline 0 & 2.160 & 0.743 & 93.1 & 2.160 & 0.743 & 93.1 \\
\hline 0 & 2.052 & 0.676 & 86.1 & 2.096 & 0.726 & 90.5 \\
\hline 0 & 2.140 & 0.776 & 94.8 & & & \\
\hline 0 & 2.217 & 0.777 & 96.2 & 2.217 & 0.777 & 96.2 \\
\hline 0 & 2.094 & 0.697 & 88.2 & 2.096 & 0.722 & 90.5 \\
\hline 0 & 2.099 & 0.748 & 92.9 & & & \\
\hline
\end{tabular}




\begin{tabular}{|c|c|c|c|c|c|c|c|c|c|c|c|c|c|c|c|c|}
\hline 20402 & 148PSCL & L22 & L485 & V & $\mathrm{P}$ & 2.167 & 0.461 & 0.685 & 89.4 & 0 & 2.188 & 0.700 & 91.3 & 2.188 & 0.700 & 91.3 \\
\hline 20402 & 148 PSCL & L22 & L485 & V & $\mathrm{N}$ & 2.209 & 0.453 & 0.715 & 93.2 & 0 & & & & & & \\
\hline 20402 & 148PSCL & L22 & L485 & $\mathrm{L}$ & $\mathrm{P}$ & 2.283 & 0.438 & 0.661 & 87.3 & 0 & 2.173 & 0.668 & 87.5 & 2.173 & 0.668 & 87.5 \\
\hline 20402 & 148PSCL & L22 & L485 & $\mathrm{L}$ & $\mathrm{N}$ & 2.062 & 0.485 & 0.675 & 87.7 & 0 & & & & & & \\
\hline 20402 & 148PSCL & L22 & L485 & $\mathrm{T}$ & $\mathrm{P}$ & 37.736 & 0.027 & 0.028 & 60.8 dead & & 38.248 & 0.045 & 67.0 & & & \\
\hline 20402 & 148PSCL & L22 & L485 & $\mathrm{T}$ & $\mathrm{N}$ & 38.760 & 0.026 & 0.063 & 73.2dead & & & & & & & \\
\hline 20402 & 169PSCL & L22 & L461 & V & $\mathrm{P}$ & 2.294 & 0.436 & 0.709 & 92.2 & 0 & 2.283 & 0.717 & 93.0 & 2.283 & 0.717 & 93.0 \\
\hline 20402 & 169 PSCL & L22 & L461 & V & $\mathrm{N}$ & 2.273 & 0.440 & 0.725 & 93.8 & 0 & & & & & & \\
\hline 20402 & 169 PSCL & L22 & L461 & $\mathrm{L}$ & $\mathrm{P}$ & 2.794 & 0.358 & 0.518 & 82.5 & 0 & 2.748 & 0.517 & 82.7 & 2.588 & 0.539 & 82.8 \\
\hline 20402 & $169 \mathrm{PSCL}$ & L22 & L461 & $\mathrm{L}$ & $\mathrm{N}$ & 2.703 & 0.370 & 0.516 & 82.9 & 0 & & & & & & \\
\hline 20402 & 169 PSCL & L22 & L461 & $\mathrm{T}$ & $\mathrm{P}$ & 2.465 & 0.406 & 0.558 & 82.1 & 0 & 2.427 & 0.561 & 82.9 & & & \\
\hline 20402 & 169PSCL & L22 & L461 & $\mathrm{T}$ & $\mathrm{N}$ & 2.389 & 0.419 & 0.563 & 83.8 & 0 & & & & & & \\
\hline 20418 & $181 \mathrm{PSCL}$ & L22 & L1496 & V & $\mathrm{P}$ & 1.945 & 0.514 & 0.760 & 91.5 & 1 & 1.921 & 0.747 & 90.3 & 1.921 & 0.747 & 90.3 \\
\hline 20418 & $181 \mathrm{PSCL}$ & L22 & L1496 & V & $\mathrm{N}$ & 1.897 & 0.527 & 0.735 & 89.2 & 1 & & & & & & \\
\hline 20418 & 181 PSCL & L22 & L1496 & $\mathrm{L}$ & $\mathrm{P}$ & 1.917 & 0.522 & 0.828 & 92.9 & 0 & 1.826 & 0.829 & 91.4 & 1.895 & 0.766 & 89.8 \\
\hline 20418 & 181 PSCL & L22 & L1496 & $\mathrm{L}$ & $\mathrm{N}$ & 1.735 & 0.576 & 0.830 & 90.0 & 1 & & & & & & \\
\hline 20418 & $181 \mathrm{PSCL}$ & L22 & L1496 & $\mathrm{T}$ & $\mathrm{P}$ & 2.035 & 0.492 & 0.701 & 88.5 & 0 & 1.964 & 0.703 & 88.2 & & & \\
\hline 20418 & 181 PSCL & L22 & L1496 & $\mathrm{T}$ & $\mathrm{N}$ & 1.893 & 0.528 & 0.705 & 88.0 & 1 & & & & & & \\
\hline 20418 & 182 PSCL & L22 & L457 & V & $\mathrm{P}$ & 2.141 & 0.467 & 0.884 & 93.6 & 0 & 2.126 & 0.859 & 92.2 & 2.126 & 0.859 & 92.2 \\
\hline 20418 & 182 PSCL & L22 & L457 & V & $\mathrm{N}$ & 2.111 & 0.474 & 0.835 & 90.8 & 0 & & & & & & \\
\hline 20418 & 182 PSCL & L22 & L457 & L & $\mathrm{P}$ & 1.894 & 0.528 & 0.693 & 85.7 & 0 & 1.906 & 0.705 & 86.5 & 1.963 & 0.694 & 87.4 \\
\hline 20418 & 182 PSCL & L22 & L457 & $\mathrm{L}$ & $\mathrm{N}$ & 1.919 & 0.521 & 0.717 & 87.4 & 0 & & & & & & \\
\hline 20418 & 182 PSCL & L22 & L457 & $\mathrm{T}$ & $\mathrm{P}$ & 2.016 & 0.496 & 0.680 & 87.8 & 0 & 2.019 & 0.683 & 88.3 & & & \\
\hline 20418 & 182 PSCL & L22 & L457 & $\mathrm{T}$ & $\mathrm{N}$ & 2.021 & 0.495 & 0.686 & 88.8 & 0 & & & & & & \\
\hline 20402 & 183 PSCL & L22 & L479 & V & $\mathrm{P}$ & 2.117 & 0.472 & 0.762 & 89.3 & 0 & 2.115 & 0.751 & 89.6 & 2.115 & 0.751 & 89.9 \\
\hline 20402 & 183 PSCL & L22 & L479 & V & $\mathrm{N}$ & 2.112 & 0.474 & 0.741 & 89.9 & 0 & & & & & & \\
\hline 20402 & 183 PSCL & L22 & L479 & $\mathrm{L}$ & $\mathrm{P}$ & 1.985 & 0.504 & 0.774 & 88.3 & 0 & 1.961 & 0.755 & 87.5 & 1.961 & 0.755 & 87.5 \\
\hline 20402 & 183 PSCL & L22 & L479 & $\mathrm{L}$ & $\mathrm{N}$ & 1.936 & 0.517 & 0.735 & 86.7 & 0 & & & & & & \\
\hline 20402 & 183 PSCL & L22 & L479 & $\mathrm{T}$ & $\mathrm{P}$ & 17.513 & 0.057 & 0.296 & 67.9 dead & & 16.594 & 0.280 & 61.9 & & & \\
\hline 20402 & 183 PSCL & L22 & L479 & $\mathrm{T}$ & $\mathrm{N}$ & 15.674 & 0.064 & 0.264 & 55.9 dead & & & & & & & \\
\hline
\end{tabular}




\begin{tabular}{|c|c|c|c|c|c|c|c|c|c|c|c|c|c|c|c|c|}
\hline 20402 & $186 \mathrm{PSCL}$ & L22 & L483 & V & $\mathrm{P}$ & 1.845 & 0.542 & 0.647 & 84.8 & 1 & 1.837 & 0.648 & 84.8 & 1.837 & 0.648 & 84.8 \\
\hline 20402 & $186 \mathrm{PSCL}$ & L22 & L483 & V & $\mathrm{N}$ & 1.828 & 0.547 & 0.648 & 84.8 & 1 & & & & & & \\
\hline 20402 & $186 \mathrm{PSCL}$ & L22 & L483 & $\mathrm{L}$ & $\mathrm{P}$ & 2.558 & 0.391 & 0.621 & 91.8 & 0 & 2.526 & 0.637 & 93.0 & 2.526 & 0.637 & 93.0 \\
\hline 20402 & $186 \mathrm{PSCL}$ & L22 & L483 & $\mathrm{L}$ & $\mathrm{N}$ & 2.494 & 0.401 & 0.653 & 94.2 & 0 & & & & & & \\
\hline 20402 & $186 \mathrm{PSCL}$ & L22 & L483 & $\mathrm{T}$ & $\mathrm{P}$ & 2.212 & 0.452 & 0.526 & 27.1 dead & & 23.833 & 0.292 & 42.4 & & & \\
\hline 20402 & $186 \mathrm{PSCL}$ & L22 & L483 & $\mathrm{T}$ & $\mathrm{N}$ & 45.455 & 0.022 & 0.058 & 57.7 dead & & & & & & & \\
\hline 20402 & $186 \mathrm{PSCL}$ & L22 & L483 & $\mathrm{V}$ & $P$ & 1.845 & 0.542 & 0.647 & 85.0 & 1 & 1.866 & 0.665 & 86.6 & 1.866 & 0.665 & 86.6 \\
\hline 20402 & $186 \mathrm{PSCL}$ & L22 & L483 & V & $\mathrm{N}$ & 1.887 & 0.530 & 0.682 & 88.2 & 1 & & & & & & \\
\hline 20402 & $186 \mathrm{PSCL}$ & L22 & L483 & $\mathrm{L}$ & $\mathrm{P}$ & 2.571 & 0.389 & 0.625 & 92.5 & 0 & 2.532 & 0.629 & 93.1 & 2.425 & 0.604 & 90.0 \\
\hline 20402 & $186 \mathrm{PSCL}$ & L22 & L483 & $\mathrm{L}$ & $\mathrm{N}$ & 2.494 & 0.401 & 0.632 & 93.6 & 0 & & & & & & \\
\hline 20402 & $186 \mathrm{PSCL}$ & L22 & L483 & $\mathrm{T}$ & $\mathrm{P}$ & 2.347 & 0.426 & 0.574 & 86.0 & 0 & 2.318 & 0.579 & 87.0 & & & \\
\hline 20402 & $186 \mathrm{PSCL}$ & L22 & L483 & $\mathrm{T}$ & $\mathrm{N}$ & 2.288 & 0.437 & 0.584 & 88.0 & 0 & & & & & & \\
\hline 20418 & 188PSCL & L22 & L644 & V & $\mathrm{P}$ & 1.890 & 0.529 & 0.811 & 93.0 & 2 & 1.867 & 0.813 & 92.6 & 1.867 & 0.813 & 92.6 \\
\hline 20418 & 188PSCL & L22 & L644 & V & $\mathrm{N}$ & 1.844 & 0.542 & 0.815 & 92.2 & 2 & & & & & & \\
\hline 20418 & 188PSCL & L22 & L644 & $\mathrm{L}$ & $\mathrm{P}$ & 1.926 & 0.519 & 0.781 & 93.2 & 2 & 1.947 & 0.785 & 93.7 & 1.893 & 0.801 & 93.2 \\
\hline 20418 & 188PSCL & L22 & L644 & $\mathrm{L}$ & $\mathrm{N}$ & 1.967 & 0.508 & 0.789 & 94.2 & 2 & & & & & & \\
\hline 20418 & 188PSCL & L22 & L644 & $\mathrm{T}$ & $\mathrm{P}$ & 1.855 & 0.539 & 0.824 & 93.5 & 2 & 1.839 & 0.816 & 92.7 & & & \\
\hline 20418 & $188 \mathrm{PSCL}$ & L22 & L644 & $\mathrm{T}$ & $\mathrm{N}$ & 1.823 & 0.549 & 0.809 & 92.0 & 2 & & & & & & \\
\hline 20314 & $189 \mathrm{PSCL}$ & L22 & L1487 & V & $\mathrm{P}$ & 2.127 & 0.470 & 0.748 & 95.0 & 1 & 2.134 & 0.755 & 95.7 & 2.134 & 0.755 & 95.t \\
\hline 20314 & $189 \mathrm{PSCL}$ & L22 & L1487 & V & $\mathrm{N}$ & 2.141 & 0.467 & 0.761 & 96.4 & 1 & & & & & & \\
\hline 20314 & 189PSCL & L22 & L1487 & $\mathrm{L}$ & $\mathrm{P}$ & 2.058 & 0.486 & 0.800 & 95.9 & 1 & 1.994 & 0.777 & 94.0 & 2.102 & 0.731 & 93.6 \\
\hline 20314 & 189PSCL & L22 & L1487 & $\mathrm{L}$ & $\mathrm{N}$ & 1.929 & 0.519 & 0.753 & 92.0 & 2 & & & & & & \\
\hline 20314 & $189 \mathrm{PSCL}$ & L22 & L1487 & $\mathrm{T}$ & $\mathrm{P}$ & 2.210 & 0.453 & 0.658 & 90.9 & 0 & 2.211 & 0.685 & 93.3 & & & \\
\hline 20314 & 189PSCL & L22 & L1487 & $\mathrm{T}$ & $\mathrm{N}$ & 2.212 & 0.452 & 0.711 & 95.7 & 1 & & & & & & \\
\hline
\end{tabular}


TABLE 3: LOCAL EARTHQUAKES RECORDED ON THE SEATTLE SHIPS ARRAY

(Bold, italics indicate events shown in figures 7-14)

\begin{tabular}{|c|c|c|c|c|c|c|c|c|c|}
\hline Julian day & year & mo & day & time & $\begin{array}{c}\text { latitude } \\
\text { (degrees } \\
\text { north) }\end{array}$ & $\begin{array}{l}\text { longitude } \\
\text { (degrees) }\end{array}$ & depth $(\mathrm{km})$ & $\begin{array}{c}\text { mag } \\
\text { (coda) }\end{array}$ & location \\
\hline 26 & 2002 & 1 & 26 & $14: 01: 46$ & 47.1305 & -122.1325 & 8.5 & 2.4 & near Enumclaw \\
\hline 28 & 2002 & 1 & 28 & $1: 57: 09$ & 47.5197 & -122.8180 & 19.4 & 2.1 & near Bremerton \\
\hline 30 & 2002 & 1 & 30 & $4: 13: 13$ & 47.6905 & -121.9472 & 26 & 2.1 & near Carnation \\
\hline 31 & 2002 & 1 & 31 & $12: 42: 24$ & 46.8178 & -121.9673 & 6.9 & 2.1 & W. Rainier seismic zone \\
\hline 36 & 2002 & 2 & 5 & 6:21:34 & 47.7050 & -122.0508 & 19.3 & 1.5 & near Duvall \\
\hline 43 & 2002 & 2 & 12 & 19:16:41 & 48.4133 & -122.2857 & 18.6 & 3 & near Mt. Vernon \\
\hline 44 & 2002 & 2 & 13 & $7: 15: 45$ & 46.0070 & -122.7150 & 19 & 2.2 & near Longview \\
\hline 50 & 2002 & 2 & 19 & 18:07:20 & 46.8682 & -121.7560 & 1.4 & 2.5 & Mt. Ranier \\
\hline 50 & 2002 & 2 & 19 & $18: 42: 29$ & 46.8587 & -121.7530 & 0 & 3.2 & Mt. Ranier \\
\hline 52 & 2002 & 2 & 21 & $9: 48: 37$ & 46.1368 & -120.5138 & 16.8 & 2 & near Yakima \\
\hline 53 & 2002 & 2 & 22 & 9:39:35 & 47.3895 & -121.8152 & 20.4 & 1.7 & near North Bend \\
\hline 54 & 2002 & 2 & 23 & $14: 28: 56$ & 48.9300 & -123.0600 & 19 & 2.3 & Point Roberts, WA \\
\hline 58 & 2002 & 2 & 27 & $12: 06: 43$ & 47.5228 & -122.7527 & 21.2 & 1.6 & near Bremerton \\
\hline 59 & 2002 & 2 & 28 & $9: 46: 23$ & 46.9687 & -121.8935 & 8.2 & 1.6 & W. Rainier seismic zone \\
\hline 59 & 2002 & 2 & 28 & $12: 00: 51$ & 46.9722 & -121.9028 & 8.4 & 1.6 & W. Rainier seismic zone \\
\hline 59 & 2002 & 2 & 28 & $17: 07: 34$ & 48.3930 & -122.9047 & 49.4 & 2.3 & near Friday Harbor \\
\hline 60 & 2002 & 3 & 1 & $11: 11: 25$ & 47.5222 & -122.7492 & 21.9 & 1.3 & near Bremerton \\
\hline 61 & 2002 & 3 & 2 & $3: 09: 37$ & 48.3488 & -122.3195 & 11.6 & 1.7 & near Mt. Vernon \\
\hline 61 & 2002 & 3 & 2 & 9:50:37 & 47.9060 & -122.2117 & 21.3 & 2 & near Everett \\
\hline 63 & 2002 & 3 & 4 & $10: 49: 11$ & 46.8833 & -121.9080 & 11.7 & 2.1 & W. Rainier seismic zone \\
\hline 64 & 2002 & 3 & 5 & 18:16:05 & 47.4147 & -122.7047 & 28 & 1.8 & near Bremerton \\
\hline 64 & 2002 & 3 & 5 & $20: 35: 19$ & 47.0693 & -122.4992 & 17.3 & 1.4 & near Tacoma \\
\hline 68 & 2002 & 3 & 9 & $21: 16: 28$ & 48.3120 & -123.1175 & 20.9 & 1.8 & near Victoria, BC \\
\hline 69 & 2002 & 3 & 10 & $11: 04: 11$ & 47.5305 & -121.6392 & 15.3 & 1.9 & near North Bend \\
\hline 70 & 2002 & 3 & 11 & $0: 43: 51$ & 47.5070 & -122.7393 & 22.7 & 1.7 & near Bremerton \\
\hline 70 & 2002 & 3 & 11 & $2: 48: 45$ & 46.8507 & -119.7433 & 2.3 & 2.9 & near Vantage, WA \\
\hline
\end{tabular}




\begin{tabular}{|c|c|c|c|c|c|c|c|c|c|}
\hline 75 & 2002 & 3 & 16 & $1: 08: 56$ & 46.8527 & -121.9737 & 9 & 1.8 & W. Rainier \\
\hline 83 & 2002 & 3 & 24 & 11:05:12 & 47.4618 & -122.053 & 19.8 & 2.3 & Maple Valley \\
\hline 85 & 2002 & 3 & 26 & $8: 11: 46$ & 47.2577 & -122.809 & 8.6 & 1.9 & Olympia \\
\hline 85 & 2002 & 3 & 26 & 12:34:02 & 47.2855 & -122.216 & 18.1 & 2.5 & Tacoma \\
\hline 85 & 2002 & 3 & 26 & $12: 37: 10$ & 47.289 & -122.226 & 18.2 & 2.1 & Tacoma \\
\hline 88 & 2002 & 3 & 29 & $8: 53: 25$ & 47.4265 & -122.826 & 16 & 1.7 & Bremerton \\
\hline 94 & 2002 & 4 & 4 & 13:10:01 & 47.4758 & -122.895 & 19.8 & 1.8 & Bremerton \\
\hline 96 & 2002 & 4 & 6 & 10:00:28 & 47.4333 & -122.365 & 24.4 & 1.7 & Seattle \\
\hline 99 & 2002 & 4 & 9 & $17: 51: 56$ & 48.615 & -123.087 & 8.8 & 1.5 & Friday Harbor \\
\hline 100 & 2002 & 4 & 10 & $7: 38: 23$ & 47.6667 & -120.119 & 0.6 & 2.9 & Entiat \\
\hline 100 & 2002 & 4 & 10 & $22: 26: 38$ & 46.8698 & -121.936 & 10.9 & 2.2 & Rainier seismic zone \\
\hline 102 & 2002 & 4 & 12 & 5:45:12 & 47.3298 & -123.205 & 43.5 & 2.7 & Olympia \\
\hline 104 & 2002 & 4 & 14 & $22: 50: 31$ & 47.6968 & -122.013 & 20.1 & 1.2 & Duvall \\
\hline 105 & 2002 & 4 & 15 & $5: 23: 09$ & 47.6407 & -122.497 & 26 & 1.5 & Bremerton \\
\hline 106 & 2002 & 4 & 16 & $13: 38: 57$ & 48.4815 & -121.833 & 0 & 2.2 & Concrete \\
\hline 110 & 2002 & 4 & 20 & $10: 30: 50$ & 48.0063 & -121.544 & 3.8 & 2 & Darrington \\
\hline 112 & 2002 & 4 & 22 & $10: 38: 51$ & 47.2953 & -122.299 & 20.6 & 2.2 & Tacoma \\
\hline 116 & 2002 & 4 & 26 & $19: 44: 17$ & 47.6897 & -121.989 & 14.7 & 1.8 & Duvall \\
\hline 117 & 2002 & 4 & 27 & $20: 11: 28$ & 47.5915 & -122.347 & 55.5 & 1.9 & Seattle \\
\hline 119 & 2002 & 4 & 29 & 14:05:02 & 47.774 & -121.852 & 6.2 & 2 & Duvall \\
\hline 121 & 2002 & 5 & 1 & $6: 37: 30$ & 47.7523 & -121.859 & 4 & 1.6 & Duvall \\
\hline 121 & 2002 & 5 & 1 & 9:09:45 & 48.458 & -119.556 & 3.7 & 2.9 & Okanogan \\
\hline 122 & 2002 & 5 & 2 & $9: 26: 31$ & 47.8492 & -122.782 & 24.5 & 1.8 & Poulsbo \\
\hline 123 & 2002 & 5 & 3 & $0: 36: 47$ & 45.055 & -122.51 & 21.7 & 2.4 & Canby OR \\
\hline 124 & 2002 & 5 & 4 & 13:13:50 & 47.5847 & -122.441 & 25.9 & 1.5 & Seattle \\
\hline 125 & 2002 & 5 & 5 & $0: 36: 58$ & 47.3795 & -122.362 & 18.9 & 2.2 & Tacoma \\
\hline 125 & 2002 & 5 & 5 & $16: 40: 43$ & 48.2807 & -122.213 & 12.1 & 2 & Mt. Vernon \\
\hline 126 & 2002 & 5 & 6 & $4: 47: 53$ & 45.3245 & -121.686 & 5 & 2.5 & Mt. Hood \\
\hline 126 & 2002 & 5 & 6 & $11: 21: 58$ & 45.3297 & -121.688 & 5.3 & 2.8 & Mt. Hood \\
\hline 126 & 2002 & 5 & 6 & $11: 28: 47$ & 47.3863 & -122.07 & 6.8 & 1.5 & Maple Valley \\
\hline 126 & 2002 & 5 & 6 & 13:08:27 & 45.3295 & -121.688 & 4.7 & 2.5 & Mt. Hood \\
\hline 127 & 2002 & 5 & 7 & $18: 23: 39$ & 47.6458 & -122.758 & 24.9 & 1.5 & Bremerton \\
\hline 131 & 2002 & 5 & 11 & $9: 26: 39$ & 47.0207 & \begin{tabular}{|c|}
-122.006 \\
\end{tabular} & 15.4 & 1.4 & Enumclaw \\
\hline
\end{tabular}




\begin{tabular}{|r|r|r|r|r|r|r|r|l|l|}
\hline 131 & 2002 & 5 & 11 & $19: 10: 01$ & 47.7962 & -122.773 & 23 & 2.3 & Poulsbo \\
\hline $\mathbf{1 3 3}$ & $\mathbf{2 0 0 2}$ & $\mathbf{5}$ & $\mathbf{1 3}$ & $\mathbf{2 1 : 0 0 : 3 8}$ & $\mathbf{4 8 . 3 9 9 5}$ & $\mathbf{- 1 2 3 . 4 6 1}$ & $\mathbf{4 2 . 1}$ & $\mathbf{2 . 7}$ & Victoria, BC \\
\hline 134 & 2002 & 5 & 14 & $17: 13: 10$ & 47.8505 & -123.06 & 47.7 & 2.1 & Poulsbo \\
\hline 134 & 2002 & 5 & 14 & $22: 50: 19$ & 47.0095 & -122.006 & 15.3 & 1.9 & Enumclaw \\
\hline $\mathbf{1 3 9}$ & $\mathbf{2 0 0 2}$ & $\mathbf{5}$ & $\mathbf{1 9}$ & $\mathbf{1 7 : 0 5 : 2 7}$ & $\mathbf{4 8 . 3}$ & $\mathbf{- 1 2 2 . 1 9 5}$ & $\mathbf{1 3 . 7}$ & $\mathbf{2 . 7}$ & Mt. Vernon \\
\hline 140 & 2002 & 5 & 20 & $10: 44: 38$ & 47.7783 & -122.864 & 20.2 & 2 & Poulsbo \\
\hline 140 & 2002 & 5 & 20 & $12: 07: 35$ & 47.7708 & -122.849 & 20.5 & 1.4 & Poulsbo \\
\hline 144 & 2002 & 5 & 24 & $11: 17: 29$ & 48.0527 & -122.618 & 29.5 & 1 & Everett \\
\hline 147 & 2002 & 5 & 27 & $19: 33: 38$ & 46.9300 & -121.9600 & 13.1 & 2.4 & Mt. Rainier \\
\hline
\end{tabular}

TABLE 4: LOCAL BLASTS RECORDED ON THE SEATTLE SHIPS ARRAY

\begin{tabular}{|l|l|l|l|l|l|l|l|l|l|}
\hline Julian day & year & month & day & UTC time & $\begin{array}{l}\text { latitude } \\
\text { (degrees } \\
\text { north) }\end{array}$ & $\begin{array}{l}\text { longitude } \\
\text { (degrees) }\end{array}$ & depth (km) & $\begin{array}{l}\text { magnitude location } \\
\text { (coda) }\end{array}$ \\
\hline 50 & 2002 & 2 & 19 & $23: 14: 50$ & 46.7027 & -122.7753 & 0 & 3.2 & Centralia blast \\
\hline 52 & 2002 & 2 & 21 & $22: 55: 05$ & 46.6927 & -122.7748 & 0 & 3 & Centralia blast \\
\hline 54 & 2002 & 2 & 23 & $1: 46: 56$ & 47.8290 & -122.2172 & 0 & 1.7 & near Snohomish \\
\hline 54 & 2002 & 2 & 23 & $14: 28: 55$ & 48.9478 & -123.0513 & 0 & 2.3 & near Vancouver, BC \\
\hline 56 & 2002 & 2 & 25 & $21: 52: 55$ & 46.6975 & -122.7897 & 0 & 3.3 & Centralia blast \\
\hline 57 & 2002 & 2 & 26 & $22: 59: 09$ & 46.7025 & -122.7643 & 0 & 3.5 & Centralia blast \\
\hline 59 & 2002 & 2 & 28 & $22: 09: 31$ & 46.7013 & -122.7640 & 0 & 3.2 & Centralia blast \\
\hline 60 & 2002 & 3 & 1 & $23: 25: 39$ & 46.6933 & -122.7872 & 0 & 3.2 & Centralia blast \\
\hline 64 & 2002 & 3 & 5 & $22: 54: 06$ & 46.6995 & -122.7642 & 0 & 3.3 & Centralia blast \\
\hline 65 & 2002 & 3 & 6 & $23: 06: 49$ & 46.7412 & -122.7753 & 0 & 2.9 & Centralia blast \\
\hline 66 & 2002 & 3 & 7 & $21: 03: 07$ & 46.7027 & -122.7663 & 0 & 3.3 & Centralia blast \\
\hline 70 & 2002 & 3 & 11 & $22: 56: 06$ & 46.7015 & -122.7680 & 0 & 3 & Centralia blast \\
\hline 73 & 2002 & 3 & 14 & $23: 08: 57$ & 46.7032 & -122.7700 & 0 & 3.1 & Centralia blast \\
\hline 74 & 2002 & 3 & 15 & $22: 56: 05$ & 46.7337 & -122.7745 & 0 & 2.7 & Centralia blast \\
\hline 77 & 2002 & 3 & 18 & $23: 06: 53$ & 46.7095 & -122.7743 & 0 & 2.9 & Centralia blast \\
\hline 78 & 2002 & 3 & 19 & $23: 10: 30$ & 46.7352 & -122.78 & 0 & 2.7 & Centralia blast \\
\hline
\end{tabular}




\begin{tabular}{|c|c|c|c|c|c|c|c|c|c|}
\hline 79 & 2002 & 3 & 20 & 23:53:40 & 46.7163 & -122.727 & 0 & 3.1 & Centralia blast \\
\hline 80 & 2002 & 3 & 21 & $22: 07: 27$ & 46.7045 & -122.765 & 0 & 3.1 & Centralia blast \\
\hline 84 & 2002 & 3 & 25 & $22: 51: 11$ & 46.6997 & -122.772 & 0 & 3.2 & Centralia blast \\
\hline 85 & 2002 & 3 & 26 & $22: 44: 07$ & 46.7068 & -122.759 & 0 & 3.1 & Centralia blast \\
\hline 86 & 2002 & 3 & 27 & 22:19:22 & 46.7037 & -122.763 & 0 & 3.1 & Centralia blast \\
\hline 93 & 2002 & 4 & 3 & $22: 51: 34$ & 46.7052 & -122.776 & 0 & 3.1 & Centralia blast \\
\hline 94 & 2002 & 4 & 4 & $22: 16: 47$ & 46.706 & -122.763 & 0 & 3.3 & Centralia blast \\
\hline 95 & 2002 & 4 & 5 & $22: 49: 17$ & 46.701 & -122.765 & 0 & 3.3 & Centralia blast \\
\hline 98 & 2002 & 4 & 8 & $21: 21: 47$ & 46.7017 & -122.766 & 0 & 3.4 & Centralia blast \\
\hline 100 & 2002 & 4 & 10 & $21: 35: 00$ & 46.6998 & -122.772 & 0 & 3.2 & Centralia blast \\
\hline 101 & 2002 & 4 & 11 & $21: 51: 02$ & 46.7033 & -122.763 & 0 & 3.3 & Centralia blast \\
\hline 102 & 2002 & 4 & 12 & $21: 37: 54$ & 46.7027 & -122.773 & 0 & 3.2 & Centralia blast \\
\hline 105 & 2002 & 4 & 15 & $21: 26: 33$ & 46.7075 & -122.76 & 0 & 3.2 & Centralia blast \\
\hline 108 & 2002 & 4 & 18 & $20: 45: 33$ & 46.7382 & -122.806 & 0 & 3 & Centralia blast \\
\hline 109 & 2002 & 4 & 19 & $21: 19: 56$ & 46.7395 & -122.802 & 0 & 3.3 & Centralia blast \\
\hline 112 & 2002 & 4 & 22 & $21: 46: 16$ & 46.7377 & -122.812 & 0 & 3 & Centralia blast \\
\hline 121 & 2002 & 5 & 1 & $21: 47: 14$ & 46.7037 & -122.764 & 0 & 3.1 & Centralia blast \\
\hline 122 & 2002 & 5 & 2 & $21: 24: 27$ & 46.6992 & -122.762 & 0 & 3.1 & Centralia blast \\
\hline 123 & 2002 & 5 & 3 & $21: 31: 40$ & 46.705 & -122.767 & 0 & 3.3 & Centralia blast \\
\hline 124 & 2002 & 5 & 4 & $21: 37: 12$ & 46.6975 & -122.788 & 0 & 3.2 & Centralia blast \\
\hline 126 & 2002 & 5 & 6 & $20: 47: 26$ & 46.7045 & -122.781 & 0 & 2.7 & Centralia blast \\
\hline 127 & 2002 & 5 & 7 & $21: 51: 19$ & 46.7025 & -122.763 & 0 & 3.5 & Centralia blast \\
\hline 128 & 2002 & 5 & 8 & $21: 21: 18$ & 46.7057 & -122.765 & 0 & 3.5 & Centralia blast \\
\hline 129 & 2002 & 5 & 9 & $21: 47: 46$ & 46.7387 & -122.813 & 0 & 3.5 & Centralia blast \\
\hline 130 & 2002 & 5 & 10 & $21: 13: 18$ & 46.7387 & -122.817 & 0 & 3.2 & Centralia blast \\
\hline 131 & 2002 & 5 & 11 & $19: 56: 49$ & 46.704 & -122.768 & 0 & 3.2 & Centralia blast \\
\hline 132 & 2002 & 5 & 12 & 18:39:31 & 46.7145 & -122.764 & 0 & 3 & Centralia blast \\
\hline 133 & 2002 & 5 & 13 & $21: 21: 47$ & 46.7065 & -122.77 & 0 & 3.5 & Centralia blast \\
\hline 135 & 2002 & 5 & 15 & $21: 31: 32$ & 46.7 & -122.753 & 0 & 2.7 & Centralia blast \\
\hline 142 & 2002 & 5 & 22 & $20: 55: 15$ & 46.707 & -122.762 & 0 & 3.2 & Centralia blast \\
\hline 143 & 2002 & 5 & 23 & $21: 02: 10$ & 46.701 & -122.763 & 0 & 3 & Centralia blast \\
\hline 144 & 2002 & 5 & 24 & $21: 07: 56$ & 46.7002 & -122.768 & 0 & 3.3 & Centralia blast \\
\hline
\end{tabular}


TABLE 5: TELESEISMS RECORDED ON THE SEATTLE SHIPS ARRAY

(Bold, italics indicate events shown in figures 15-20)

\begin{tabular}{|c|c|c|c|c|c|c|c|c|c|c|}
\hline $\begin{array}{l}\text { Jul } \\
\text { day }\end{array}$ & $\mathrm{yr}$ & mo & day & UTC time & lat (deg) & long (deg) & $\begin{array}{c}\text { depth } \\
(\mathrm{km})\end{array}$ & $\begin{array}{c}\text { mag (mb } \\
\text { or MS) }\end{array}$ & $\begin{array}{l}\text { epicentral } \\
\text { dist (deg) }\end{array}$ & location \\
\hline 27 & 2002 & 1 & 27 & $7: 09: 14$ & 39.288 & 142.327 & 49 & 5.3 & 65 & Japan \\
\hline 28 & 2002 & 1 & 28 & $13: 50: 29$ & 49.381 & 155.594 & 33 & 6.1 & 52 & Kuril Islands \\
\hline 28 & 2002 & 1 & 28 & $15: 09: 55$ & -15.3040 & -173.2250 & 33 & 6.1 & 78 & Tonga Islands \\
\hline 30 & 2002 & 1 & 30 & $8: 42: 02$ & 18.1990 & -95.6910 & 106 & 5.6 & 37 & Veracruz, Mexico \\
\hline 30 & 2002 & 1 & 30 & 12:58:19 & -6.2460 & 150.8370 & 33 & 6 & 93 & New Britain region, New Guinea \\
\hline 31 & 2002 & 1 & 31 & $16: 27: 16$ & -12.6650 & 169.5370 & 626 & 5.3 & 85 & Santa Cruz Islands region \\
\hline 32 & 2002 & 2 & 1 & $21: 55: 20$ & 45.5440 & 136.6560 & 353 & 6.1 & 64 & Primorye, Russia \\
\hline 34 & 2002 & 2 & 3 & $7: 11: 28$ & 38.4900 & 31.3050 & 10 & 6.5 & 91 & Turkey \\
\hline 34 & 2002 & 2 & 3 & $9: 26: 43$ & 38.6280 & 30.8050 & 10 & 5.8 & 90 & Turkey \\
\hline 36 & 2002 & 2 & 5 & $13: 27: 24$ & -5.3430 & 151.3020 & 39 & 6.3 & 92 & New Britain region, New Guinea \\
\hline 37 & 2002 & 2 & 6 & $17: 18: 43$ & 61.2710 & -149.8690 & 45 & 4.9 & 21 & southern Alaska \\
\hline 40 & 2002 & 2 & 9 & $17: 19: 26$ & 61.3470 & -149.8810 & 45 & 4.9 & 21 & southern Alaska \\
\hline 40 & 2002 & 2 & 9 & $16: 56: 03$ & 46.1550 & 142.7700 & 300 & 5 & 60 & Sakhalin Island, Russia \\
\hline 40 & 2002 & 2 & 9 & $18: 49: 58$ & 43.447 & -126.7520 & 10 & & 5 & off coast of Oregon \\
\hline 41 & 2002 & 2 & 10 & $1: 47: 06$ & -55.9280 & -29.0860 & 192 & 5.6 & 129 & South Sandwich Islands \\
\hline 42 & 2002 & 2 & 11 & $3: 39: 32$ & -17.9970 & -178.4840 & 562 & 4.9 & 83 & Fiji \\
\hline 43 & 2002 & 2 & 12 & $3: 27: 23$ & 23.7130 & 121.5550 & 33 & 5.9 & 89 & Taiwan \\
\hline 43 & 2002 & 2 & 12 & $13: 44: 35$ & 36.5860 & 140.9190 & 33 & 5.6 & 68 & Honshu, Japan \\
\hline 44 & 2002 & 2 & 13 & $14: 17: 10$ & -12.7010 & 169.6200 & 600 & 4.8 & 85 & Santa Cruz Islands region \\
\hline 45 & 2002 & 2 & 14 & $1: 12: 21$ & 41.5260 & 141.9930 & 62 & 5.1 & 64 & Hokkaido, Japan \\
\hline 45 & 2002 & 2 & 14 & $23: 23: 13$ & 14.9750 & -92.4700 & 74 & 5.3 & 41 & Chiapas, Mexico \\
\hline 46 & 2002 & 2 & 15 & $1: 46: 38$ & -36.1540 & -100.2390 & 10 & 5.4 & 86 & Easter Island \\
\hline 49 & 2002 & 2 & 18 & $13: 52: 36$ & -21.0070 & -179.2120 & 625 & 4.8 & 86 & Fiji \\
\hline 50 & 2002 & 2 & 19 & $0: 35: 49$ & -3.8160 & 150.9480 & 33 & 6 & 91 & New Ireland, New Guinea \\
\hline 50 & 2002 & 2 & 19 & 12:33:24 & -56.7210 & -25.4840 & 33 & 5.4 & 131 & South Sandwich Islands \\
\hline 51 & 2002 & 2 & 20 & $3: 17: 16$ & 51.4480 & -130.6300 & 10 & 4.5 & 7 & Queen Charlotte Islands \\
\hline 51 & 2002 & 2 & 20 & 19:07:16 & -7.7090 & 31.9860 & 37 & 5.6 & 134 & Lake Tanganyika \\
\hline 52 & 2002 & 2 & 21 & $8: 57: 46$ & -31.5320 & -67.1480 & 120 & 5.2 & 93 & San Juan province, Argentina \\
\hline
\end{tabular}




\begin{tabular}{|c|c|c|c|c|c|c|c|c|c|c|}
\hline 52 & 2002 & 2 & 21 & 9:15:17 & 18.5920 & 145.4650 & 209 & 5 & 78 & Mariana Islands \\
\hline 53 & 2002 & 2 & 22 & $19: 32: 41$ & 32.3750 & -115.3520 & 10 & 5.5 & 16 & Baja California \\
\hline 54 & 2002 & 2 & 23 & 19:37:14 & -4.4700 & 152.0350 & 159 & 5.6 & 90 & New Britain region, New Guinea \\
\hline 56 & 2002 & 2 & 25 & $21: 19: 26$ & 60.5690 & -147.4440 & 33 & 4.8 & 19 & southern Alaska \\
\hline 59 & 2002 & 2 & 28 & $1: 50: 50$ & -5.6020 & 151.2460 & 45 & 6.4 & 92 & New Britain region, New Guinea \\
\hline 62 & 2002 & 3 & 3 & $7: 16: 19$ & -45.8250 & -75.8320 & 33 & 6 & 102 & southern Chile \\
\hline 62 & 2002 & 3 & 3 & 12:08:12 & 36.4710 & 70.4010 & 256 & 7.4 & 95 & Hindu Kush, Afghanistan (2 events) \\
\hline 63 & 2002 & 3 & 4 & $20: 21: 21$ & 28.4150 & 143.2950 & 33 & 5.5 & 72 & Bonin Islands, Japan \\
\hline 63 & 2002 & 3 & 4 & $20: 37: 14$ & 28.4030 & 143.2290 & 33 & 5.2 & 72 & Bonin Islands, Japan \\
\hline 64 & 2002 & 3 & 5 & 8:25:04 & 20.7050 & 145.1910 & 105 & 5.1 & 76 & Mariana Islands \\
\hline 64 & 2002 & 3 & 5 & $21: 16: 09$ & 6.1710 & 124.2840 & 31 & 7.5 & 101 & Mindanao, Philippines (21:48 also) \\
\hline 66 & 2002 & 3 & 7 & 0:07:07 & 47.9440 & 146.8920 & 442 & 5.6 & 57 & Kuril Islands \\
\hline 67 & 2002 & 3 & 8 & $18: 27: 53$ & 5.8460 & 124.2720 & 23 & 6 & 101 & Mindanao, Philippines \\
\hline 67 & 2002 & 3 & 8 & 19:08:25 & 5.9270 & 124.3700 & 33 & 5 & 101 & Mindanao, Philippines \\
\hline 68 & 2002 & 3 & 9 & 3:39:17 & -17.9450 & -178.9280 & 500 & 4.6 & 83 & Fiji Islands \\
\hline 68 & 2002 & 3 & 9 & 7:42:57| & 56.6600 & -159.6120 & 147 & 4.5 & 24 & Alaska Peninsula (7:43.00 also) \\
\hline 68 & 2002 & 3 & 9 & $12: 27: 11$ & -56.0810 & -27.4940 & 118 & 6 & 130 & South Sandwich Islands \\
\hline 70 & 2002 & 3 & 11 & $1: 46: 20$ & 30.6310 & 141.5610 & 33 & 5.8 & 72 & Honshu, Japan \\
\hline 73 & 2002 & 3 & 14 & 16:08:32 & 51.6920 & -173.1500 & 33 & 5.9 & 32 & Andreanof Islands, Aleutian Islands \\
\hline 75 & 2002 & 3 & 16 & $20: 50: 04$ & -6.2290 & 151.3870 & 56 & 5.6 & 92 & New Britain region, New Guinea \\
\hline 76 & 2002 & 3 & 17 & 3:37:18 & 0.6350 & 122.3340 & 72 & 5.8 & 106 & Minahassa Peninsula, Sulawesi \\
\hline 76 & 2002 & 3 & 17 & $3: 57: 47$ & 51.4640 & -173.2710 & 33 & 5.5 & 33 & Andreanof Islands, Aleutian Islands \\
\hline 76 & 2002 & 3 & 17 & 9:00:59 & 12.5050 & -87.9700 & 69 & 5 & 45 & Nicaragua \\
\hline 76 & 2002 & 3 & 17 & $17: 51: 47$ & -23.5890 & 178.8460 & 527 & 4.5 & 89 & Fiji \\
\hline 76 & 2002 & 3 & 17 & 19:33:34 & -45.1430 & 34.7600 & 10 & 6 & 164 & Prince Edward Islands \\
\hline 76 & 2002 & 3 & 17 & $20: 50: 32$ & -33.2370 & -179.7500 & 33 & 5.6 & 96 & Kermadec Islands \\
\hline 76 & 2002 & 3 & 17 & $21: 43: 30$ & -37.1150 & -179.7390 & 33 & 5.6 & 99 & North Island, New Zealand \\
\hline 76 & 2002 & 3 & 17 & $22: 13: 17$ & -37.1860 & -179.9080 & 33 & 5.5 & 99 & North Island, New Zealand \\
\hline 77 & 2002 & 3 & 18 & $3: 09: 57$ & -20.3530 & -68.8400 & 93 & 5.5 & 83 & Chile/Bolivia \\
\hline 77 & 2002 & 3 & 18 & $22: 24: 36$ & -4.8000 & -102.1610 & 33 & 5.3 & 55 & Sumatera, Indonesia \\
\hline 78 & 2002 & 3 & 19 & $5: 03: 47$ & 62.9220 & -151.4380 & 33 & 5.1 & 22 & central Alaska \\
\hline 78 & 2002 & 3 & 19 & $17: 32: 14$ & 60.419 & -153.716 & 177 & 4.5 & 22 & southern Alaska \\
\hline 78 & 2002 & 3 & 19 & $20: 06: 19$ & 22.238 & 143.613 & 140 & 4.9 & 76 & Volcano Islands, Japan \\
\hline
\end{tabular}




\begin{tabular}{|c|c|c|c|c|c|c|c|c|c|c|}
\hline 79 & 2002 & 3 & 20 & 4:00:21 & 30.5730 & 141.8800 & 33 & 5.9 & 71 & southeast Honshu, Japan \\
\hline 79 & 2002 & 3 & 20 & $11: 37: 43$ & 126.58 & -152.9940 & 90 & & 76 & southern Alaska \\
\hline 79 & 2002 & 3 & 20 & $11: 40: 20$ & -23.18 & -179.893 & 327 & 4.8 & 88 & south of Fiji Islands \\
\hline 79 & 2002 & 3 & 20 & $14: 29: 58$ & -3.4080 & 144.9290 & 33 & 5.2 & 94 & north coast of New Guinea \\
\hline 81 & 2002 & 3 & 22 & 9:10:14 & -3.1340 & 142.3090 & 33 & 5 & 96 & north coast of New Guinea \\
\hline 81 & 2002 & 3 & 22 & 9:22:33 & -3.1350 & 142.3370 & 33 & 5.2 & 96 & north coast of New Guinea \\
\hline 81 & 2002 & 3 & 22 & $12: 21: 10$ & -18.459 & 178.336 & 558 & 4.6 & 85 & Fiji Islands \\
\hline 81 & 2002 & 3 & 22 & $17: 36: 54$ & 4.5970 & 126.3340 & 33 & 5.5 & 101 & Talud Islands, Indonesia \\
\hline 82 & 2002 & 3 & 23 & $3: 06: 18$ & 12.054 & 142.922 & 33 & 4.9 & 84 & Mariana Islands \\
\hline 82 & 2002 & 3 & 23 & $3: 30: 16$ & -16.041 & -177.964 & 500 & 4.8 & 81 & Fiji \\
\hline 82 & 2002 & 3 & 23 & 5:15:51 & 1.3880 & 128.0410 & 117 & 5.7 & 102 & Indonesia \\
\hline 83 & 2002 & 3 & 24 & 13:19:50 & 43.364 & \begin{tabular}{|c|}
-126.58 \\
\end{tabular} & 10 & & 5 & offshore Oregon \\
\hline 83 & 2002 & 3 & 24 & $16: 42: 37$ & -57.7650 & -66.4890 & 10 & 5.6 & 115 & Drake Passage \\
\hline 83 & 2002 & 3 & 24 & $18: 48: 53$ & -23.88 & -66.489 & 214 & 4.6 & 87 & Jujuy province, Argentina \\
\hline 85 & 2002 & 3 & 26 & 3:45:49 & 23.466 & 124.063 & 33 & 6.4 & 87 & Ryukyu Island, Japan \\
\hline 85 & 2002 & 3 & 26 & $10: 15: 10$ & -18.85 & 169.145 & 130 & 5.1 & 90 & Vanuatu Islands (New Hebrides) \\
\hline 86 & 2002 & 3 & 27 & $3: 52: 50$ & 40.38 & -126.359 & 10 & 4 & 8 & offshore northern California \\
\hline 86 & 2002 & 3 & 27 & $12: 15: 24$ & 44.952 & 147.581 & 121 & 5.1 & 59 & Kuril Islands \\
\hline 87 & 2002 & 3 & 28 & $4: 56: 21$ & -20.336 & -68.13 & 122 & 6.3 & 84 & Chile/Bolivia border \\
\hline 87 & 2002 & 3 & 28 & $5: 48: 24$ & 22.559 & -45.006 & 10 & 5.5 & 65 & Mid Atlantic Ridge (05:50:37 also) \\
\hline 90 & 2002 & 3 & 31 & 6:52:50 & 24.439 & 122.201 & 33 & 7.1 & 88 & Taiwan \\
\hline 91 & 2002 & 4 & 1 & $8: 44: 22$ & 44.109 & -128.886 & 10 & 4.5 & 6 & offshore Oregon \\
\hline 91 & 2002 & 4 & 1 & $14: 11: 40$ & 43.332 & -126.245 & 10 & & 5 & offshore Oregon \\
\hline 91 & 2002 & 4 & 1 & 19:59:32 & -29.483 & \begin{tabular}{|c|}
-71.069 \\
\end{tabular} & 67 & 6.4 & 90 & central Chile \\
\hline 92 & 2002 & 4 & 2 & 17:09:58 & -49.566 & -116.024 & 10 & 5.6 & 97 & southern east Pacific rise \\
\hline 93 & 2002 & 4 & 3 & $23: 42: 13$ & 41.656 & 141.853 & 72 & 5.7 & 64 & Hokkaido, Japan \\
\hline 94 & 2002 & 4 & 4 & 4:29:11 & 50.765 & -129.879 & 10 & 4.5 & 6 & Vancouver Island \\
\hline 95 & 2002 & 4 & 5 & $2: 41: 12$ & -6.317 & 130.086 & 33 & 5.8 & 106 & Banda Sea \\
\hline 95 & 2002 & 4 & 5 & $23: 02: 29$ & -15.204 & -173.433 & 33 & 5.7 & 78 & Tonga Islands \\
\hline 97 & 2002 & 4 & 7 & $1: 41: 25$ & -60.966 & 154.818 & 10 & 6.2 & 127 & west of McQuarie Island \\
\hline 97 & 2002 & 4 & 7 & $12: 09: 41$ & -10.791 & 164.178 & 33 & 5.8 & 87 & Santa Cruz Islands region \\
\hline 98 & 2002 & 4 & 8 & $3: 48: 54$ & -50.999 & 139.263 & 10 & 6.2 & 130 & west Indian-Antarctic Ridge \\
\hline 100 & 2002 & 4 & 10 & 10:04:50 & -44.008 & -15.825 & 10 & 5.7 & 131 & south mid-Atlantic ridge \\
\hline
\end{tabular}




\begin{tabular}{|c|c|c|c|c|c|c|c|c|c|c|}
\hline 100 & 2002 & 4 & 10 & 10:09:21 & -20.797 & 169.232 & 33 & 5.9 & 92 & Vanuatu Islands \\
\hline 101 & 2002 & 4 & 11 & $21: 56: 56$ & -14.386 & 167.623 & 10 & 6.2 & 88 & Vanuatu Islands (also 4 min later) \\
\hline 102 & 2002 & 4 & 12 & 4:00:23 & 35.914 & 69.228 & 10 & 5.9 & 96 & Afghanistan \\
\hline 103 & 2002 & 4 & 13 & $15: 36: 00$ & 1.099 & 15.335 & 33 & 5.5 & 119 & northern Molucca sea \\
\hline 104 & 2002 & 4 & 14 & $2: 04: 28$ & 38.608 & 73.29 & 178 & 5.5 & 93 & Tajikistan \\
\hline 104 & 2002 & 4 & 14 & $4: 05: 23$ & 7.319 & 126.651 & 33 & 5.6 & 98 & Philippines \\
\hline 105 & 2002 & 4 & 15 & 3:52:07 & 13.142 & 143.76 & 123 & 5.4 & 83 & Mariana Islands \\
\hline 106 & 2002 & 4 & 16 & $13: 25: 25$ & 52.0470 & -170.0620 & 33 & 4.8 & 31 & Fox Island, Aleutians \\
\hline 108 & 2002 & 4 & 18 & $5: 02: 47$ & 16.9450 & -100.8160 & 33 & 6.3 & 35 & Guerrero, Mexico \\
\hline 108 & 2002 & 4 & 18 & $14: 17: 27$ & -60.7320 & -26.0840 & 33 & 5.8 & 133 & South Sandwich Islands \\
\hline 108 & 2002 & 4 & 18 & 16:08:36 & -27.5350 & -70.6000 & 62 & 6.7 & 88 & northern Chile \\
\hline 110 & 2002 & 4 & 20 & $10: 50: 45$ & 44.4670 & -73.6900 & 11 & 5 & 33 & New York (also 14 minutes later) \\
\hline 110 & 2002 & 4 & 20 & 15:59:57 & -16.4140 & 173.2350 & 33 & 6 & 86 & Fiji \\
\hline 111 & 2002 & 4 & 21 & $16: 37: 38$ & 43.5370 & -126.6840 & 10 & 4.1 & 5 & offshore Oregon \\
\hline 113 & 2002 & 4 & 23 & 15:05:32 & -12.4610 & 166.9300 & 218 & 5.6 & 87 & Santa Cruz Islands region \\
\hline 114 & 2002 & 4 & 24 & 7:08:16 & 51.1310 & -177.8820 & 33 & 5.2 & 35 & Andreanof Islands, Aleutian Islands \\
\hline 114 & 2002 & 4 & 24 & $10: 51: 50$ & 42.4100 & 21.4200 & 10 & 5.7 & 84 & northwest Balkan region \\
\hline 114 & 2002 & 4 & 24 & $11: 00: 00$ & -56.1620 & -122.0250 & 10 & 6.2 & 104 & east Pacific rise \\
\hline 116 & 2002 & 4 & 26 & $7: 15: 08$ & 53.6140 & 160.4770 & 33 & 5.8 & 47 & Kamchatka \\
\hline 116 & 2002 & 4 & 26 & 16:06:06 & 13.1450 & 144.5850 & 80 & 7.1 & 82 & Mariana Islands \\
\hline 118 & 2002 & 4 & 28 & $13: 23: 49$ & 24.2130 & 122.7090 & 33 & 5.4 & 87 & Taiwan \\
\hline 121 & 2002 & 5 & 1 & $14: 31: 37$ & 44.3600 & 129.3400 & 10 & 4.8 & 69 & offshore Oregon \\
\hline 123 & 2002 & 5 & 3 & 22:32:04 & -18.181 & -178.308 & 619 & 4.8 & 83 & Fiji \\
\hline 124 & 2002 & 5 & 4 & $7: 00: 48$ & -17.898 & -178.737 & 560 & 5.8 & 83 & Fiji \\
\hline 124 & 2002 & 5 & 4 & 12:51:38 & -23.072 & -64.492 & 87 & 5.6 & 88 & Argentina \\
\hline 127 & 2002 & 5 & 7 & $15: 16: 07$ & -19.033 & 168.665 & 36 & 5.9 & 91 & Vanuatu \\
\hline 127 & 2002 & 5 & 7 & $20: 36: 48$ & 44.19 & -128.921 & 10 & 4 & 6 & offshore Oregon \\
\hline 128 & 2002 & 5 & 8 & 5:26:00 & -17.948 & -174.573 & 128 & 5.4 & 81 & Tonga Islands \\
\hline 128 & 2002 & 5 & 8 & $11: 20: 36$ & 51.63 & 175.311 & 33 & 4.8 & 39 & Andreanof Islands, Aleutian Islands \\
\hline 128 & 2002 & 5 & 8 & 19:45:18 & 53.813 & 160.774 & 39 & 5.9 & 47 & Kamchatka \\
\hline 129 & 2002 & 5 & 9 & $23: 41: 31$ & 2.6460 & 128.3030 & 173 & 5.7 & 101 & Indonesia \\
\hline 131 & 2002 & 5 & 11 & $10: 43: 08$ & -10.424 & -78.506 & 47 & 5.7 & 70 & Peru \\
\hline 132 & 2002 & 5 & 12 & $1: 29: 35$ & 39.225 & 140.995 & 96 & 5.3 & 66 & Honshu, Japan \\
\hline
\end{tabular}




\begin{tabular}{|r|r|r|r|r|r|r|r|r|c|l|}
\hline 132 & 2002 & 5 & 12 & $23: 12: 53$ & -1.143 & 127.087 & 19 & 5.9 & 105 & Indonesia \\
\hline 133 & 2002 & 5 & 13 & $3: 32: 51$ & 50.46 & -130.26 & 10 & 4.3 & 6 & Vancouver Island \\
\hline 133 & 2002 & 5 & 13 & $19: 57: 23$ & 19.132 & 121.238 & 32 & 5.8 & 92 & Philippines \\
\hline 134 & 2002 & 5 & 14 & $5: 00: 29$ & 36.967 & -121.6 & 8 & 4.7 & 11 & Gilroy, CA \\
\hline 135 & 2002 & 5 & 15 & $3: 27: 39$ & -21.4 & -174.314 & 3 & 5.9 & 83 & Tonga Islands \\
\hline 135 & 2002 & 5 & 15 & $7: 06: 20$ & 43.41 & -127.076 & 10 & 5 & 5 & offshore Oregon \\
\hline 135 & 2002 & 5 & 15 & $17: 54: 48$ & 42.231 & -121.901 & 8 & 4.2 & 5 & Klamath Falls, Oregon \\
\hline 136 & 2002 & 5 & 16 & $14: 44: 25$ & 43.365 & -126.777 & 10 & 4.1 & 5 & offshore Oregon \\
\hline 137 & 2002 & 5 & 17 & $10: 40: 11$ & 48.193 & -27.816 & 14 & 5.7 & 59 & Mid Atlantic Ridge (Azores) \\
\hline 141 & 2002 & 5 & 21 & $6: 03: 00$ & 17.779 & -81.915 & 13 & 5.7 & 44 & Honduras \\
\hline 141 & 2002 & 5 & 21 & $20: 04: 17$ & 44.619 & 146.517 & 142 & 5.5 & 59 & Kuril Islands \\
\hline 141 & 2002 & 5 & 21 & $23: 45: 35$ & 14.126 & 144.954 & 119 & 5.4 & 81 & Mariana Islands \\
\hline 142 & 2002 & 5 & 22 & $18: 57: 19$ & -36.344 & -97.908 & 10 & 5.3 & 87 & Easter Island \\
\hline 143 & 2002 & 5 & 23 & $15: 52: 16$ & -30.649 & -71.15 & 39 & 6 & 91 & Chile \\
\hline 143 & 2002 & 5 & 23 & $22: 05: 55$ & -5.808 & 101.988 & 20 & 5.6 & 124 & Sumatera, Indonesia \\
\hline 144 & 2002 & 5 & 24 & $0: 23: 15$ & -31.868 & -70.882 & 52 & 5.7 & 92 & Chile \\
\hline
\end{tabular}




\section{TABLE 6. PASSCAL SEGY TRACE HEADER FORMAT}

\begin{tabular}{|c|c|}
\hline Byte \# & Description \\
\hline $1-4$ & Trace sequence number within data stream \\
\hline $5-8$ & Trace sequence number within reel (same as above) \\
\hline $9-12$ & Event number \\
\hline $13-16$ & Channel number $=1$ or 4 for the vertical component, 2 or 5 for the N-S horizontal component, 3 or 6 for the E-W horizontal component \\
\hline $29-30$ & Trace identification code $=1$ for seismic data \\
\hline $53-56$ & datumElevRec = UTM Easting $(\mathrm{m})$ \\
\hline $56-60$ & datumElevSource $=$ UTM Northing $(\mathrm{m})$ \\
\hline $69-70$ & Elevation constant $=1$ \\
\hline $71-72$ & Coordinate constant $=1$ \\
\hline $81-84$ & recLongOrX = receiver longitude $* 3600$ \\
\hline $85-88$ & recLatOrY = receiver latitude $* 3600$ \\
\hline $89-90$ & Coordinate units $=2$ for Lat $/$ Long \\
\hline $103-104$ & Low 2 bytes of the total shift in milliseconds \\
\hline $115-116$ & $\begin{array}{l}\text { Number of samples in this trace } \\
\quad \text { (note if equal } 32767 \text { see bytes } 229-232 \text { ) }\end{array}$ \\
\hline $117-118$ & $\begin{array}{l}\text { Sample interval in microsecs for this trace } \\
\text { (note if equal } 1 \text { see bytes } 201-204 \text { ) }\end{array}$ \\
\hline $119-120$ & Fixed gain flag = 1 \\
\hline $121-122$ & Gain of amplifier \\
\hline $157-158$ & Year data recorded \\
\hline $159-160$ & Day of year \\
\hline $161-162$ & Hour of day (24 hour clock) \\
\hline $163-164$ & Minute of hour \\
\hline $165-166$ & Second of minute \\
\hline $167-168$ & Time basis code: $1=$ local $2=$ GMT $3=$ other \\
\hline $181-186^{*}$ & Station Name (6 chars) \\
\hline $187-194^{*}$ & Sensor Serial number ( 7 chars +1 for termination) \\
\hline $195-198 *$ & Channel Name code ( 3 chars +1 for termination) \\
\hline $199-200 *$ & Extra bytes ( 2 chars $)$ \\
\hline $201-204 *$ & Sample interval in microsecs as a 32 bit integer \\
\hline $205-206^{*}$ & Data format flag: $0=16$ bit integer $1=32$ bit integer \\
\hline $207-208 *$ & Milliseconds of second for first sample \\
\hline
\end{tabular}




\begin{tabular}{|ll|}
\hline $209-210^{*}$ & Trigger time year \\
$211-212^{*}$ & Trigger time Julian day \\
$213-214^{*}$ & Trigger time hour \\
$215-216^{*}$ & Trigger time minutes \\
$217-218^{*}$ & Trigger time seconds \\
$219-220^{*}$ & Trigger time milliseconds \\
$221-224^{*}$ & Scale factor (IEEE 32 bit float) \\
& $\quad$ (true amplitude = (data value)*(scale factor)/gain \\
$225-226^{*}$ & Instrument Serial Number \\
$229-232^{*}$ & Number of Samples as a 32 bit integer \\
$233-236^{*}$ & Max value in counts. \\
$237-240^{*}$ & Min value in counts. \\
\hline
\end{tabular}

*Header values not specified in the standard SEGY format 


\section{FIGURES}

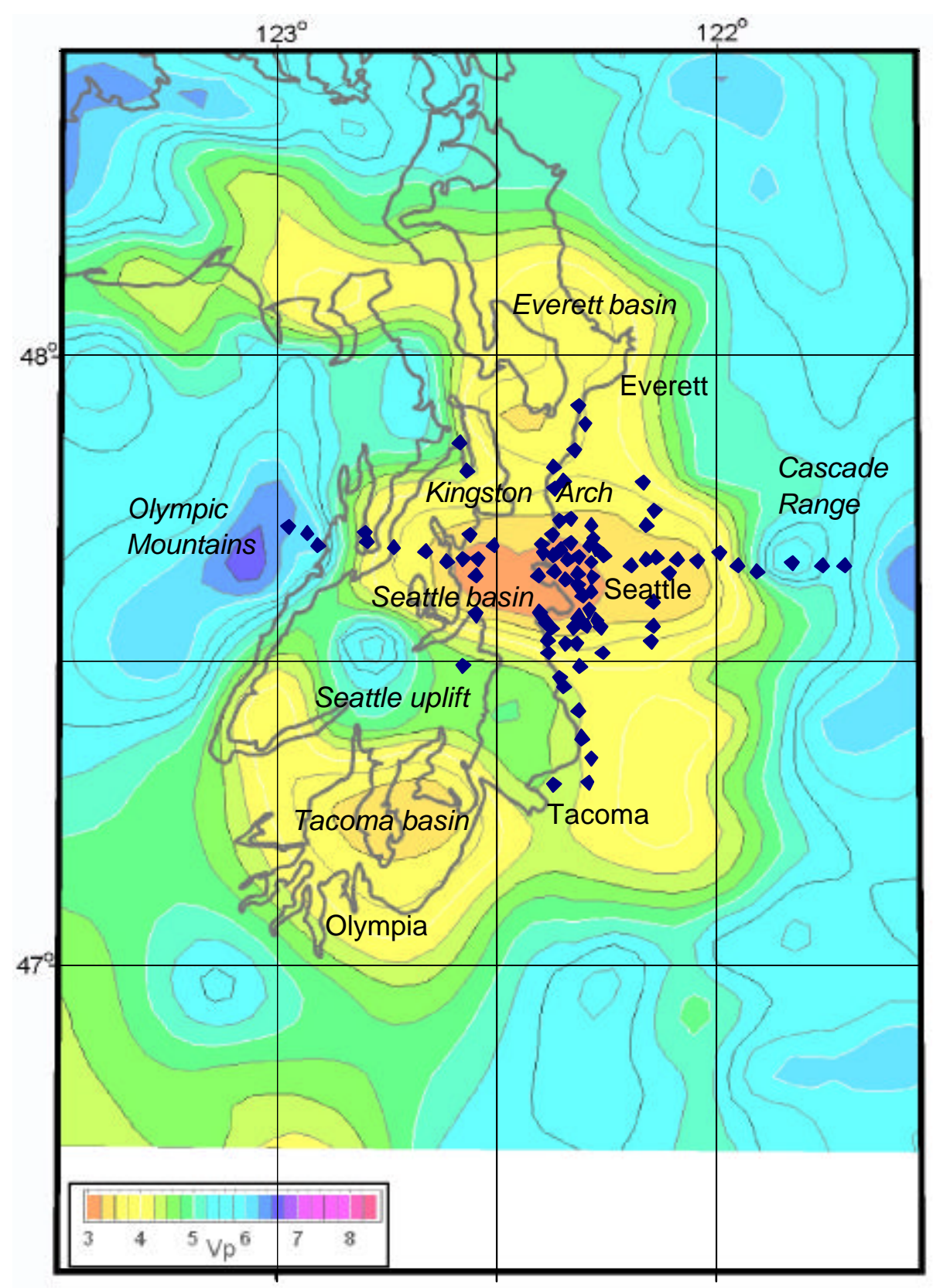

Figure 1: Stations locations superimposed on a tomography map. The colored background shows the speed of sound at $2.5 \mathrm{~km}$ depth derived from a regional tomographic study (VanWagoner et al., 2002). The blue dots are the locations of the Seattle SHIPS seismometer sites. The sites span the basin in both the north-south and east-west directions, and provide some 3-dimensional control over the basin. 


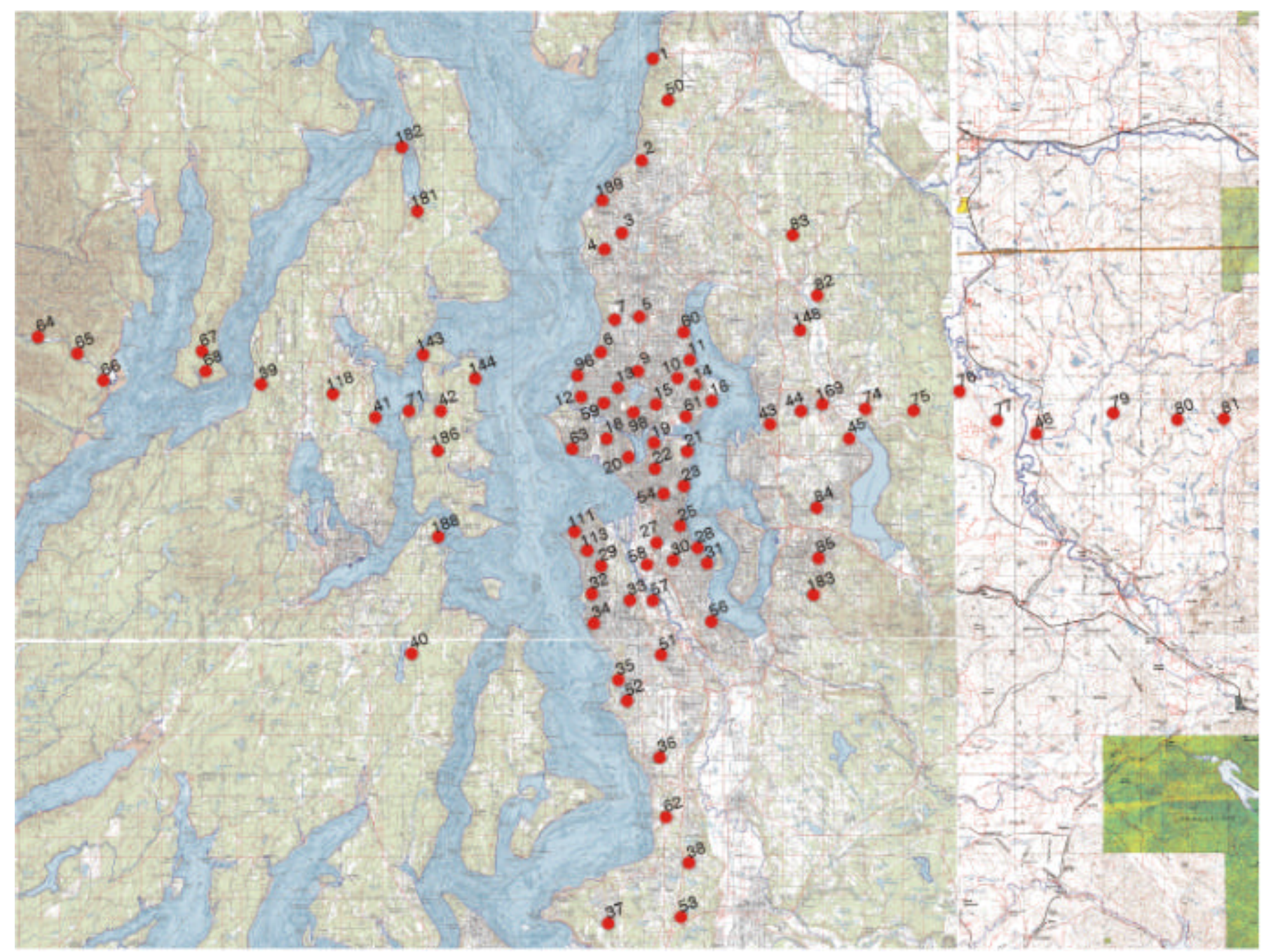

Figure 2: Topographic map showing the stations occupied during the 2002 Seattle Ships experiment. 




Figure 3: Instrumentation placement at each site. The DAS, car battery, and battery charger were placed in a plastic storage container outside of the house or garage. The sensor was buried about $2 \mathrm{~m}$ from the storage container, and the GPS antenna (or external clock) was placed wherever it would have an unobstructed view of the sky. Electrical power was provided to the battery charger from an outdoor power outlet. 


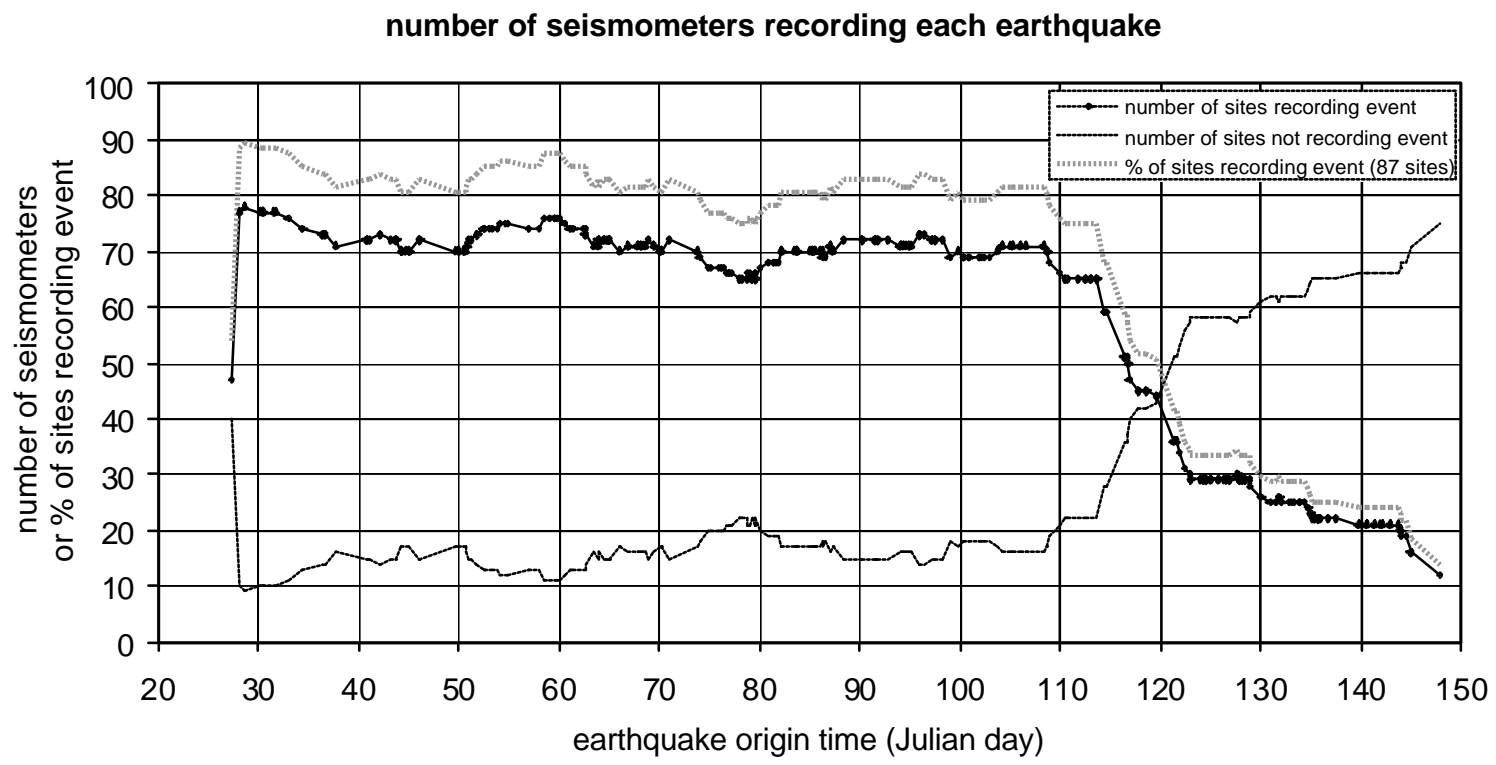

Figure 4: Number of seismometers recording each event. Each data point represents one event (tables 3-5) plotted by origin time. The vertical scale shows the number of instruments that recorded each event. We began removing instruments at day 108, resulting in a decrease in the number of instruments recording events after day 108. The dashed line shows the number of sites that did not record the event because of malfunctioning or missing instruments. The gray line shows the percentage of sites that recorded each event. 


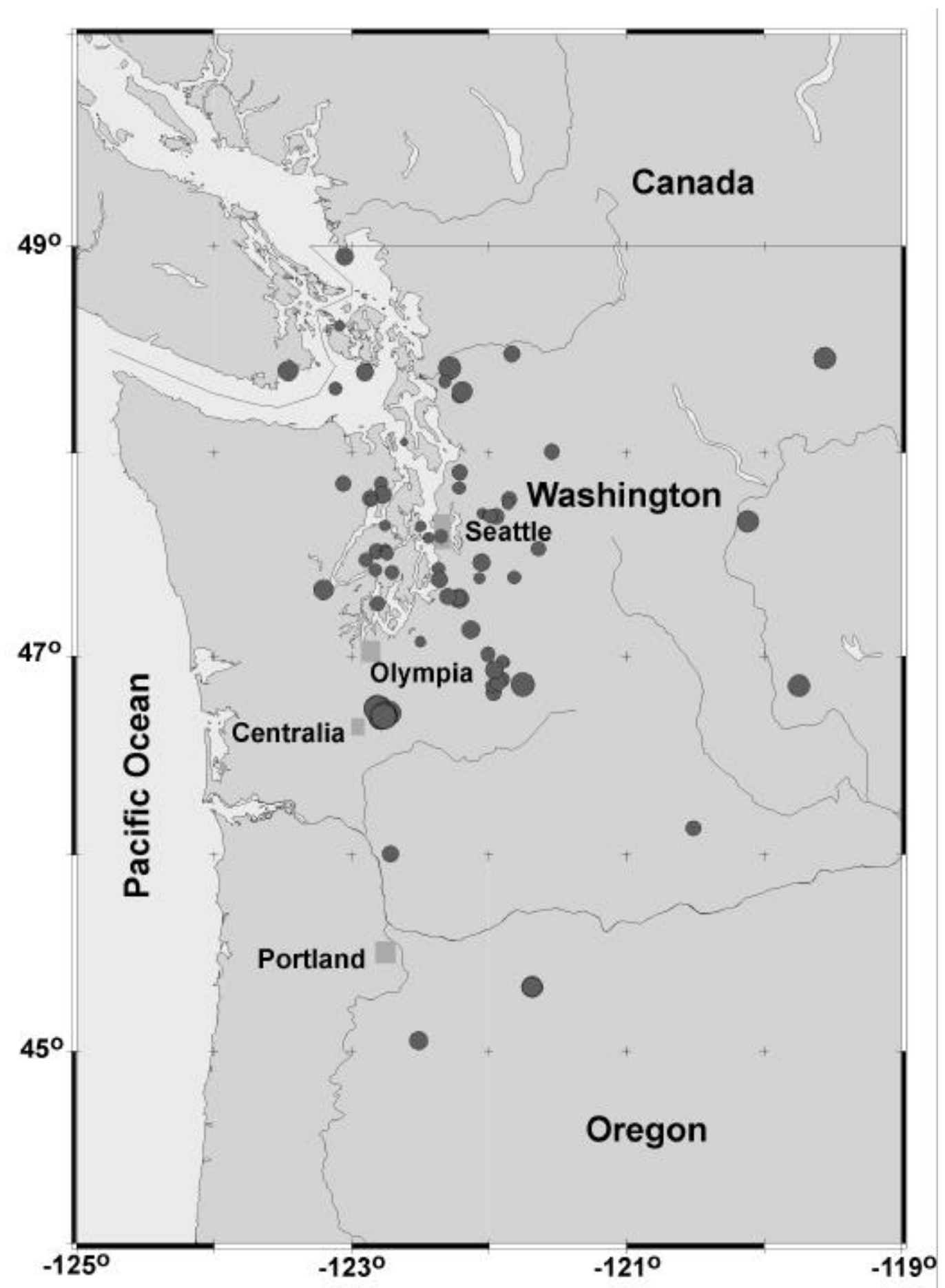

Figure 5: Map showing the locations of the local earthquakes recorded on the Seattle SHIPS array, with the size of the dot proportional to the earthquake magnitude. The collection of magnitude 3 events near Centralia are mine blasts; nearly all of the other events are earthquakes. 


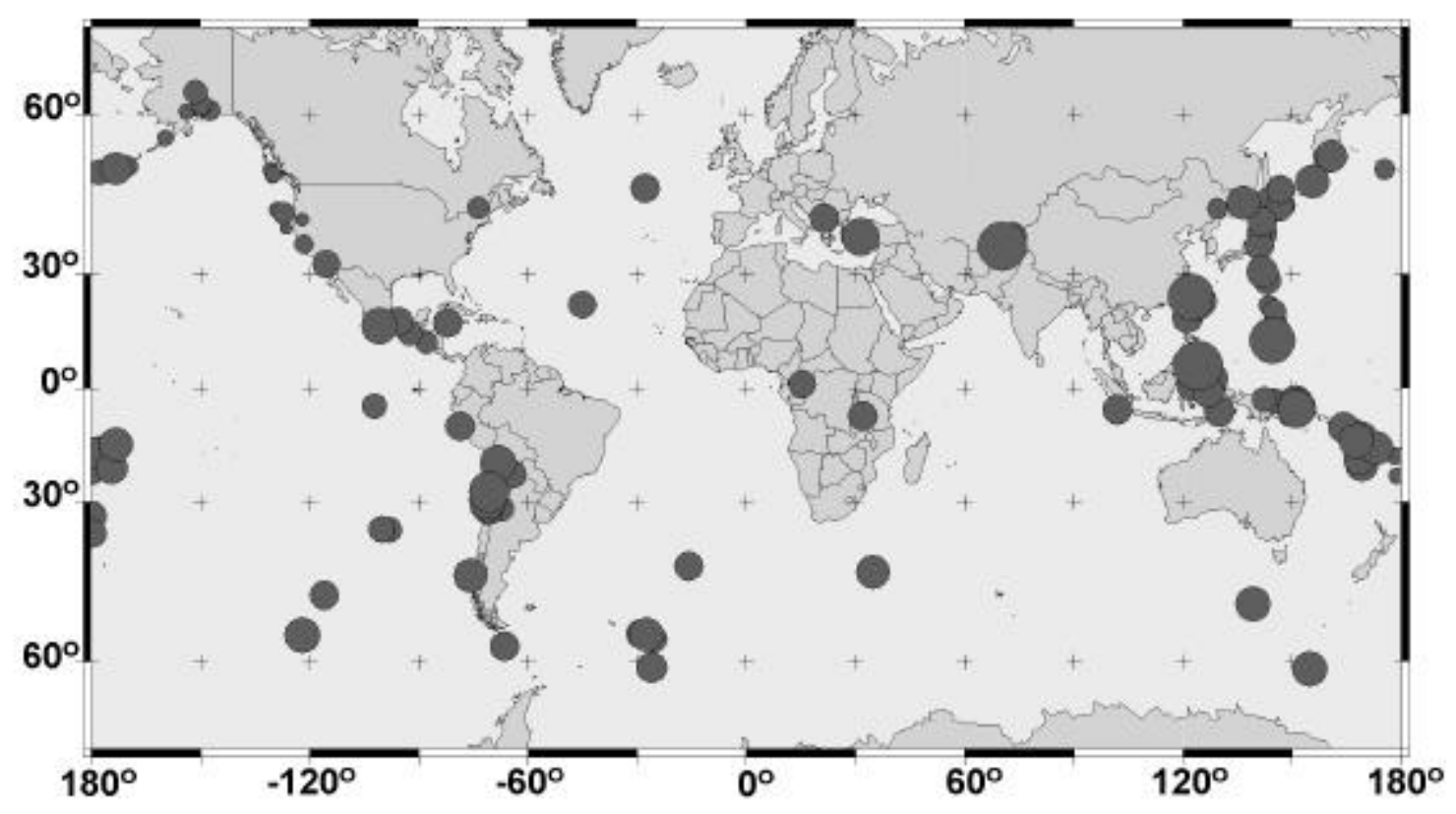

Figure 6: Map showing the locations of teleseisms recorded on the Seattle SHIPS array, with the size of the dot proportional to the earthquake magnitude.

Figs 7-14: Plots of local earthquakes. Data have a 1-14 Hz bandpass filter. Data are arranged by increasing source-receiver distance, with vertical traces on the left, northsouth traces in the center, and east-west traces on the right. The number beneath each figure is the origin time of the event, listed as year.day.hour.minute.second. 


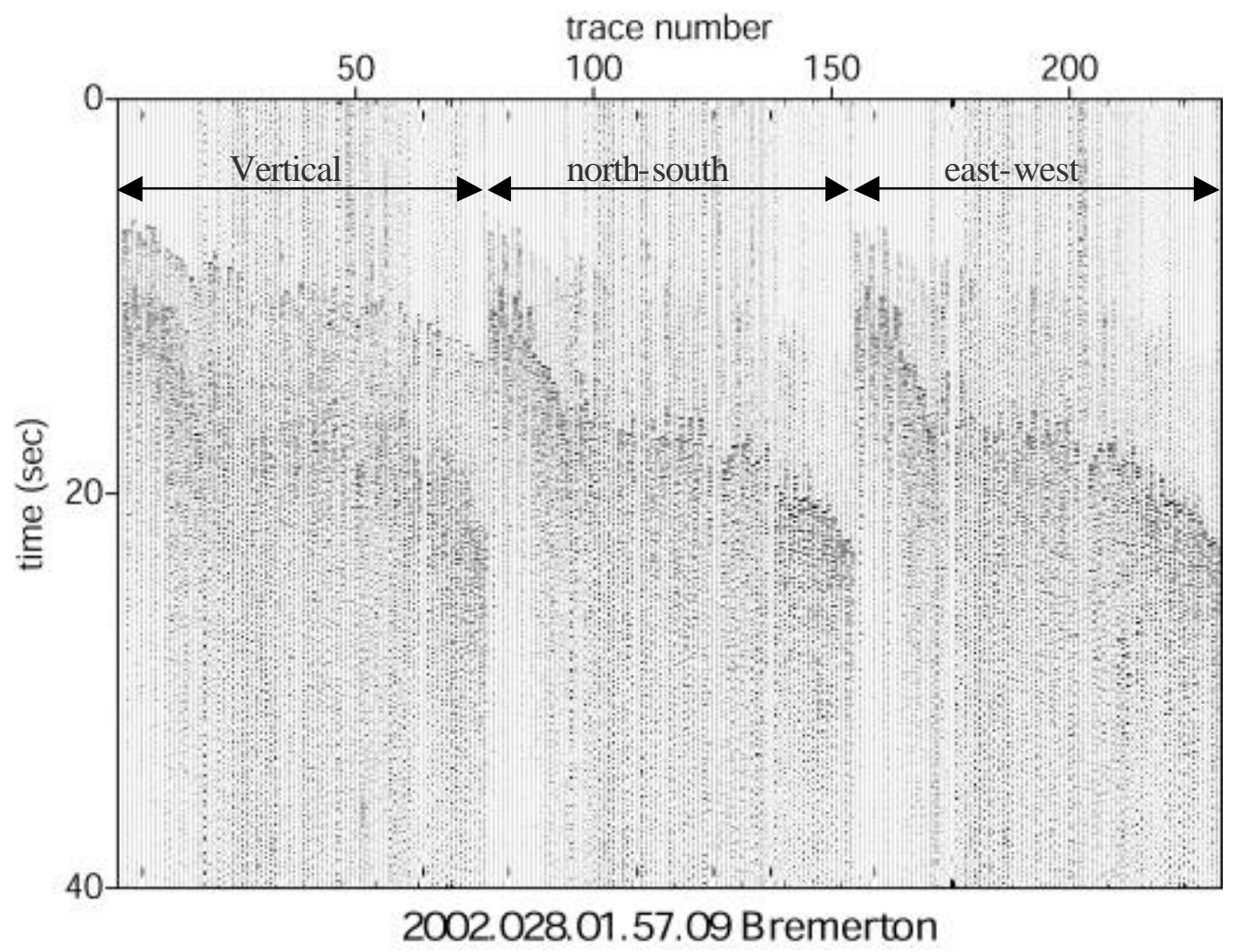

Figure 7a: M2.1 earthquake beneath Bremerton, 19 km deep.

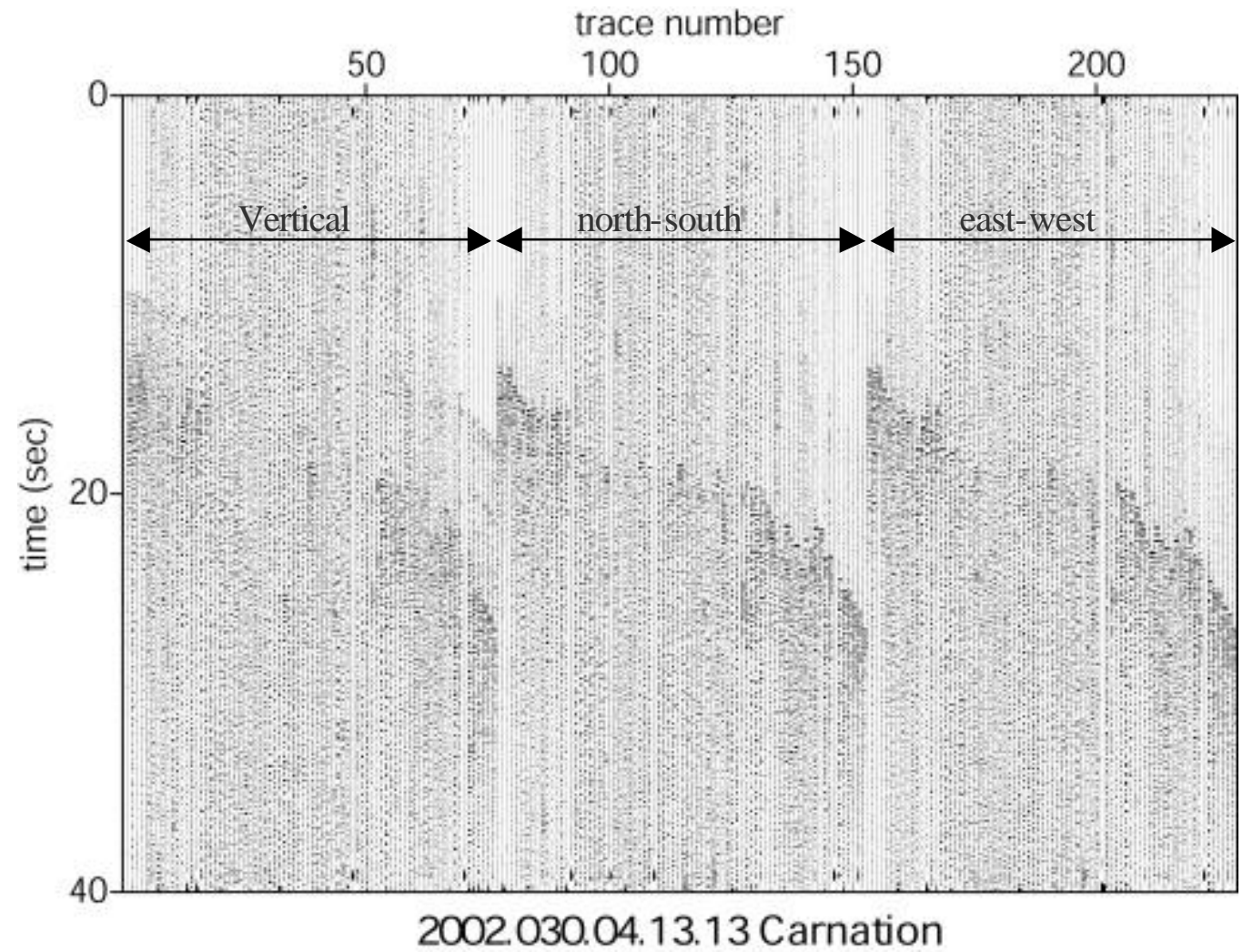

Figure 7b: M2.1 earthquake beneath Carnation, $26 \mathrm{~km}$ deep. 


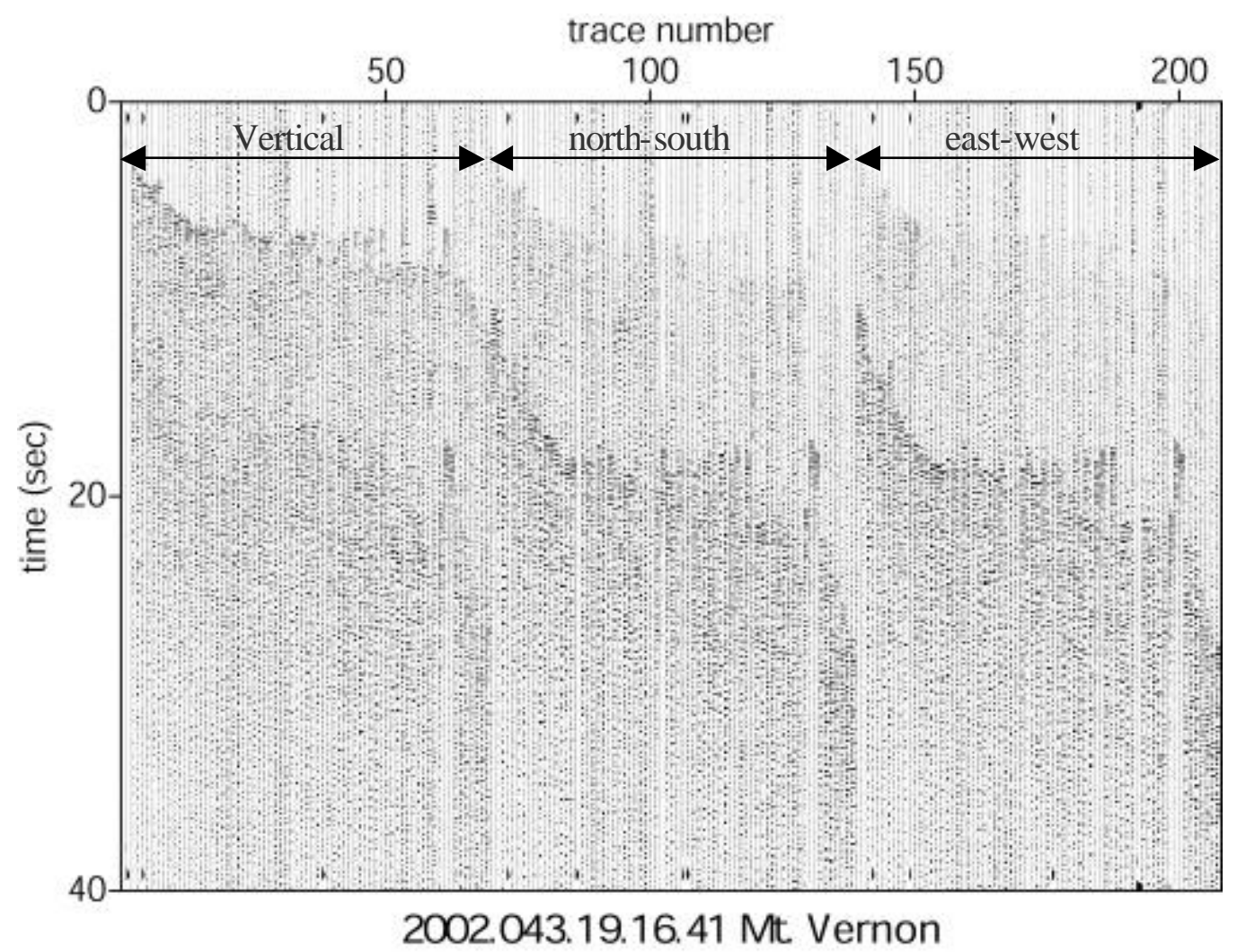

Figure 8a: M3.0 earthquake beneath Mt. Vernon, 19 km deep.

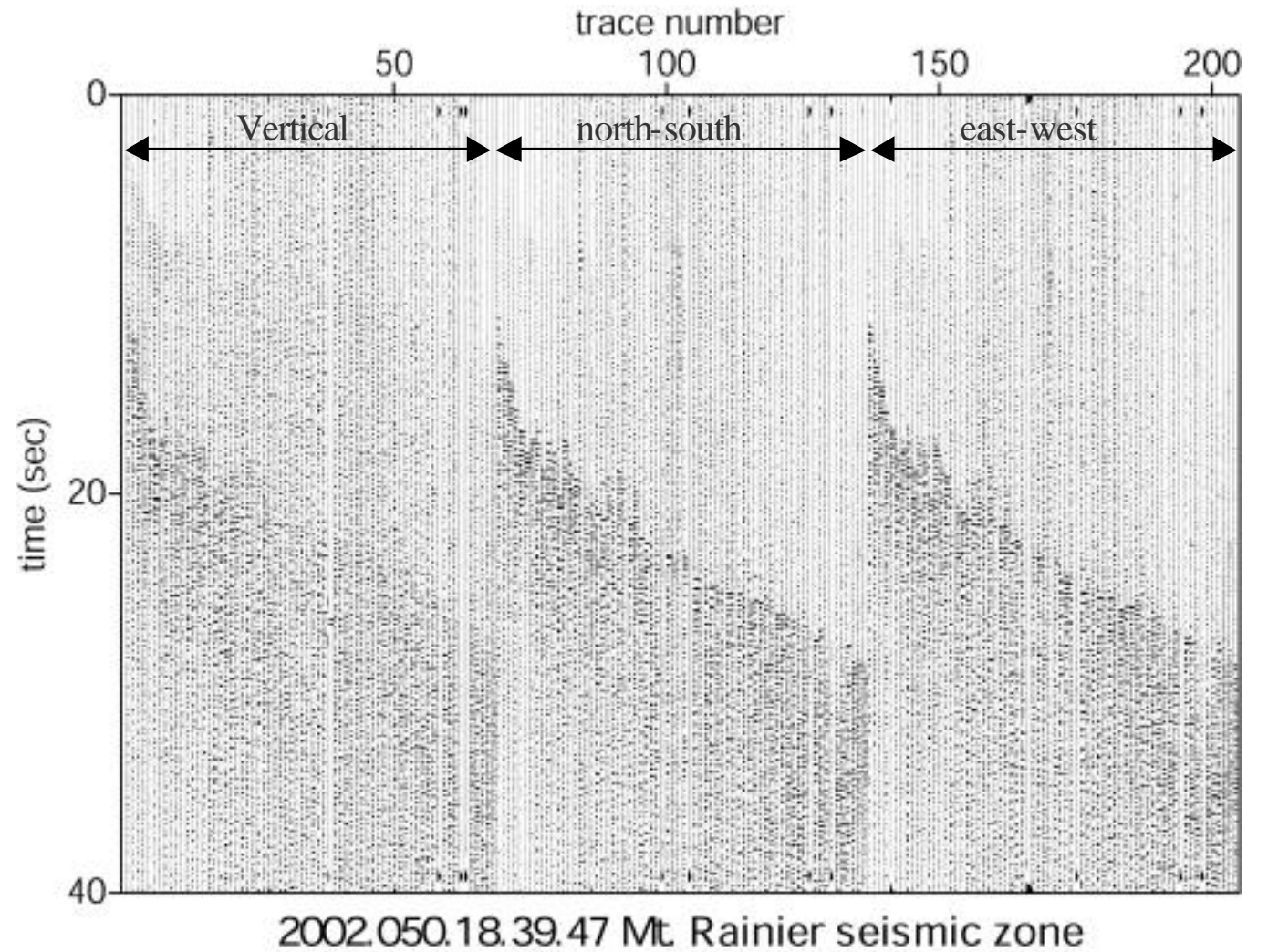

Figure 8b: M3.2 earthquake beneath Mt. Rainier, 0 km deep. 


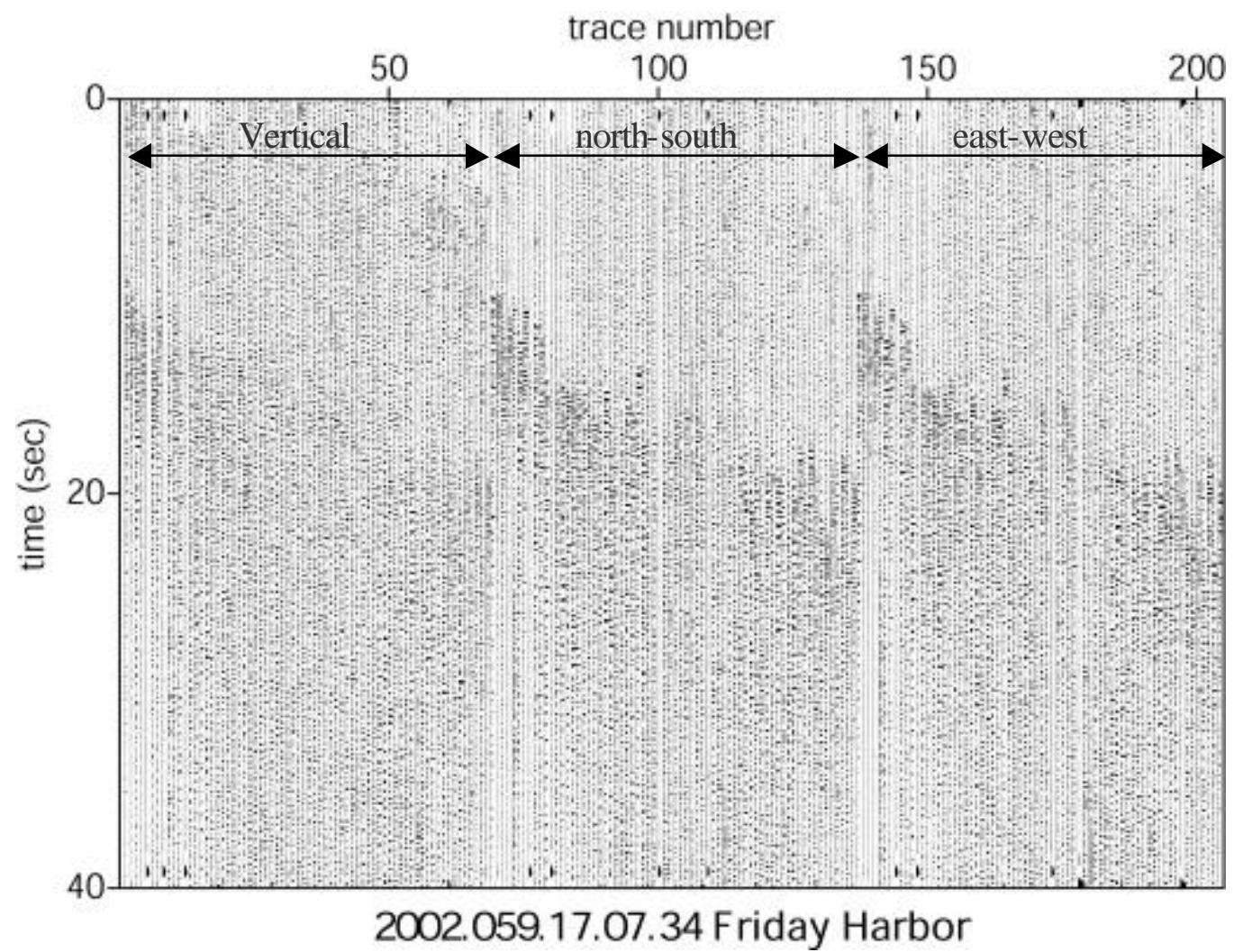

Figure 9a: M2.3 earthquake beneath Friday Harbor, 49 km depth.

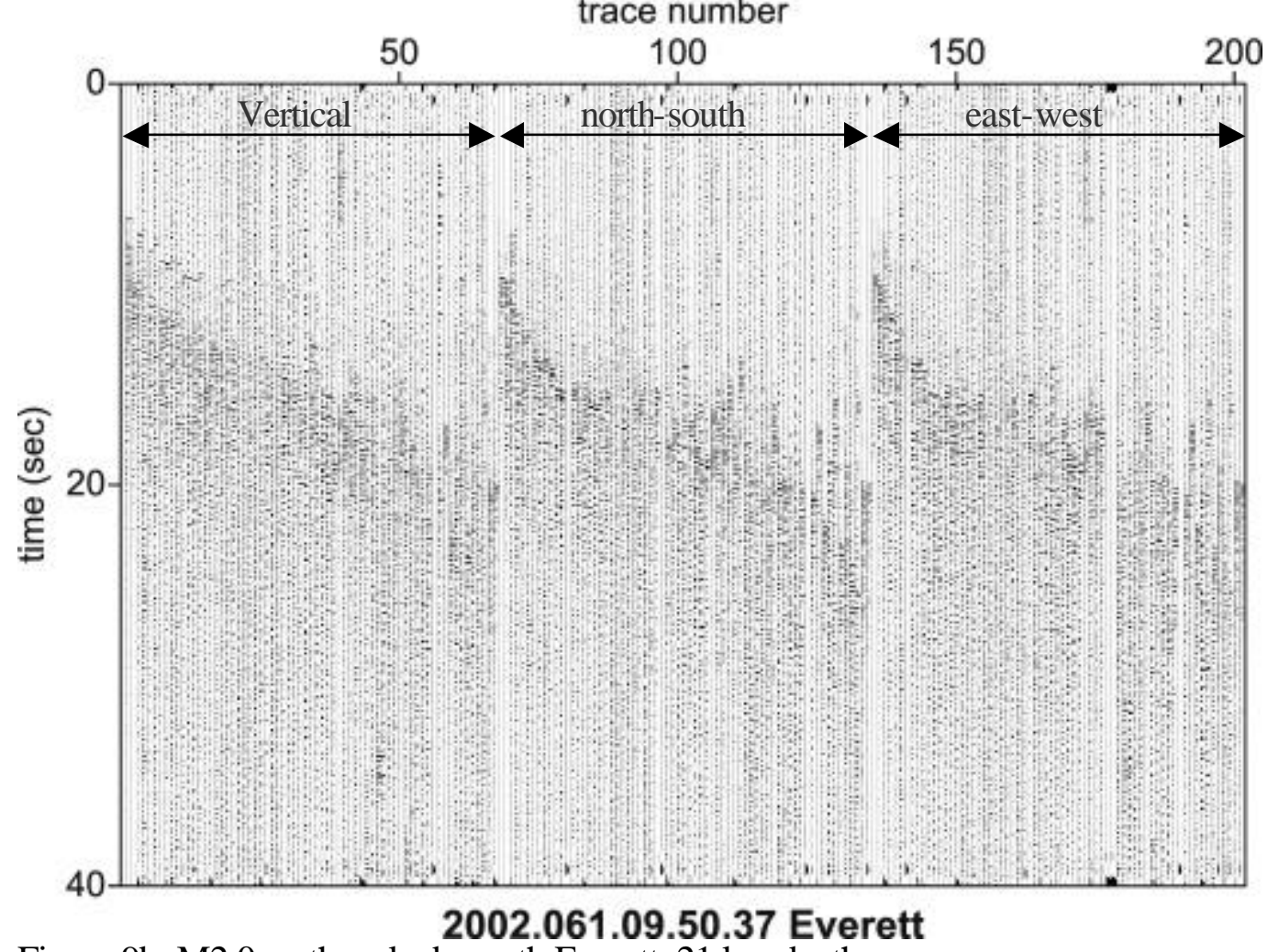

Figure 9b: M2.0 earthquake beneath Everett, $21 \mathrm{~km}$ depth. 


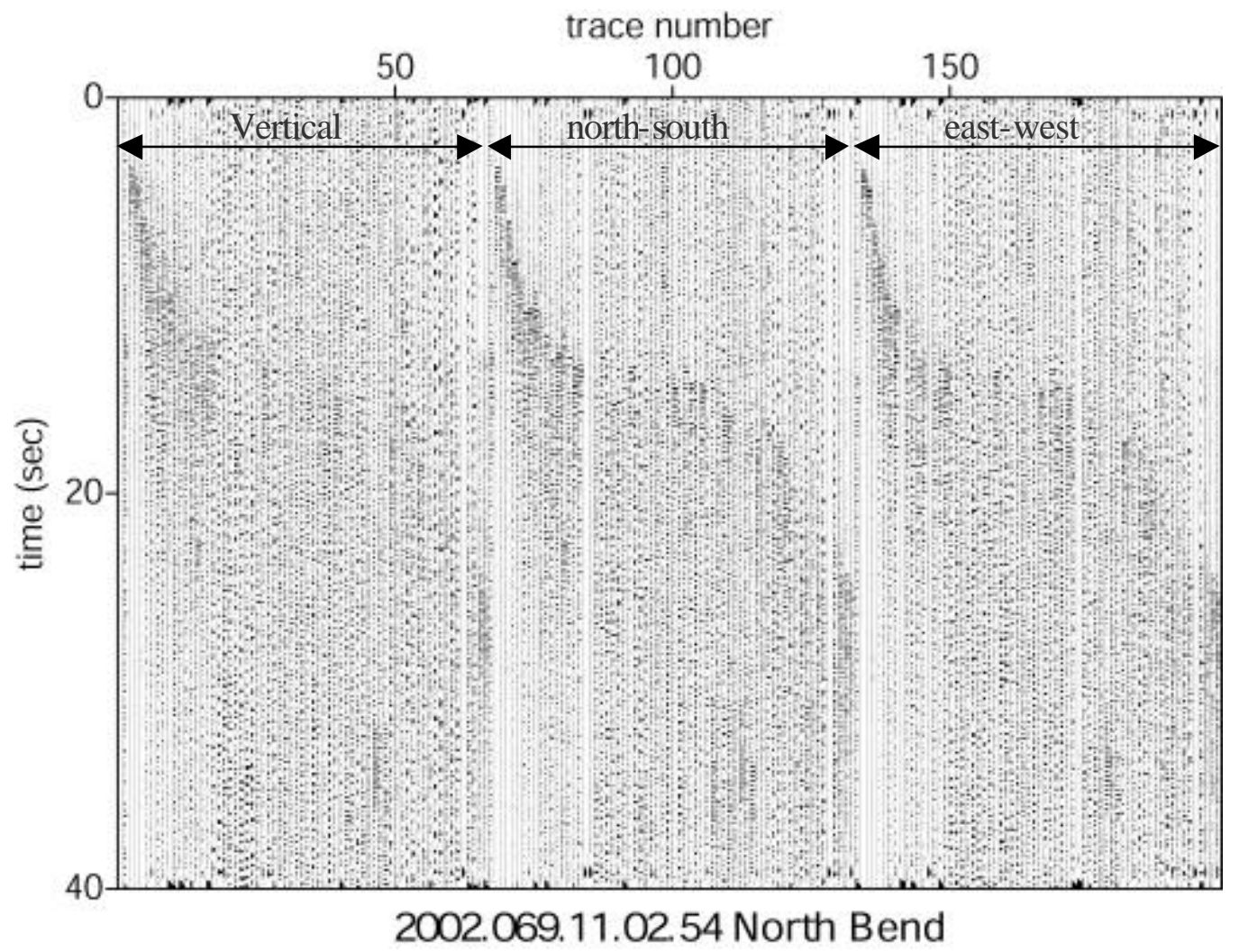

Figure 10a: M1.9 earthquake beneath North Bend, 15 km deep.

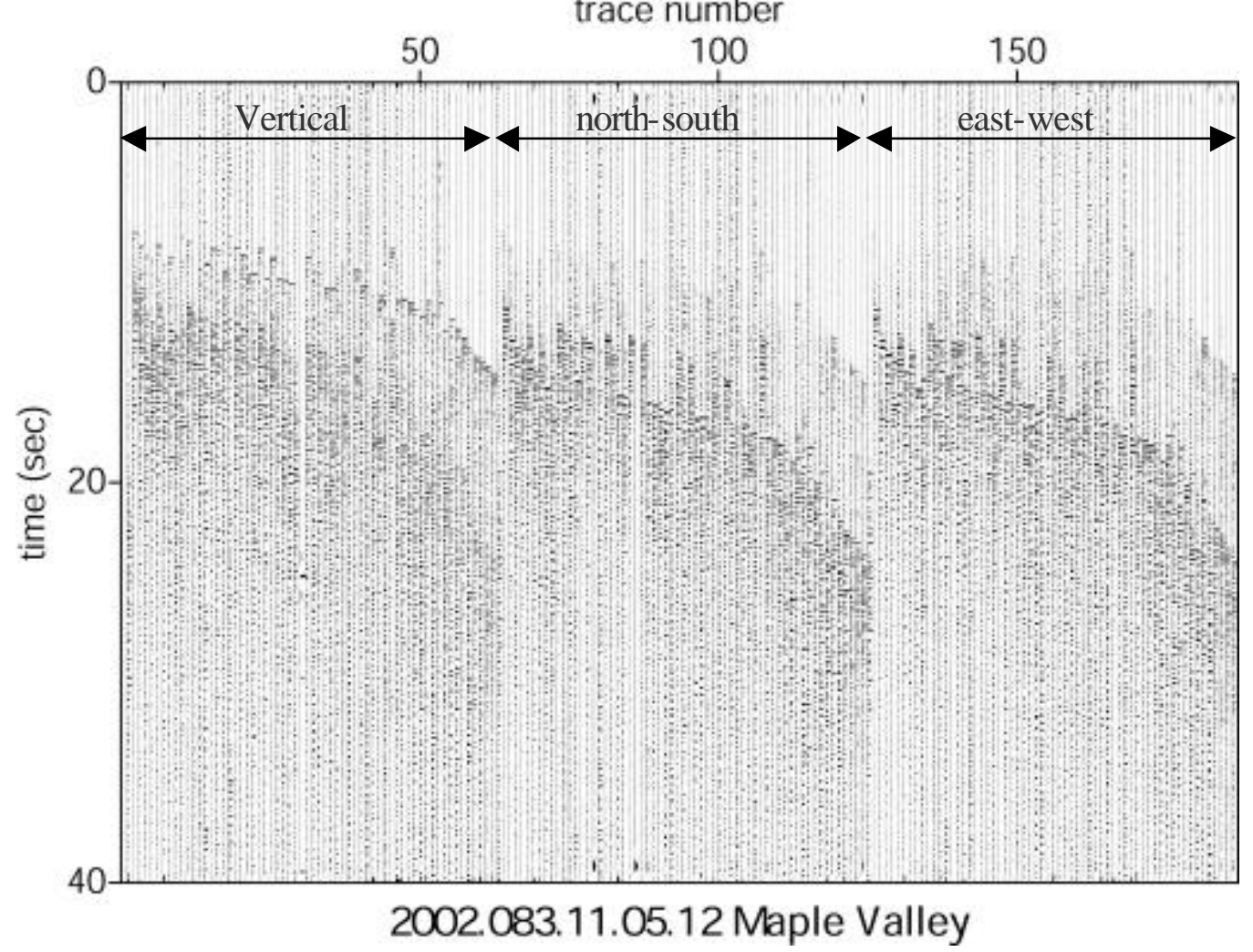

Figure 10b: M2.3 beneath Maple Valley, $20 \mathrm{~km}$ deep. 


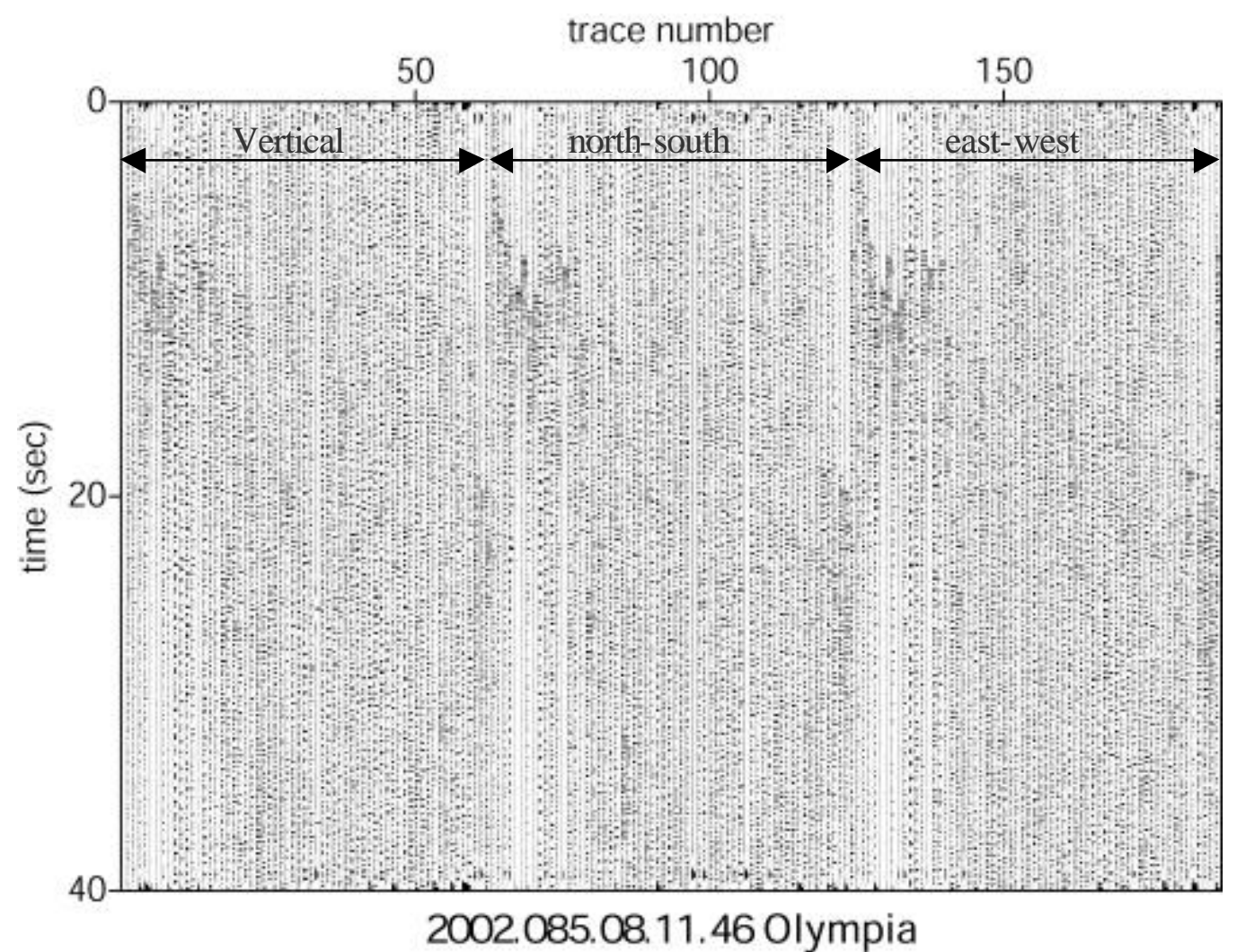

Figure 11a: M1.9 earthquake beneath Olympia, $9 \mathrm{~km}$ deep.



Figure 11b: M2.5 earthquake beneath Tacoma, $18 \mathrm{~km}$ deep. 


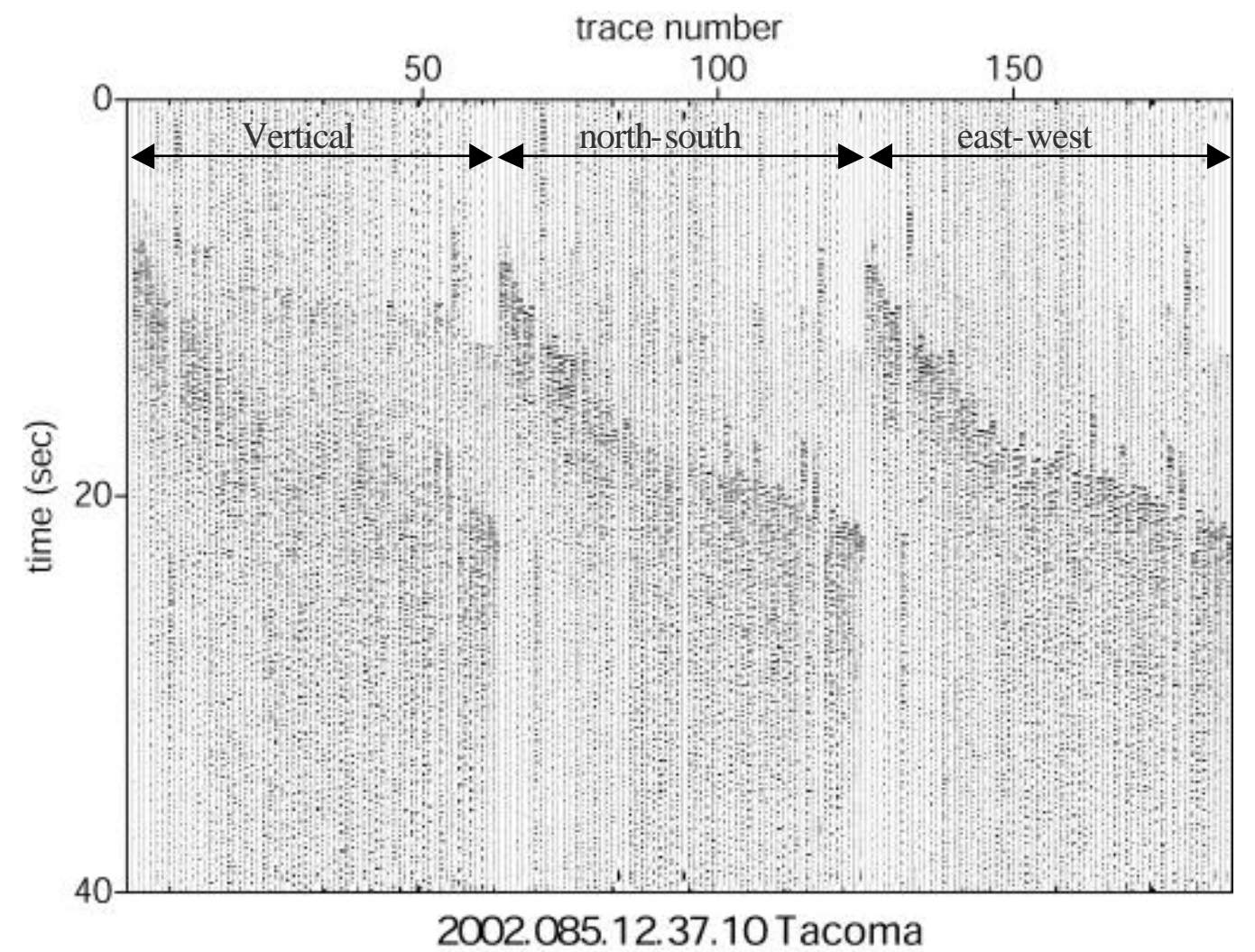

Figure 12a: M2.1 earthquake beneath Tacoma, $18 \mathrm{~km}$ deep.

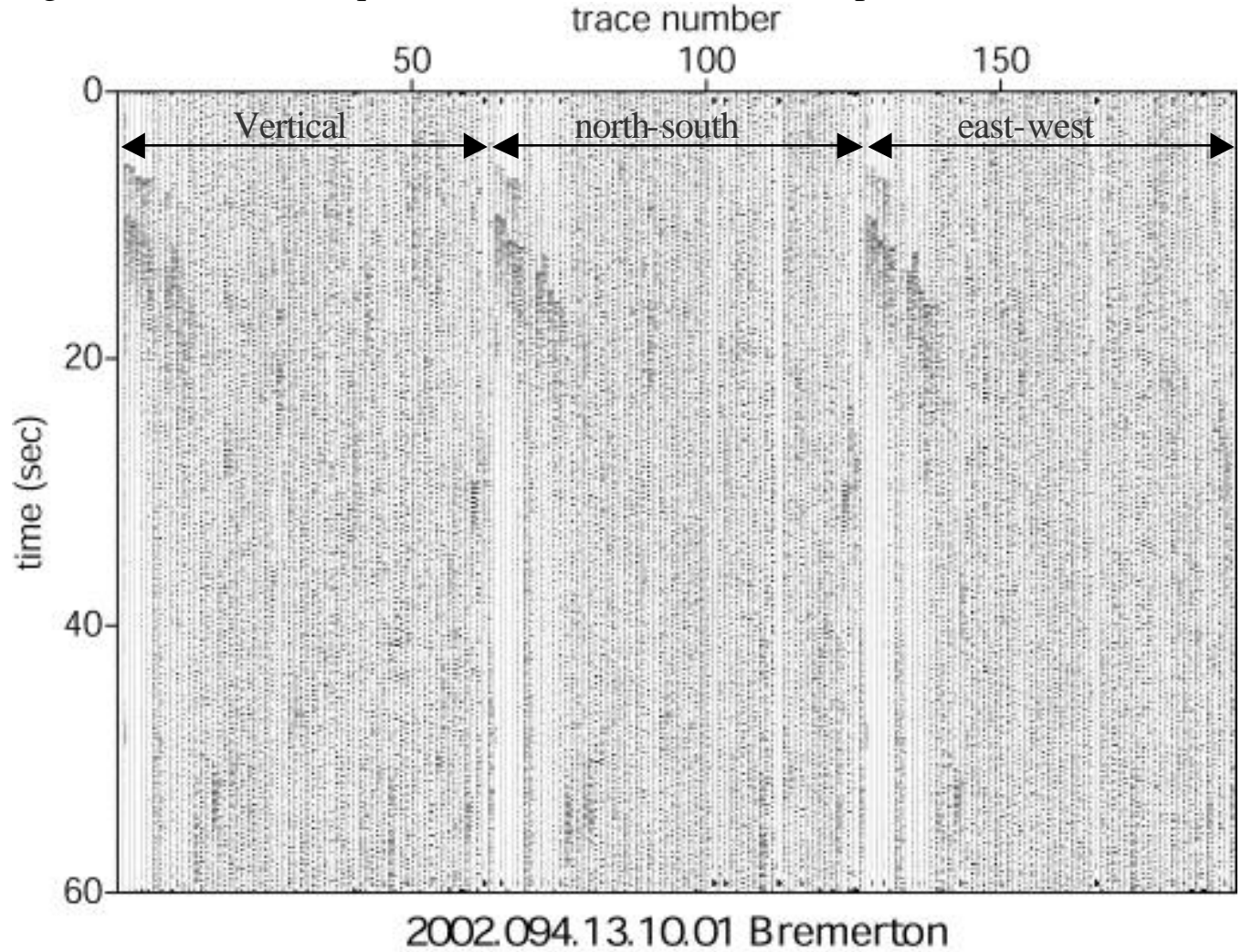

Figure 12b: M1.8 earthquake beneath Bremerton, $20 \mathrm{~km}$ deep. 


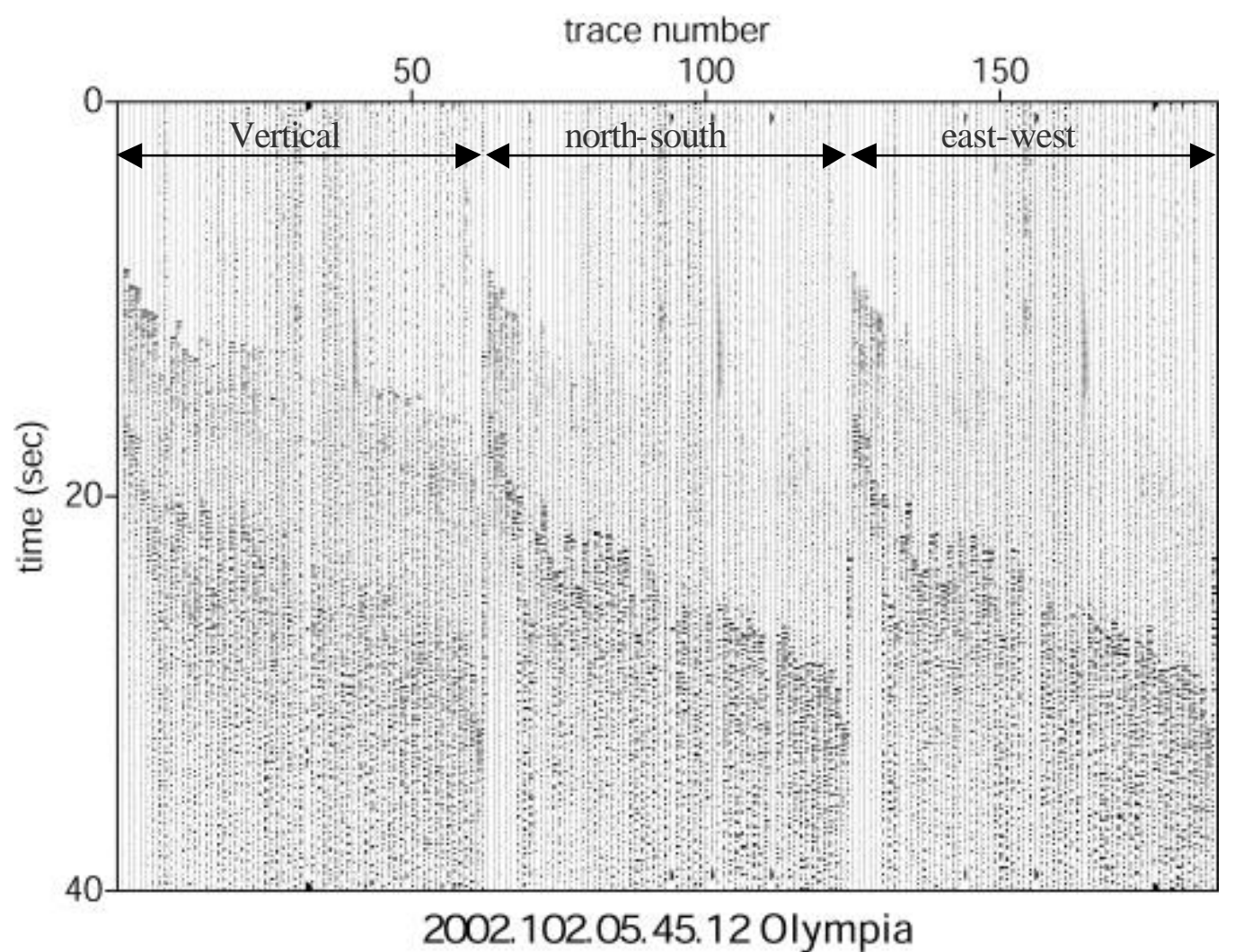

Figure 13a: M2.7 earthquake beneath Olympia, $43 \mathrm{~km}$ deep.

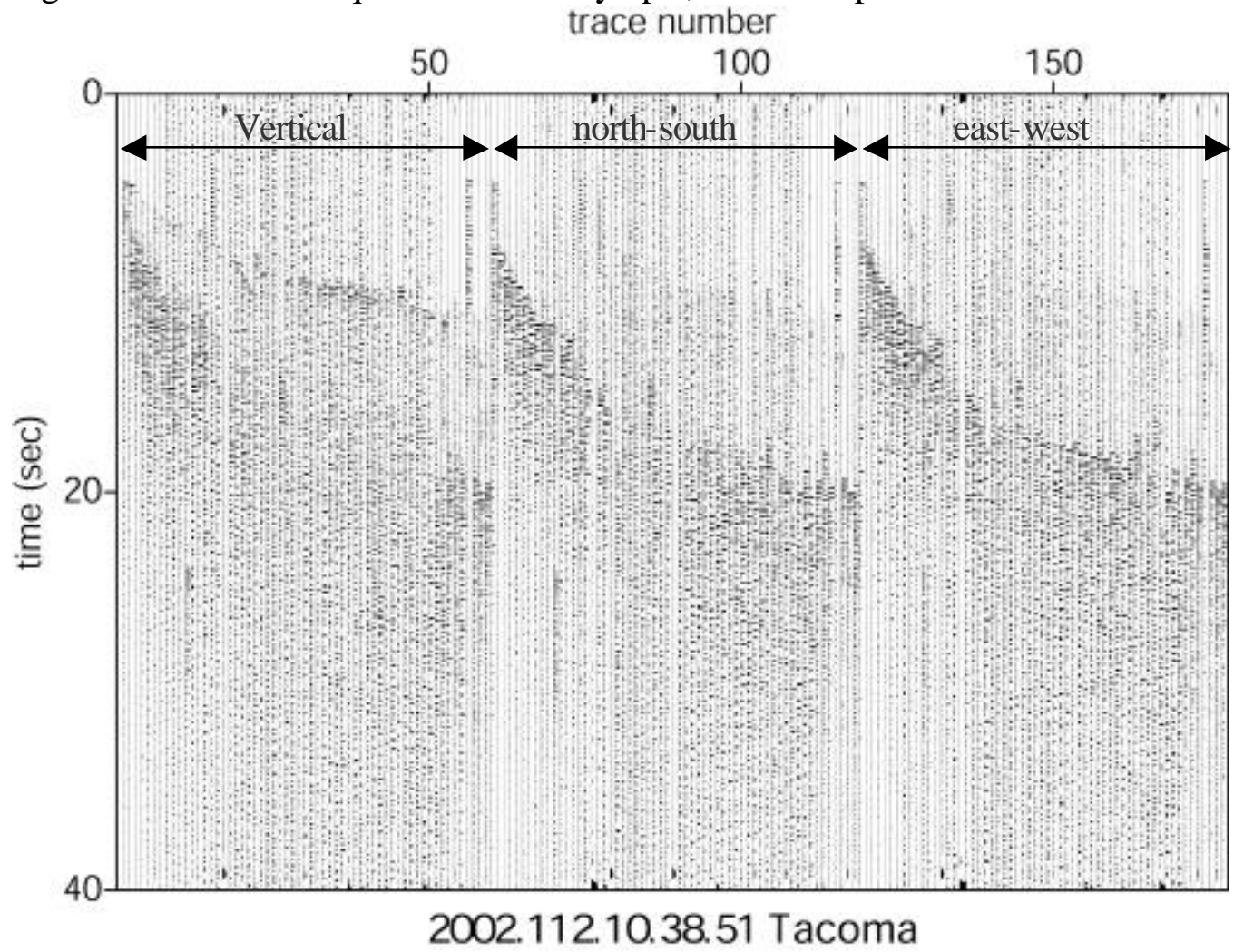

Figure 13b: M2.2 earthquake beneath Tacoma, $20 \mathrm{~km}$ deep. 


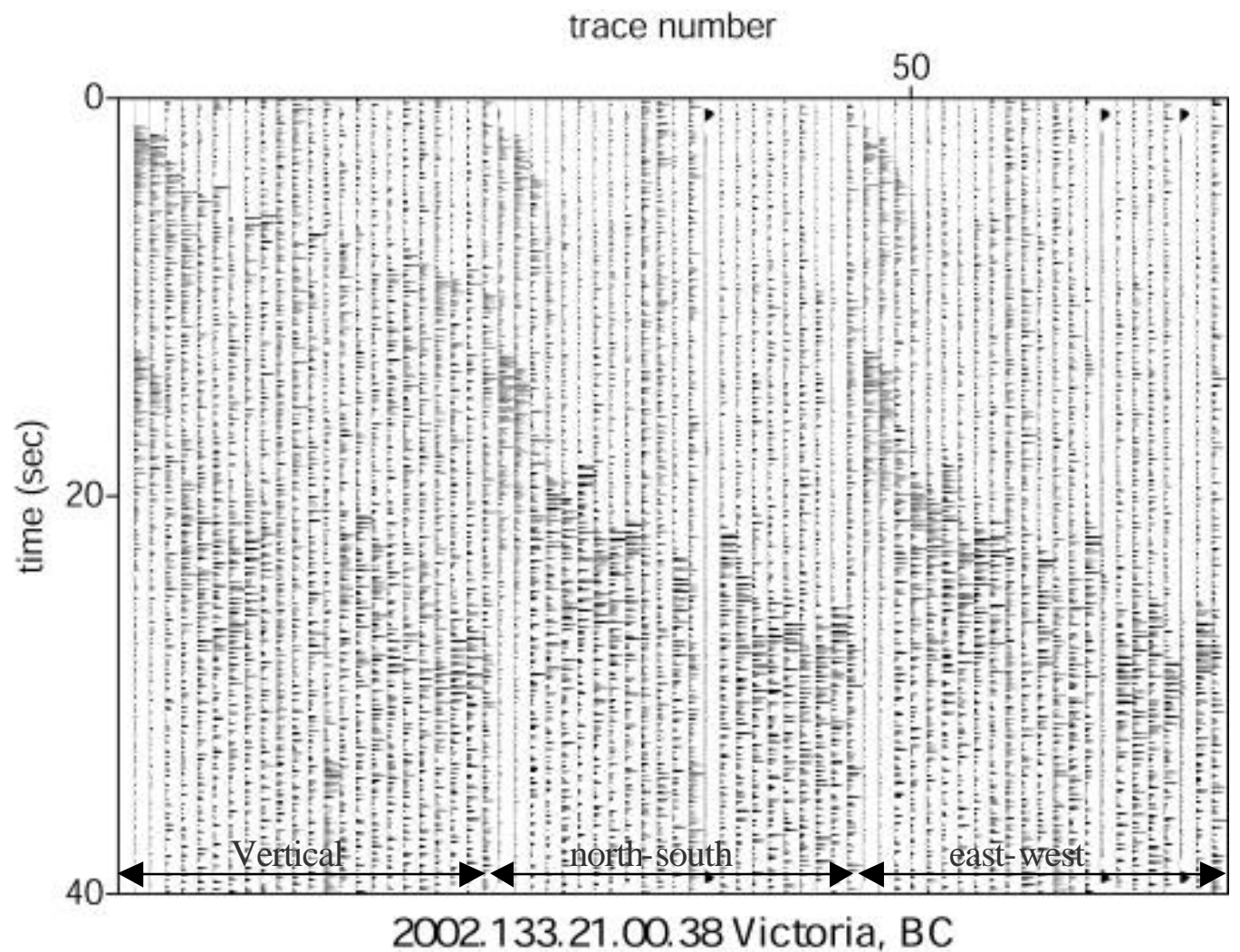

Figure 14a: M2.7 earthquake beneath Victoria, Canada, $43 \mathrm{~km}$ deep. Only 23 stations remaining. trace number

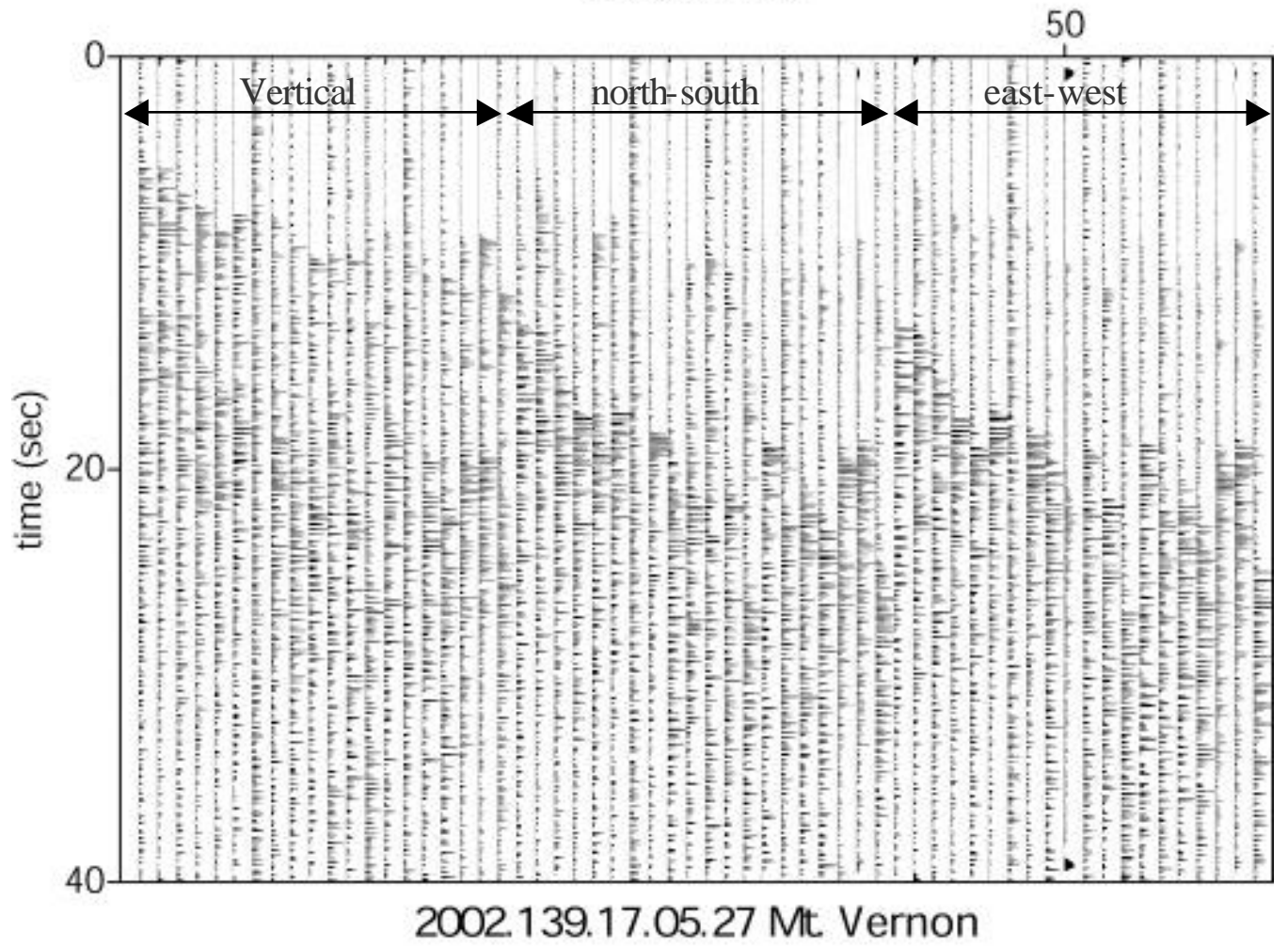

Figure 14b: M2.7 earthquake beneath Mt. Vernon, $14 \mathrm{~km}$ deep. Only 20 stations remaining. 


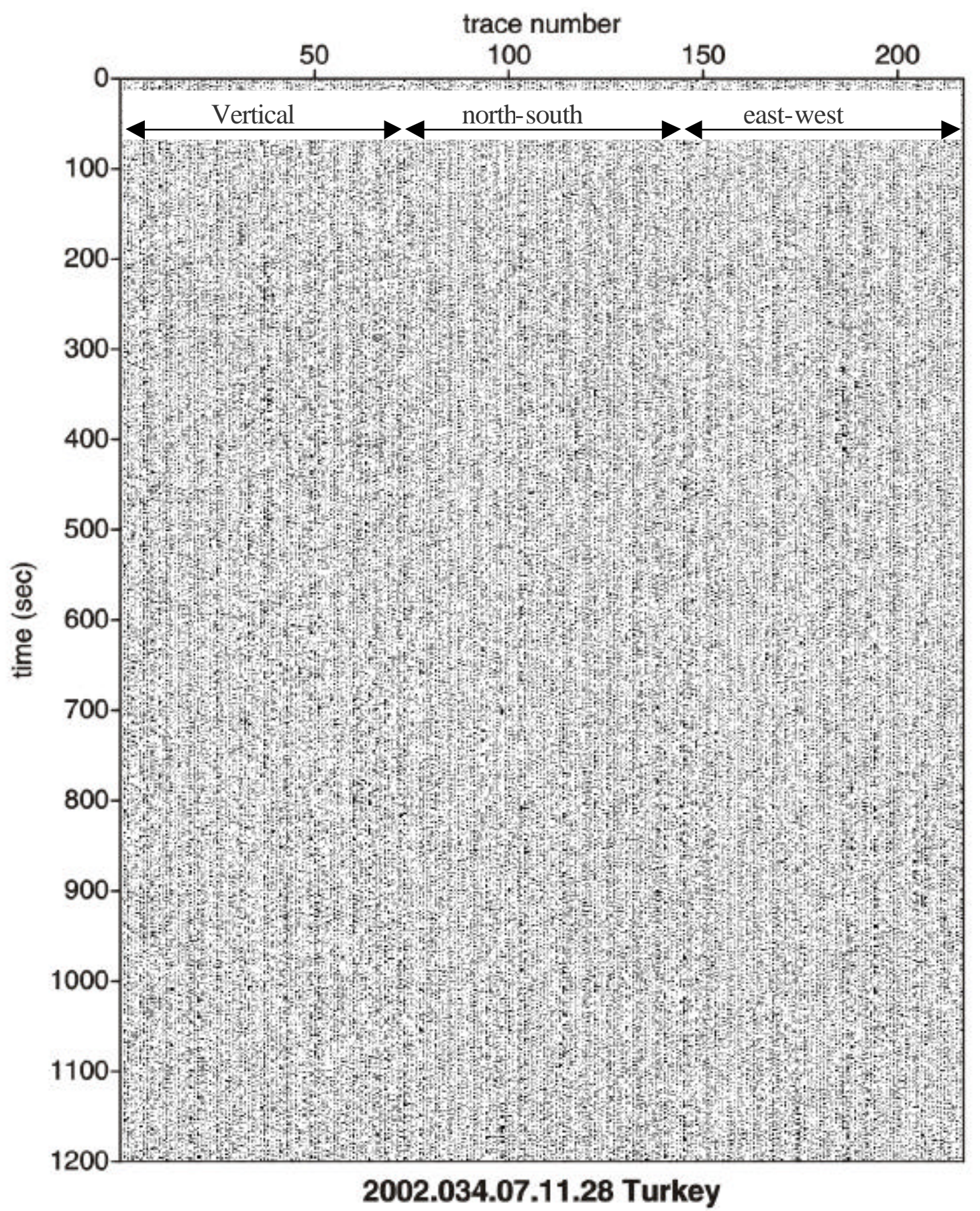

Figure 15: M6.5 earthquake in Turkey recorded on the SHIPS seismic array, with the vertical component data on the left third of the plot, the north-south component data in the middle third, and the east-west component data on the right third. The earthquake occurred at 7:11 GMT (23:11 local time) and has an epicentral distance of about $91^{\circ}$. Note that little signal is recorded, as was the case for most of the teleseisms with magnitudes of 6.5 or less. The data have a $0.05,0.1,0.8,1.4 \mathrm{~Hz}$ trapezoidal bandpass filter. 




2002.062.12.08.12 Afghanistan (2 events 10 secs apart)

Figure 16: M7.4 earthquake in Afghanistan recorded on the SHIPS seismic array, with the vertical component data on the left third of the plot, the north-south component data in the middle third, and the east-west component data on the right third. The earthquake occurred at 12:08 GMT (4:08 local time) and has an epicentral distance of about $95^{\circ}$. There is a high signal-to-noise ratio because of low noise levels at 4 a.m. local time. The event was at a depth of about $250 \mathrm{~km}$, and the P, sP, PP, pPP, SkS, S and PS phases (listed in order of arrival time) are visible. The data have a $0.05,0.1,0.8,1.4 \mathrm{~Hz}$ trapezoidal bandpass filter. 


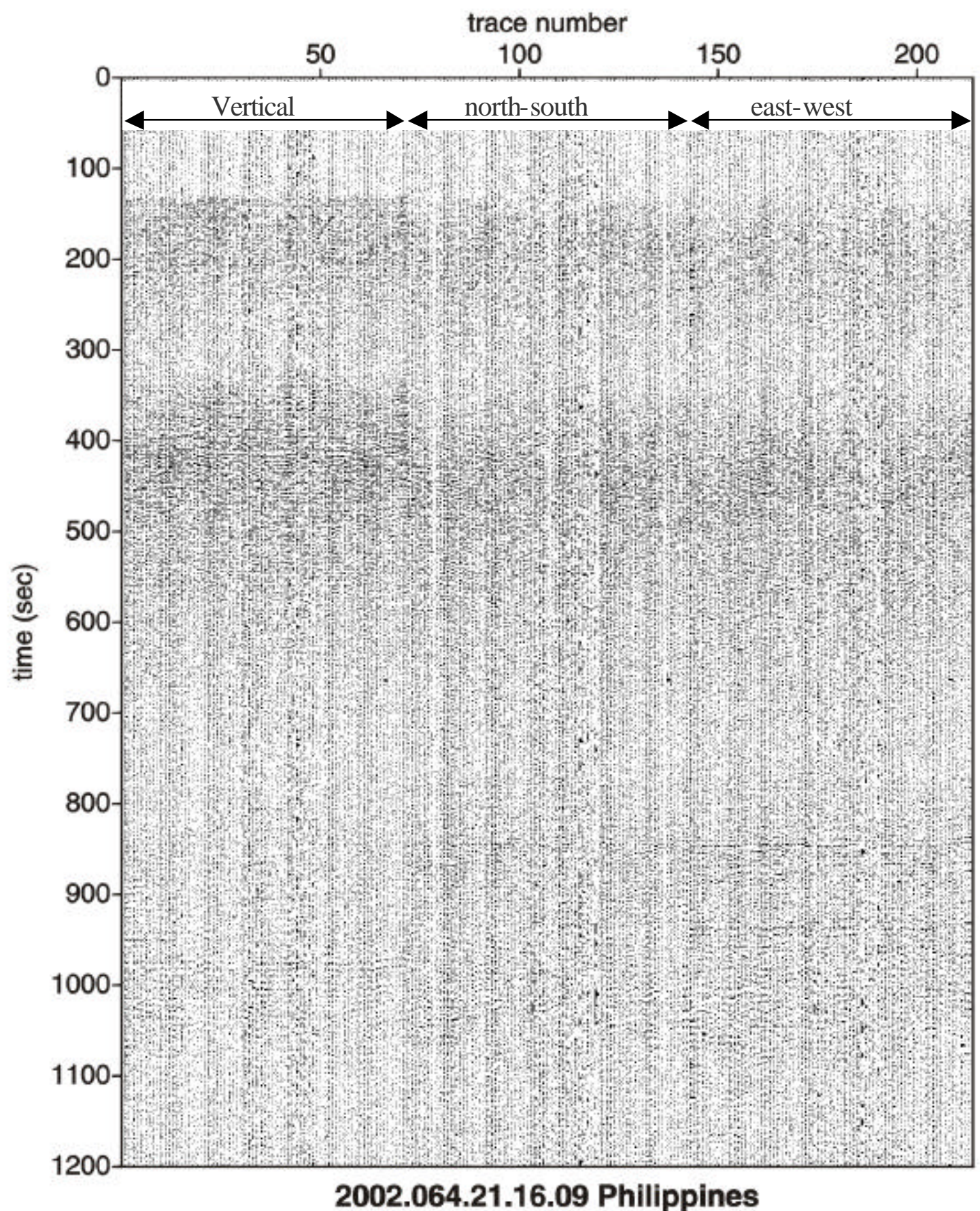

Figure 17: M7.5 earthquake in he Philippines recorded on the SHIPS seismic array, with the vertical component data on the left third of the plot, the north-south component data in the middle third, and the east-west component data on the right third. The earthquake occurred at 21:16 GMT (13:21 local time) and has an epicentral distance of about $101^{\circ}$. The $\mathrm{P}$ and $\mathrm{S}$ waves arrivals are obvious, although the signal-to-noise ratio is only moderately good because of high noise levels in the early afternoon. The data have a $0.05,0.1,0.8,1.4 \mathrm{~Hz}$ trapezoidal bandpass filter. 


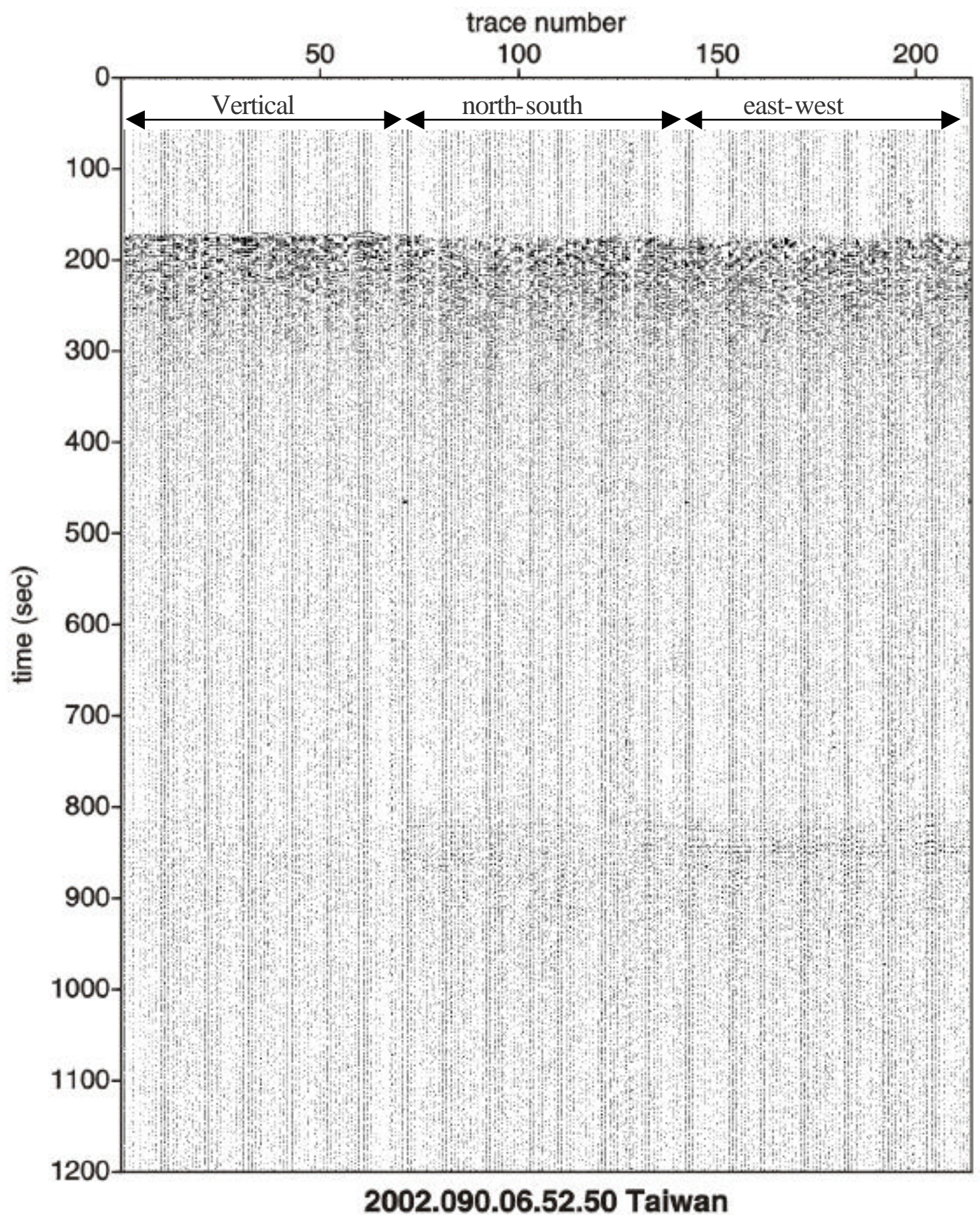

Figure 18: M7.1 earthquake in Taiwan recorded on the SHIPS seismic array, with the vertical component data on the left third of the plot, the north-south component data in the middle third, and the east-west component data on the right third. The earthquake occurred at 6:52 GMT (22:52 local time) and has an epicentral distance of about $88^{\circ}$. The $\mathrm{P}$ and $\mathrm{S}$ waves arrivals are obvious and have a good signal-to-noise ratio. The data have a $0.05,0.1,0.8,1.4 \mathrm{~Hz}$ trapezoidal bandpass filter. 


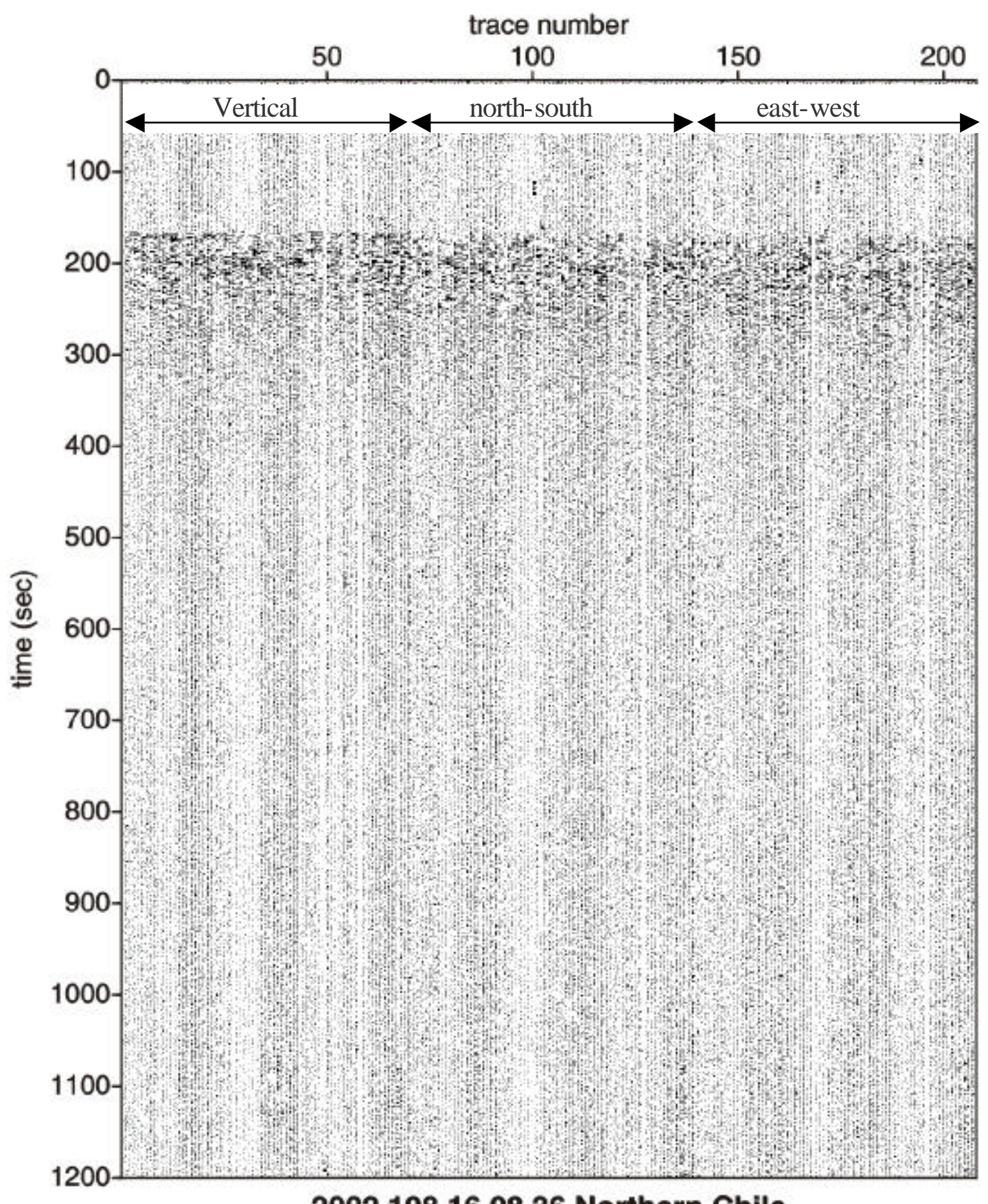

\subsection{Northern Chile}

Figure 19: M6.7 earthquake in northern Chile recorded on the SHIPS seismic array, with the vertical component data on the left third of the plot, the north-south component data in the middle third, and the east-west component data on the right third. The earthquake occurred at 16:08 GMT (8:08 local time) and has an epicentral distance of about $88^{\circ}$. The $\mathrm{P}$ wave arrivals are obvious and have a good signal-to-noise ratio, but the $\mathrm{S}$-wave arrivals are barely visible at about $800 \mathrm{sec}$ on this plot. The data have a $0.05,0.1,0.8,1.4 \mathrm{~Hz}$ trapezoidal bandpass filter. 


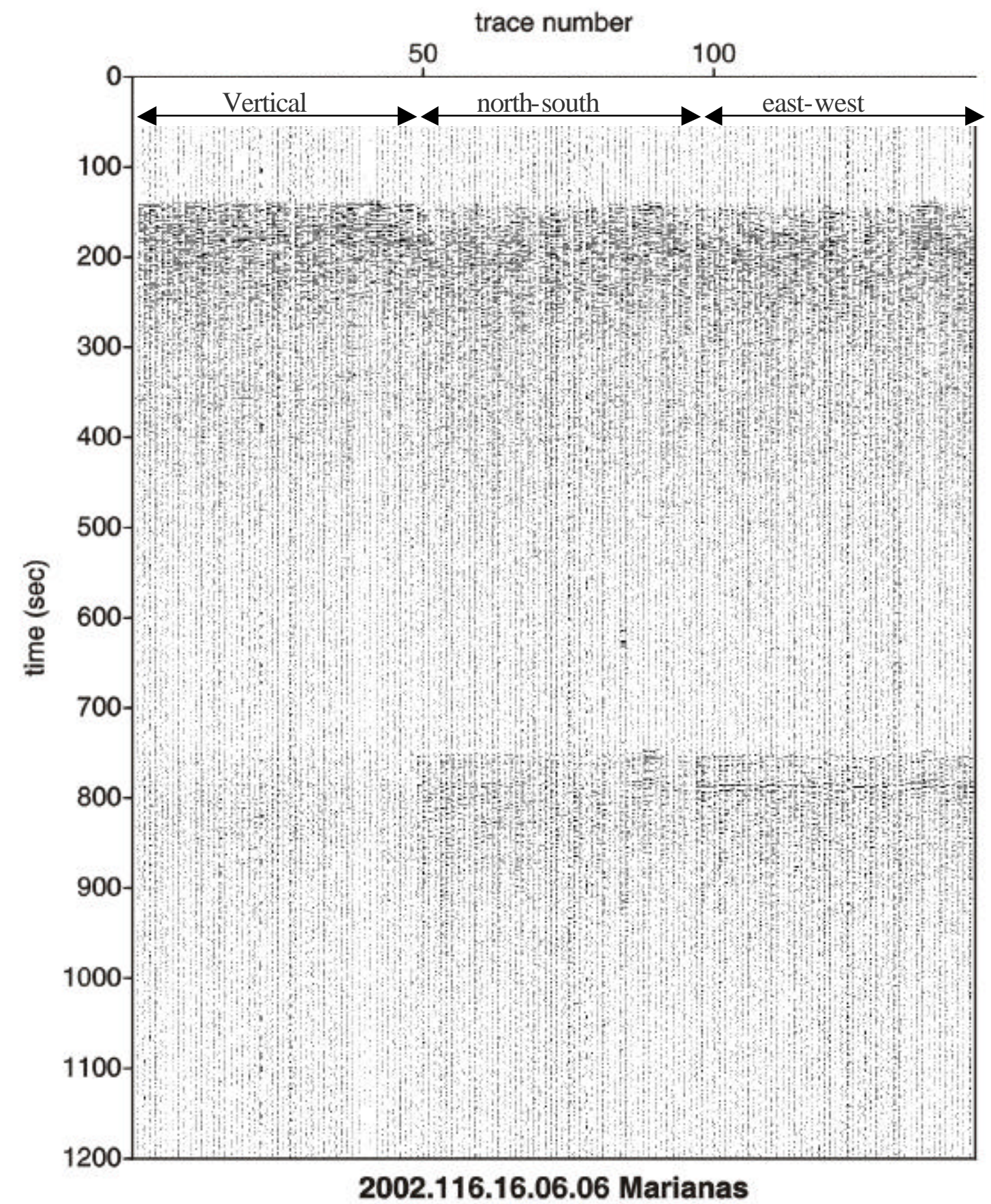

Figure 20: M7.1 earthquake in the Marianas recorded on the SHIPS seismic array, with the vertical component data on the left third of the plot, the north-south component data in the middle third, and the east-west component data on the right third. The earthquake occurred at 16:06 GMT (8:06 local time) and has an epicentral distance of about $82^{\circ}$. The $\mathrm{P}$ and $\mathrm{S}$ wave arrivals are obvious and have a good signal-to-noise ratio, despite high noise levels at the 8:06 a.m. local time of the arrivals. The data have a 0.05, 0.1, 0.8, 1.4 $\mathrm{Hz}$ trapezoidal bandpass filter. Only about 50 stations were operating at the time of the earthquake, so there are fewer traces on this plot than on previous plots. 


\section{APPENDIX A: SITE VISITS, INSTRUMENT STATUS, AND DOWNLOADS}

(Following pages.) Graph showing the time of station operation, maintenance visits, and instrument status. Rows list each site, instrument (DAS) number, maintenance route (team) number, disk size, and instrument status each day (columns). Colors represent the instrument status, with green meaning everything working, blue meaning the GPS was not functioning, yellow meaning there were sensor problems, red meaning DAS failure (no data), and salmon meaning that no instrument was present at the site. Each maintenance visit is labeled with the number of megabytes of data that were downloaded. The label "in" marks when instruments were installed, "dead/in," "pull/in" and "\#\#\#/in" mark days in which instruments were changed at the site (instrument numbers listed sequentially in $4^{\text {th }}$ column). Significant GPS locks, preceding or following long periods with no locks, are denoted by the "^^" symbols. "Full" denotes days when disks were predicted to fill, but can be ignored because data were downloaded before the disks filled. 


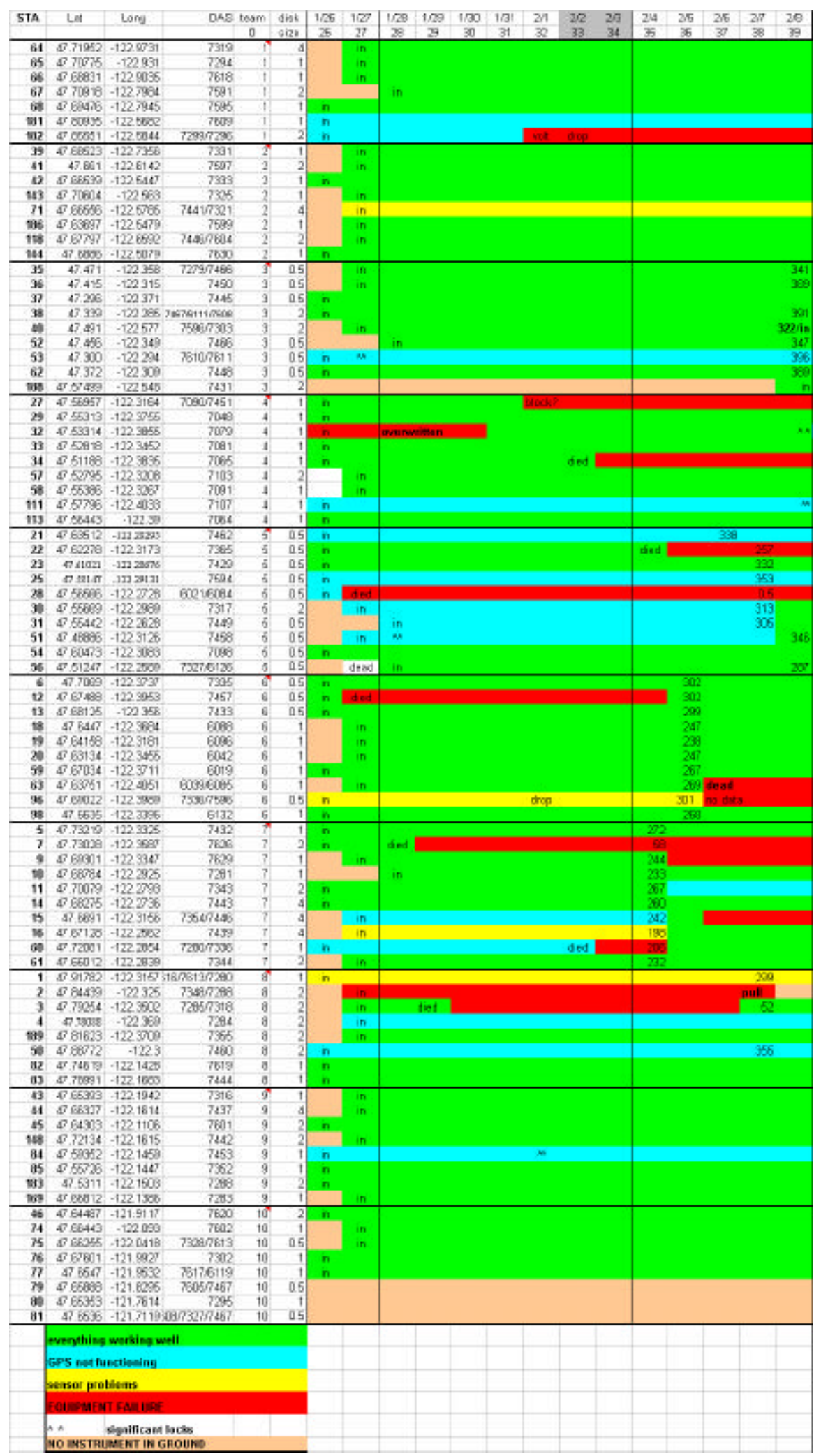




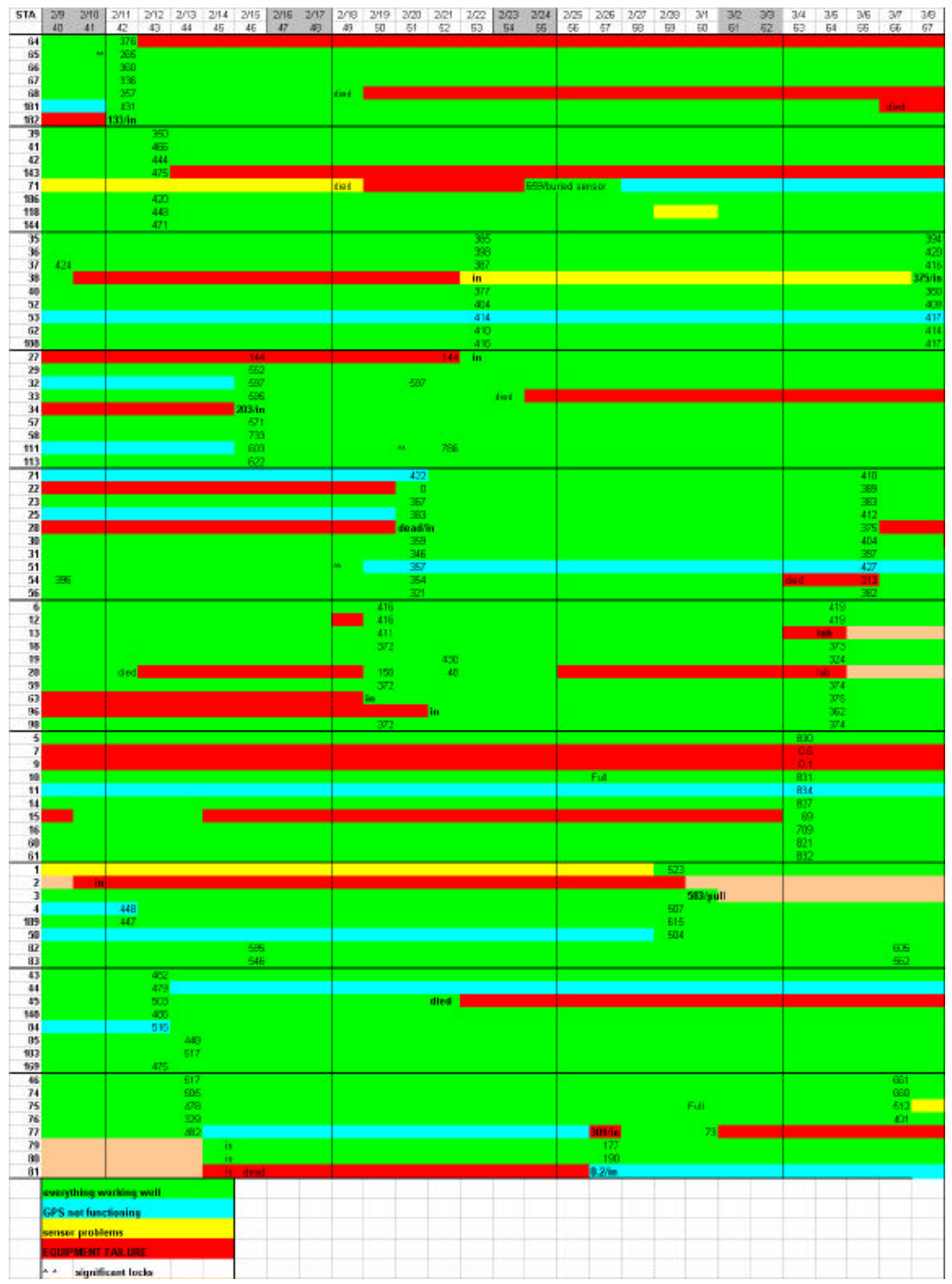




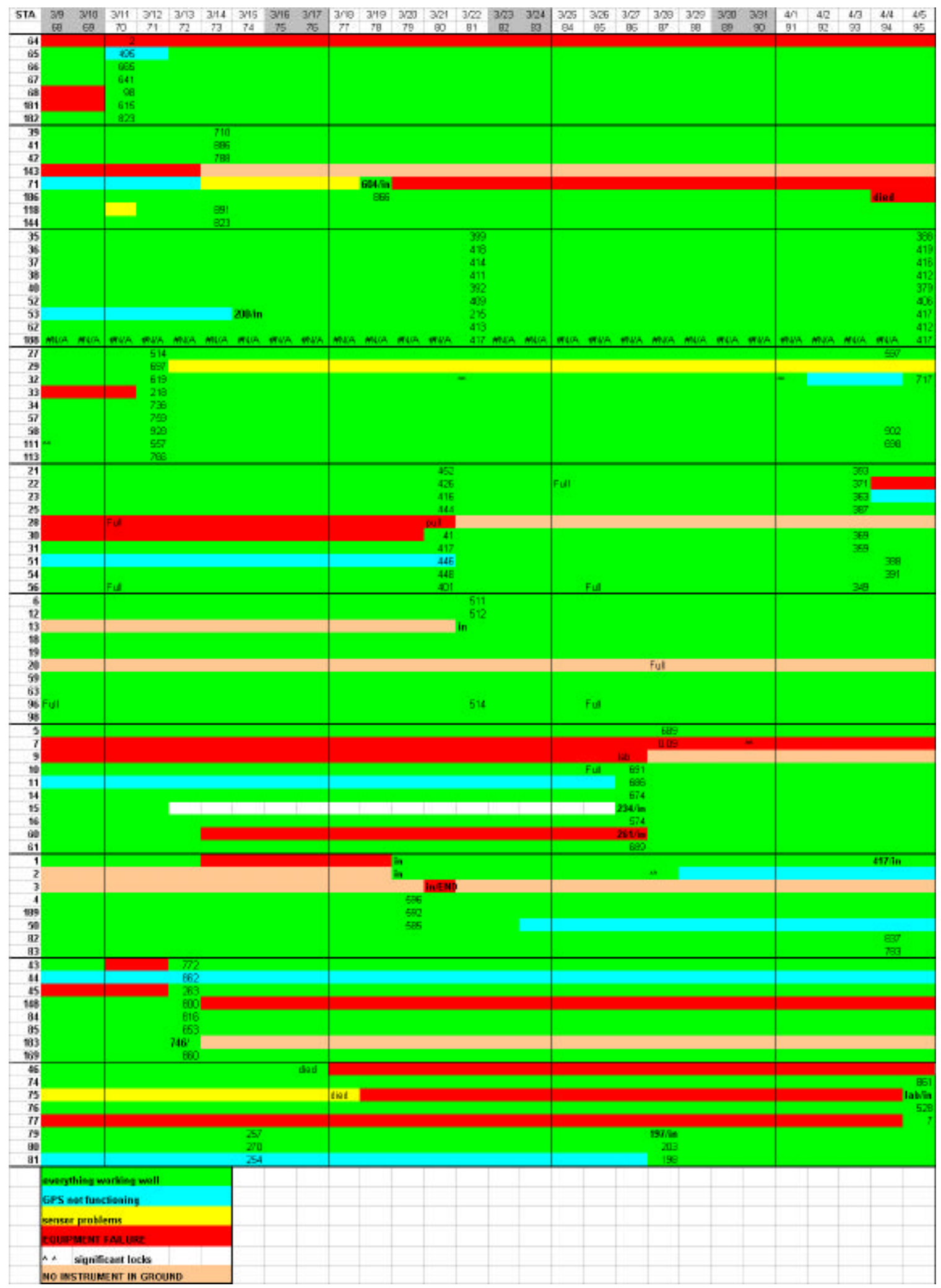




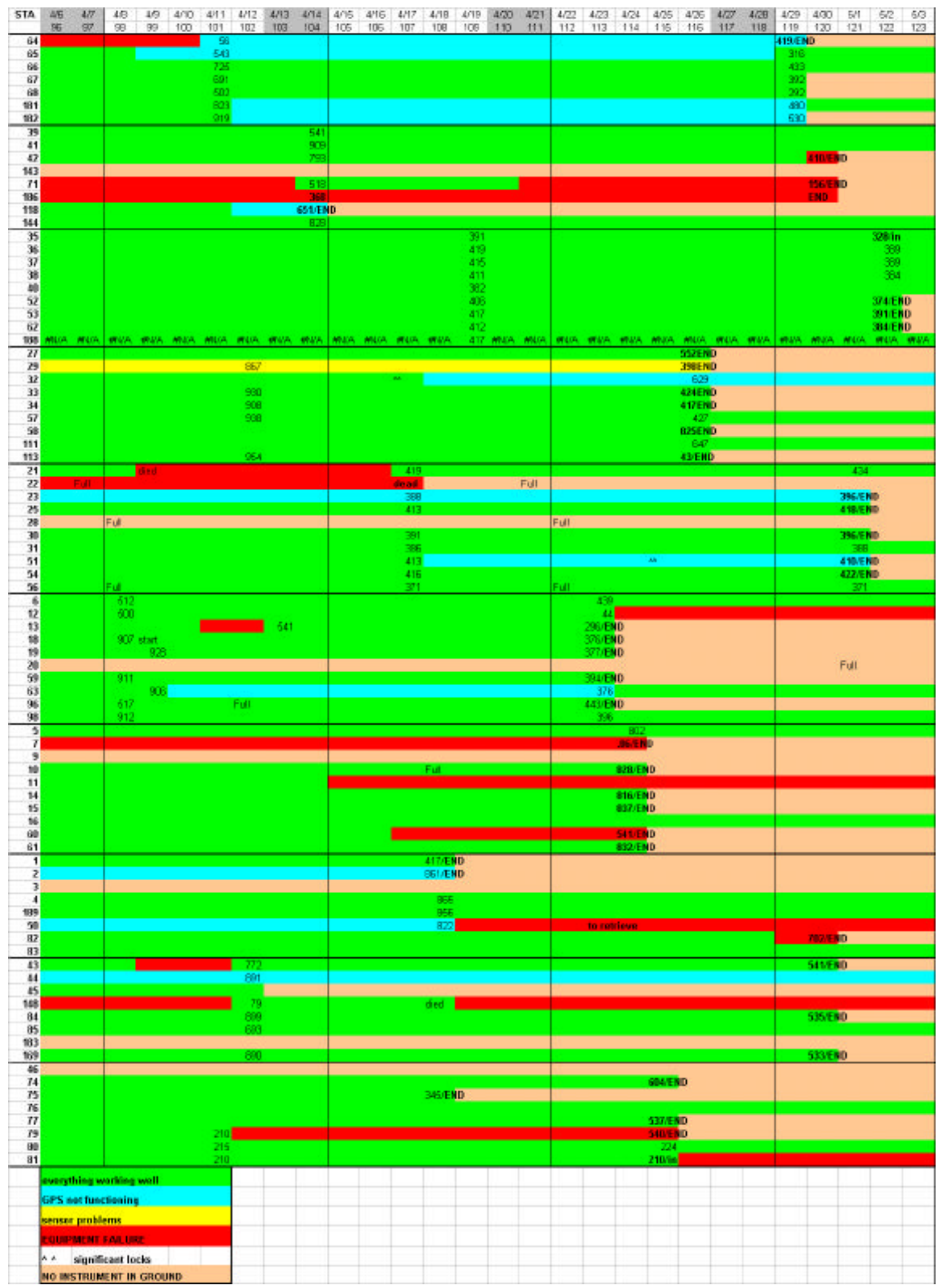




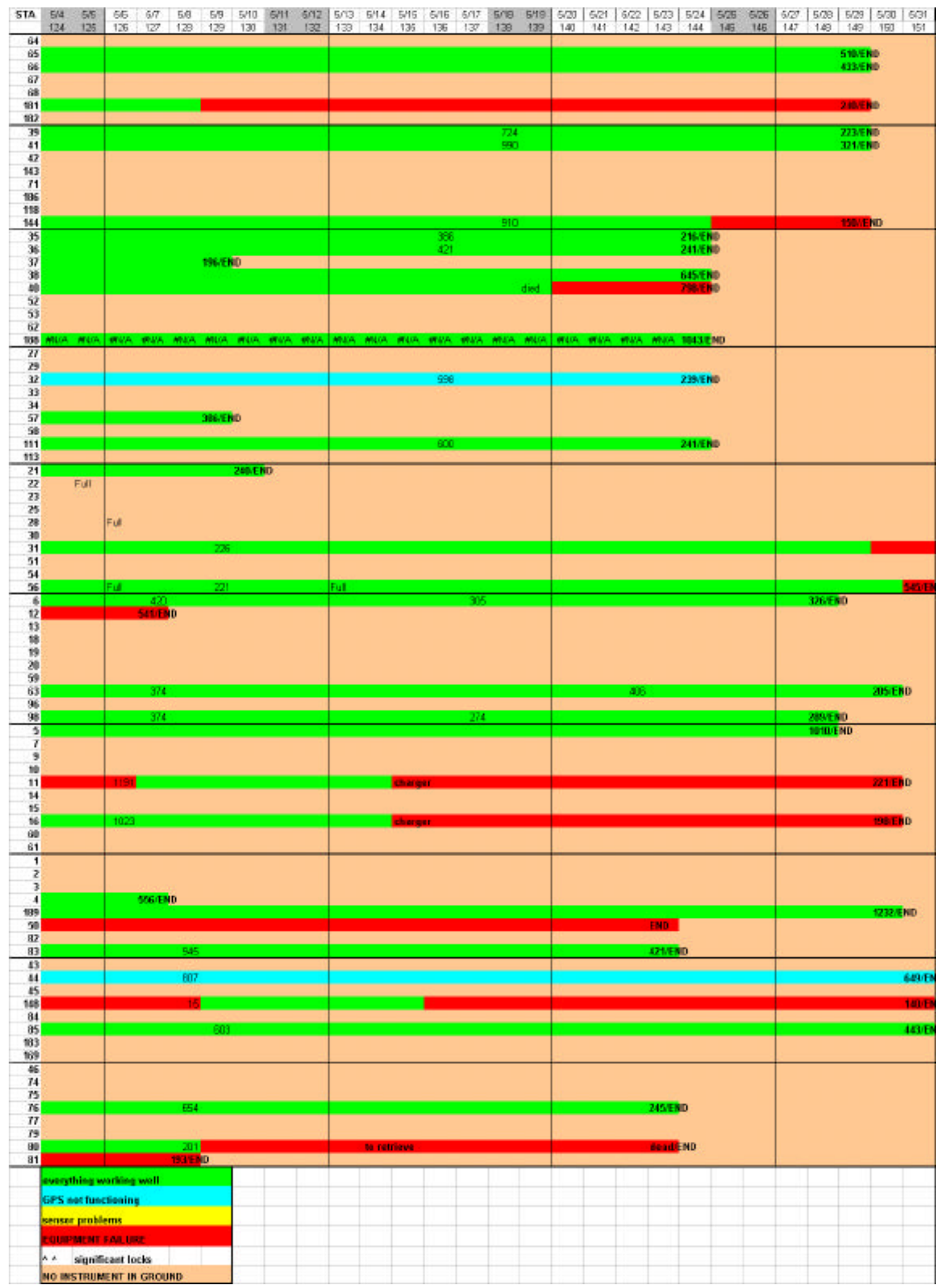




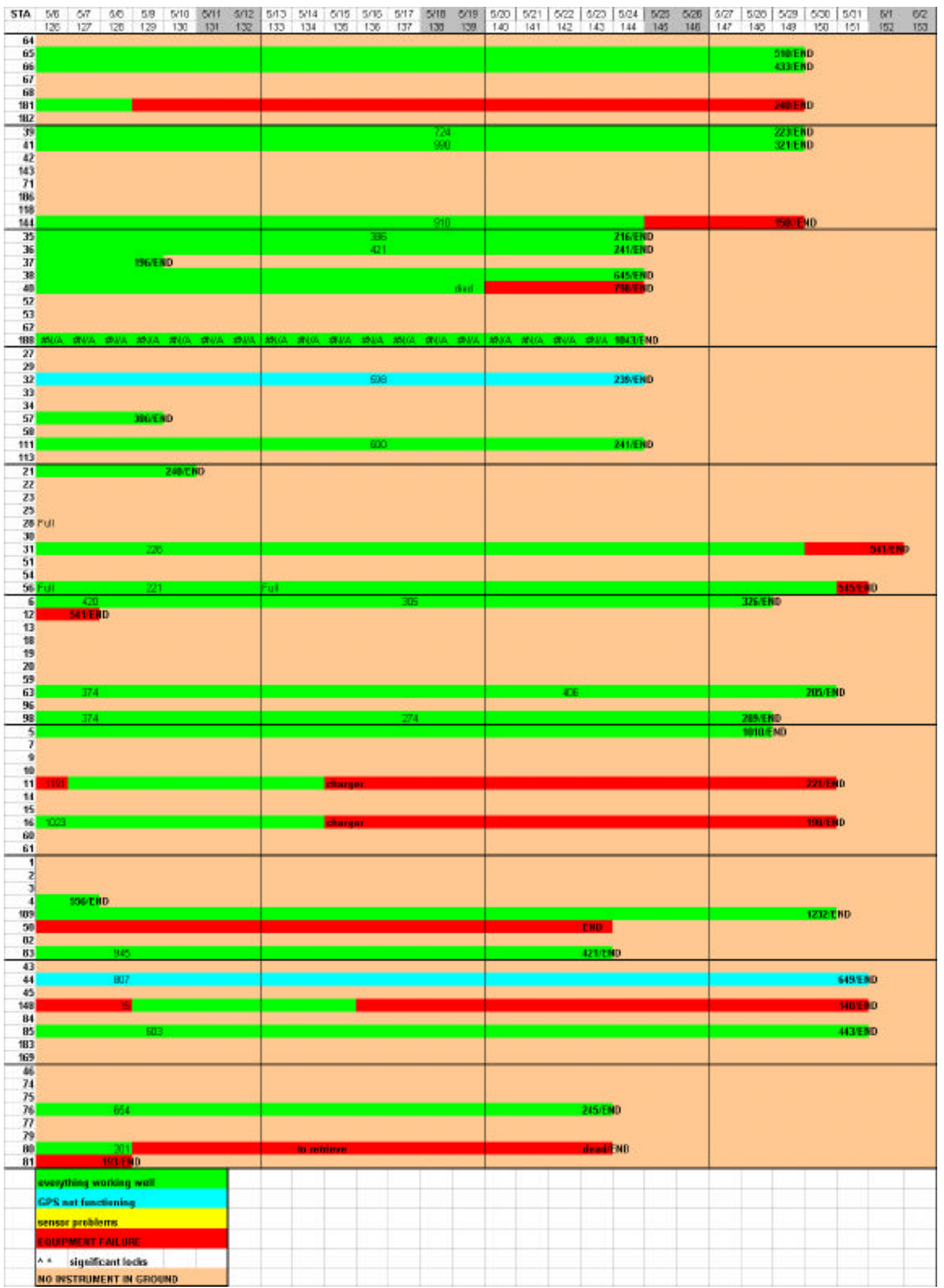

